\title{
Renormalization group evolution of the Standard Model dimension six operators III: gauge coupling dependence and phenomenology
}

\author{
Rodrigo Alonso, ${ }^{a}$ Elizabeth E. Jenkins, ${ }^{a}$ Aneesh V. Manohar ${ }^{a}$ and Michael $\operatorname{Trott}^{b, 1}$ \\ ${ }^{a}$ Department of Physics, University of California at San Diego, \\ 9500 Gilman Drive, La Jolla, CA 92093-0319, U.S.A. \\ b Theory Division, Physics Department, CERN, \\ CH-1211 Geneva 23, Switzerland \\ E-mail: ralonsod@ucsd.edu, ejenkins@ucsd.edu, amanohar@ucsd.edu, \\ michael.trott@cern.ch
}

ABSTRACT: We calculate the gauge terms of the one-loop anomalous dimension matrix for the dimension-six operators of the Standard Model effective field theory (SM EFT). Combining these results with our previous results for the $\lambda$ and Yukawa coupling terms completes the calculation of the one-loop anomalous dimension matrix for the dimensionsix operators. There are $1350 C P$-even and $1149 C P$-odd parameters in the dimensionsix Lagrangian for 3 generations, and our results give the entire $2499 \times 2499$ anomalous dimension matrix. We discuss how the renormalization of the dimension-six operators, and the additional renormalization of the dimension $d \leq 4$ terms of the SM Lagrangian due to dimension-six operators, lays the groundwork for future precision studies of the SM EFT aimed at constraining the effects of new physics through precision measurements at the electroweak scale. As some sample applications, we discuss some aspects of the full RGE improved result for essential processes such as $g g \rightarrow h, h \rightarrow \gamma \gamma$ and $h \rightarrow Z \gamma$, for Higgs couplings to fermions, for the precision electroweak parameters $S$ and $T$, and for the operators that modify important processes in precision electroweak phenomenology, such as the three-body Higgs boson decay $h \rightarrow Z \ell^{+} \ell^{-}$and triple gauge boson couplings. We discuss how the renormalization group improved results can be used to study the flavor problem in the SM EFT, and to test the minimal flavor violation (MFV) hypothesis. We briefly discuss the renormalization effects on the dipole coefficient $C_{e \gamma}$ which contributes to $\mu \rightarrow e \gamma$ and to the muon and electron magnetic and electric dipole moments.

KEYwords: Renormalization Group, Standard Model, Beyond Standard Model

ARXIV EPRINT: 1312.2014

\footnotetext{
${ }^{1}$ Corresponding author.
} 


\section{Contents}

1 Introduction 1

2 The anomalous dimension matrix 3

2.1 The structure of $\gamma_{i j}$

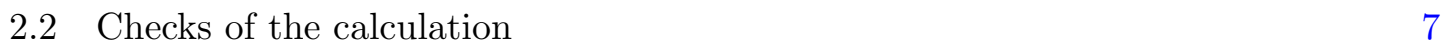

2.3 Cancellations 7

$\begin{array}{lll}2.4 & \text { Previous work } & 10\end{array}$

3 SILH operators $\quad 11$

4 Minimal Flavor Violation 13

5 Phenomenology 14

$\begin{array}{lll}5.1 & \text { Higgs mass and self-couplings } & 15\end{array}$

$\begin{array}{lll}5.2 & \text { Yukawa couplings } & 16\end{array}$

$\begin{array}{lll}5.3 G_{F} & 17\end{array}$

$\begin{array}{ll}5.4 \text { Gauge boson masses and couplings } & 17\end{array}$

$\begin{array}{ll}5.5 & \text { RGE for } C_{H}, C_{H D}, C_{H \square} \\ 5.6 & 19\end{array}$

$\begin{array}{ll}5.6 h \rightarrow f \bar{f} & 20\end{array}$

$5.7 h \rightarrow W W$ and $h \rightarrow Z Z \quad 21$

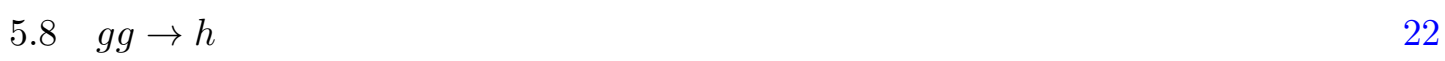

$5.9 h \rightarrow \gamma \gamma \quad 23$

$5.10 h \rightarrow \gamma Z$

5.11 Electroweak precision observables 25

5.12 Triple gauge boson couplings $\quad 25$

$5.13 \mu \rightarrow e \gamma$, magnetic moments, and electric dipole moments 26

$\begin{array}{llr}6 & \text { Conclusions } & 28\end{array}$

$\begin{array}{ll}\text { A Flavor representations and parameter counting } & 28\end{array}$

$\begin{array}{ll}\text { B Conversion of } P_{i} \text { operators to the standard basis } & 31\end{array}$

C Results $\quad 32$

$\begin{array}{lll}\text { C.1 } & X^{3} & 32\end{array}$

$\begin{array}{lll}\text { C. } 2 H^{6} & 32\end{array}$

C.3 $H^{4} D^{2}-32$

C.4 $X^{2} H^{2}$

C.5 $\psi^{2} H^{3}$

C.6 $\psi^{2} X H$

C.7 $\psi^{2} H^{2} D$ 
$\begin{array}{lll}\text { C.8 } & \psi^{4} & 35\end{array}$

C.8.1 $(\bar{L} L)(\bar{L} L) \quad 35$

C.8.2 $(\bar{R} R)(\bar{R} R) \quad 36$

C.8.3 $(\bar{L} L)(\bar{R} R) \quad 38$

C.8.4 $(\bar{L} R)(\bar{R} L) \quad 39$

$\begin{array}{ll}\text { C.8.5 } & (\bar{L} R)(\bar{L} R)\end{array}$

\section{Introduction}

The LHC experiments have recently found strong evidence for a scalar particle with mass $126 \mathrm{GeV}$, and properties consistent with the Higgs boson of the Standard Model (SM) [1, 2]. The absence of any clear evidence of new particles at energies up to several times the scalar boson mass allows one to parametrize the effects of arbitrary new physics residing at energies $\Lambda \gg v$ on physical observables at the electroweak scale in terms of higher dimension operators built out of SM fields. Experimental measurements of the properties of the scalar boson and other observables at the electroweak scale can then be used to constrain or determine the coefficients of the higher dimension operators, and hence the effects of arbitrary beyond-the-standard-model (BSM) theories with characteristic energy scale $\Lambda$ in a model independent way.

In this paper, we adopt the assumption that the scalar boson observed at LHC is the SM Higgs boson, and that the Higgs mechanism generates the mass of the SM gauge fields and fermions. Specifically, we assume that the observed scalar boson $h$ is part of a $\mathrm{SU}(2)_{L}$ doublet $H$ with hypercharge $\mathrm{y}_{H}=\frac{1}{2}$, and that the electroweak $\mathrm{SU}(2)_{L} \times \mathrm{U}(1)_{Y}$ gauge symmetry is a linearly realized symmetry in the scalar sector which is spontaneously broken by the vacuum expectation value of $H$. These assumptions yield the simplest and most direct interpretation of the LHC data, and the related experimental observations from LEP and the Tevatron. ${ }^{1}$ The SM effective field theory (SM EFT) based on these assumptions consists of the SM Lagrangian plus all possible higher dimension operators.

The leading higher dimension operators built out of SM fields that preserve baryon and lepton number are 59 dimension-six operators $[8,9]$. It is important to keep in mind that many of these operators have flavor (generation) indices. For $n_{g}=3$ generations, the dimension-six Lagrangian has $1350 C P$-even and $1149 C P$-odd couplings, for a total of 2499 hermitian operators and real parameters. The flavor indices obviously cannot be neglected - there is no reason in general, for example, why the new physics contribution to $\mu \rightarrow e \gamma$ should be the same as the new physics contribution to the muon magnetic moment. Despite the large number of operators, it is important to realize that the SM equations of motion (EOM) have been used extensively in reducing the operator basis. As a result, the coefficient of a removed operator is distributed among the remaining operators.

\footnotetext{
${ }^{1}$ There are other alternatives being investigated, such as a nonlinearly realized $\mathrm{SU}(2)_{L} \times \mathrm{U}(1)_{Y}$ gauge symmetry in the scalar sector with a light scalar $h$ : see $[3-7]$ and references therein.
} 
In this work, we complete the full calculation of the $2499 \times 2499$ one-loop anomalous dimension matrix of the 59 dimension-six operators in the operator basis of ref. [8, 9], including flavor indices for an arbitrary number of generations $n_{g}$. We present the gauge coupling terms in the one-loop anomalous dimension matrix in this paper. Combined with our past results [10-13], this gives the full one-loop renormalization group evolution (RGE) of the dimension-six operators of the SM EFT. Having the full one-loop RGE of an independent set of dimension-six operators in the SM EFT has the advantage that all physical effects are included, and there can be no cancellation of terms between independent operators.

To precisely interpret any pattern of deviations of SM processes using higher dimensional operators, one has to map the pattern of deviations observed at the electroweak scale back to the scale $\Lambda$, where the BSM physics was integrated out of the effective field theory. Due to operator mixing, the pattern of Wilson coefficients that are observed at the low scale $\sim m_{H}$ is not identical to the pattern of Wilson coefficients at the matching scale $\Lambda$. Our RG calculation determines all of the logarithmically enhanced terms in observables at the renormalization group scale $\mu=m_{H}$ due to RG running from the high-energy scale of new physics $\mu=\Lambda$.

There are also other contributions from the finite parts of one-loop graphs at the low scale $\mu \sim m_{H}$, which we have not computed. For $\Lambda \sim 1 \mathrm{TeV}, \ln \left(\Lambda^{2} / m_{H}^{2}\right) \sim 4$, so there is a modest enhancement of the log terms over the finite terms. As experiments get more precise, and the scale $\Lambda$ is pushed higher, the log terms become even more important relative to the finite terms. Nevertheless, the calculation of finite terms is important, and these terms will eventually be required for a precise comparison of data with the SM EFT. The anomalous dimensions can also be viewed as computing the $\ln \Lambda / m_{H}$ enhanced finite terms. The anomalous dimension computation is easier because it can be done in the unbroken theory, whereas the computation of finite terms needs to be done in the broken theory.

An important application of the SM EFT is to test the hypothesis of minimal flavor violation $[14,15]$. The dimension-six operators can have arbitrary flavor structure, and the renormalization group equations derived in refs. [10-13] and in this paper give non-trivial mixing between different particle sectors. MFV assumes that the only sources of $\mathrm{U}(3)^{5} \mathrm{fla}-$ vor symmetry violation are the Yukawa coupling matrices $Y_{e}, Y_{u}$ and $Y_{d}$. The SM respects MFV by definition. Since MFV is formulated in terms of symmetries, it is preserved by the $\mathrm{RG}$ evolution. If the dimension-six Lagrangian respects MFV, then the RG evolution preserves this property.

The general dimension-six Lagrangian does not have to respect MFV, and RG evolution then feeds non-minimal flavor violation into different operator sectors. By constraining the parameters of the SM EFT, one can experimentally test the MFV hypothesis taking this RG running into account. It is important to test MFV directly in a model-independent way. The SM EFT provides a model-independent formalism to test the MFV hypothesis.

The outline of this paper is as follows. In section 2, we discuss our notation, and the gauge coupling constant terms reported in this work. Some generalities about the structure of the anomalous dimension matrix are given in section 2.1. Some interesting cancellations are pointed out in section 2.3. A detailed presentation of the gauge coupling constant terms in the RG equations of the dimension-six operator coefficients is relegated to appendix C. 
Section 3 compares the standard operator basis of refs. [8, 9] with SILH operators [16]. A brief discussion of MFV and its implications is given in section 4. Section 5 presents the main applications of the SM EFT to phenomenology. We discuss the SM parameters at tree level, and how their values are modified by the SM EFT dimension-six operators. In particular, we discuss the modifications to the Higgs mass and couplings, and to the gauge boson masses. We also discuss the scale dependence of the dimension-six operators, and how the dimension-six operators contribute to the running of the $d \leq 4$ parameters of the SM Lagrangian. The complete expressions for the running of the $g g \rightarrow h, h \rightarrow \gamma \gamma$ and $h \rightarrow \gamma Z$ amplitudes are given in sections 5.8, 5.9, and 5.10, respectively. In sections 5.11 and 5.12, we discuss the operators corresponding to the electroweak precision data (EWPD) parameters $S$ and $T$, and operators modifying critical processes for precision electroweak phenomenology, such as triple gauge boson couplings and the three-body decay $h \rightarrow Z \ell^{+} \ell^{-}$. In section 5.13, we discuss the dipole coefficients $C_{e \gamma}$ which contribute to the decay $\mu \rightarrow e \gamma$ and to the muon and electron magnetic and electric dipole moments. We present our conclusions in section 6 . The counting of parameters in $\mathcal{L}^{(6)}$ is summarized in appendix A, and the conversion of SILH operators to the standard basis is given in appendix B.

\section{The anomalous dimension matrix}

The complete list of 59 independent dimension-six operators is given in table 1 . The operators are divided into eight classes by field content and number of covariant derivatives. The eight operator classes are $1: X^{3}, 2: H^{6}, 3: H^{4} D^{2}, 4: X^{2} H^{2}, 5: \psi^{2} H^{3}, 6: \psi^{2} X H$, $7: \psi^{2} H^{2} D$ and $8: \psi^{4}$, where $X=G_{\mu \nu}^{A}, W_{\mu \nu}^{I}, B_{\mu \nu}$ represents a gauge field strength, $H$ denotes the Higgs doublet scalar field, $\psi$ is a fermion field $\psi=q, u, d, l, e$, and $D$ is a covariant derivative. The dimension-six Lagrangian is

$$
\mathcal{L}^{(6)}=\sum_{i} C_{i} Q_{i}
$$

where the $Q_{i}$ are the dimension-six operators of table 1 and the operator coefficients $C_{i}$ have dimensions of $1 / \Lambda^{2}$. The one-loop anomalous dimension matrix $\gamma_{i j}$ is defined by the $\mathrm{RG}$ equation of the operator coefficients

$$
\dot{C}_{i} \equiv 16 \pi^{2} \mu \frac{\mathrm{d} C_{i}}{\mathrm{~d} \mu}=\gamma_{i j} C_{j} .
$$

The explicit RG equations are given in appendix $\mathrm{C}$ as differential equations, rather than as elements of the matrix $\gamma$. We will use $\gamma_{i j}$ to represent the $8 \times 8$ block form of the anomalous dimension matrix, where the subscripts on $\gamma$ refer to the eight operator classes $i, j=1, \ldots, 8$. For example, $\gamma_{35}$ is the $2 \times 3$ anomalous dimension submatrix which mixes the 3 independent class 5 operator coefficients into the 2 independent class 3 operator coefficients (see table 1 ).

Although there are 59 independent operators, many of them have flavor indices which take on $n_{g}=3$ values. Table 2 gives the number of $C P$-even and $C P$-odd coefficients for each operator class. For $n_{g}=3$, there are $\left(107 n_{g}^{4}+2 n_{g}^{3}+213 n_{g}^{2}+30 n_{g}+72\right) / 8=1350$ 
$C P$-even coefficients and $\left(107 n_{g}^{4}+2 n_{g}^{3}+57 n_{g}^{2}-30 n_{g}+48\right) / 8=1149 C P$-odd parameters, for a total of 2499 parameters which need to be constrained by experiment. The counting of parameters is summarized in appendix A.

Such a large number of terms makes the calculation of the complete anomalous dimension matrix a formidable task. In ref. [13], we began by computing the $8 \times 8$ one-loop anomalous dimension matrix $\gamma_{44}$ for the class-4 Higgs-gauge operators $X^{2} H^{2}$, since these operators contribute directly to the experimentally interesting Higgs production and decay channels $g g \rightarrow h, h \rightarrow \gamma \gamma$, and $h \rightarrow \gamma Z$, which first occur at one loop in the SM. The $8 \times 8$ submatrix $\gamma_{44}$ has been subsequently verified by several independent calculations (e.g. ref. [17]). In ref. [12], we calculated the $\lambda$-dependent terms of the full anomalous dimension matrix for vanishing gauge coupling constants, as well as the complete running of the SM $d \leq 4$ parameters due to the dimension-six operators. The running of the SM parameters resulting from the dimension-six operators is of order $m_{H}^{2} / \Lambda^{2}$, which is of the same order as the tree-level contribution of dimension-six operators. The Yukawa-dependent terms of the anomalous dimension matrix for vanishing gauge couplings were computed in ref. [10]. In this paper, we complete the full calculation of the one-loop anomalous dimension matrix of the dimension-six operators by computing the gauge coupling terms.

The one-loop anomalous dimension matrix has the usual $1 /\left(16 \pi^{2}\right)$ suppression of a oneloop calculation. However, there are several anomalous dimensions with large numerical factors. In ref. [12], for example, we found that

$$
16 \pi^{2} \mu \frac{\mathrm{d}}{\mathrm{d} \mu} C_{H}=108 \lambda C_{H}+\ldots
$$

Since $m_{H}^{2}=2 \lambda v^{2}$, the anomalous dimension coefficient is $108 \lambda=54 m_{H}^{2} / v^{2} \simeq 14$, independent of the normalization convention for the quartic coupling $\lambda$. In the study of the Yukawa coupling terms of ref. [10], the numerical factors were generally $\mathcal{O}(1)$. These Yukawa terms give interesting nontrivial flavor mixing between the various operators. The gauge terms calculated in this paper also contain several large coefficients. For example, the mixing of the class 4 operators $X^{2} H^{2}$ into the class 2 operator $H^{6}$ gives

$$
16 \pi^{2} \mu \frac{\mathrm{d}}{\mathrm{d} \mu} C_{H}=-\left(48 g_{1}^{4} \mathrm{y}_{H}^{4}+12 g_{1}^{2} g_{2}^{2} \mathrm{y}_{H}^{2}\right) C_{H B} \ldots
$$

The lengthiest contributions to gauge coupling constant terms come from the wellknown penguin graph figure 1. The penguin graph itself is simple to compute. However, there are 25 possible $\psi^{4}$ operators in the $\mathcal{L}^{(6)}$ Lagrangian, and the penguin graph is proportional to $D_{\mu} X^{\mu \nu}$, which is replaced by a gauge current summed over all fermion and scalar fields. The resulting four-fermion and fermion-scalar operators then have to be Fierzed to the canonical operator basis, resulting in the bulk of the terms given in appendix C.

One finds a substantial amount of operator mixing in the SM EFT, and such mixing affects observables measured at the electroweak scale in a manner which must be unraveled to understand BSM theories. One of the consequences of this mixing is the propagation of $C P$ violation through different sectors of the Lagrangian. For instance, dipole operators receive contributions from $C P$ violating class 4 operators (that enter, e.g., $h \rightarrow \gamma Z$ 


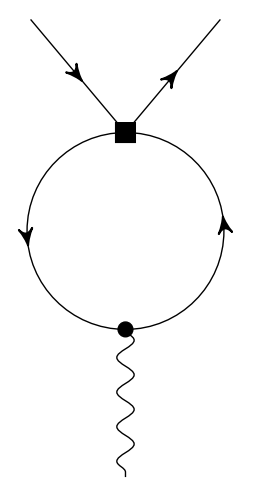

Figure 1. A penguin diagram. The solid square is a $\psi^{4}$ vertex from $\mathcal{L}^{(6)}$, and the dot is a SM gauge coupling.

at tree level), the latter are therefore subject to electric dipole moment constraints, see section 5.13. On the other hand, it is already known [13] that mixing effects are relevant for studies of $h \rightarrow \gamma \gamma$.

\subsection{The structure of $\gamma_{i j}$}

The complication of dealing with a large operator basis naturally leads to the desire to simplify the calculation, or to look for hidden structure in the anomalous dimension matrix to more easily understand the physics of the one-loop RGE flow. In ref. [12], we showed that the structure of the anomalous dimension matrix can be understood using Naive Dimensional Analysis (NDA) [18]. The argument is simplest using rescaled operators $\widehat{Q}_{i}$. The rescaled operators $\widehat{Q}_{i}$ are given by $g^{2} X^{3}, H^{6}, H^{4} D^{2}, g^{2} X^{2} H^{2}, y \psi^{2} H^{3}, g y \psi^{2} X H$, $\psi^{2} H^{2} D$ and $\psi^{4}$, where each gauge field strength $X$ has been rescaled by a gauge coupling $g$, and the chirality-flip operators $\psi^{2} H^{3}$ and $\psi^{2} X H$, which change chirality by one unit, have been rescaled by an additional Yukawa coupling $y$. The dimension-six Lagrangian can be rewritten in terms of the rescaled operators and their corresponding coefficients $\widehat{C}_{i}$,

$$
\mathcal{L}^{(6)}=\sum_{i} C_{i} Q_{i}=\sum_{i} \widehat{C}_{i} \widehat{Q}_{i}
$$

The RG equations for the original and rescaled operator coefficients are given by

$$
\dot{C}_{i}=\gamma_{i j} C_{j}, \quad \dot{\widehat{C}}_{i}=\widehat{\gamma}_{i j} \widehat{C}_{j},
$$

where the one-loop anomalous dimension matrices $\gamma_{i j}$ and $\widehat{\gamma}_{i j}$ are related to each other by the rescaling factors and their derivatives. In ref. [12], we showed that the anomalous dimension matrix $\widehat{\gamma}$ for the rescaled operators has entries proportional to

$$
\widehat{\gamma} \propto\left(\frac{\lambda}{16 \pi^{2}}\right)^{n_{\lambda}}\left(\frac{y^{2}}{16 \pi^{2}}\right)^{n_{y}}\left(\frac{g^{2}}{16 \pi^{2}}\right)^{n_{g}}, \quad \quad N=n_{\lambda}+n_{y}+n_{g}
$$

where $N$, the perturbative order of the anomalous dimension, is defined as the sum of the number of factors $n_{\lambda}$ of the Higgs self-coupling $\lambda$, the number of factors $n_{y}$ of $y^{2}$, and the number of factors $n_{g}$ of $g^{2}$. For the rescaled dimension-six operators, $N$ ranges from 0 to 
4. In ref. [11], we derived a general formula for the perturbative order $N$ of the anomalous dimension matrix $\widehat{\gamma}_{i j}$,

$$
N=1+w_{i}-w_{j}
$$

where $w_{i}$ is the NDA weight of the operators $\widehat{Q}_{i}$ in the $i^{\text {th }}$ class [11]. The class 2 operator $\widehat{Q}_{H}$ has NDA weight $w_{2}=2$; the operators in classes $\{3,5,7,8\}$ have NDA weight 1 ; the operators in classes $\{4,6\}$ have NDA weight 0 ; and the class 1 operators have NDA weight $w_{1}=-1$. Using eq. (2.8), the possible coupling constant dependences of $\widehat{\gamma}_{i j}$ are obtained. Our previous work calculated all anomalous dimensions with nontrivial $n_{\lambda}$ and $n_{y}$ with $n_{g}=0$. The present work completes the calculation of all terms with $n_{g} \neq 0$.

Although the coupling constant dependence of the anomalous dimension matrix is simplest for the NDA rescaled operators, the RGE in refs. $[10,12,13]$ and in this work are quoted in terms of the original unrescaled operators $Q_{i}$ of refs. [8, 9]. The possible entries of $\gamma_{i j}$ were classified in ref. [12] by studying all possible one-loop diagrams including EOM terms. The classification is a bit subtle. The non-zero entries arise directly from diagrams which contribute to a given term, but also indirectly via EOM. For example, the $H^{4} D^{2}-H^{4} D^{2}$ entry of the anomalous dimension matrix is computed from graphs with one insertion of a $H^{4} D^{2}$ operator, $Q_{H \square}$ or $Q_{H D}$, with 4 external $H$ lines. These graphs contribute to the $\gamma_{33}$ submatrix for the running of the coefficients $C_{H \square}$ and $C_{H D}$. The graphs contributing to $\gamma_{33}$ also require a counterterm proportional to the EOM operator $E_{H \square}$ of ref. [12]. This operator can be eliminated in favor of other operators such as the $\psi^{2} H^{3}$ operators in the standard basis. Thus, the $\gamma_{33}$ graphs also contribute to the $\gamma_{53}$ submatrix via the EOM, even though they do not have any external fermion lines.

The NDA weights $w_{i}$ for the NDA rescaled operators $\widehat{Q}_{i}$ of the eight operator classes, and the coupling constant dependence of the allowed anomalous dimensions $\widehat{\gamma}_{i j}$ are shown in table 3 , with the operators ordered according to decreasing NDA weight. Now that the entire matrix has been computed, we can compare with the classification of ref. [12]. The cross-hatched entries in the table are anomalous dimension entries which could exist based on the allowed diagrams, but which vanish by explicit computation. These entries vanish because the relevant diagram vanishes, has no infinite part despite being naively divergent, or, in some interesting cases, by cancellation between different contributions such as a direct contribution to $\gamma_{i j}$ and an indirect contribution obtained by using the EOM. These cancellations are discussed further in section 2.3.

The diagonal blocks in table 3 have $N=1$ since $w_{i}=w_{j}$. Blocks one below the diagonal have $N=0$, whereas blocks one above the diagonal have $N=2$, etc. When $N$ is less than $0, \gamma$ vanishes, and we find that this is always the case. However, there are many additional anomalous dimensions which vanish. Indeed, almost all of the $N=0$ entries vanish. The notable exception of a $N=0$ submatrix which does not vanish is $\gamma_{68}$ which mixes class 8 four-fermion operators $\psi^{4}$ into the class 6 dipole operators $\psi^{2} X H$ in violation of the general "no tree-loop mixing" claim of refs. [19-21]. Other examples which violate no tree-loop mixing exist [22]. "Tree-loop" classification [23] of terms in an EFT Lagrangian has limited usefulness, and does not apply in general when the UV theory generating the dimension-six operators is itself an EFT, or is a strongly interacting theory. 
Attempts to broaden this classification scheme in a very general manner relied critically on the assumption of minimal coupling. However, in ref. [24], we showed that the concept of minimal coupling is ill defined in general.

\subsection{Checks of the calculation}

The calculations in this paper are done in background field with gauge fixing parameter $\xi$, and cancellation of $\xi$-dependence provides a check on the results. The gauge dependence only cancels for gauge-invariant interactions, i.e. if the relations

$$
\mathrm{y}_{q}=\mathrm{y}_{d}+\mathrm{y}_{H}, \quad \mathrm{y}_{q}=\mathrm{y}_{u}-\mathrm{y}_{H}, \quad \mathrm{y}_{l}=\mathrm{y}_{e}+\mathrm{y}_{H},
$$

are satisfied. Although the expressions for the anomalous dimensions have been written in terms of all six hypercharges, $y_{i}$ cannot be thought of as varying independently, but must satisfy the constraints eq. (2.9). A check of the results that follows from custodial $\mathrm{SU}(2)$ symmetry is discussed at the end of section 5.11.

The SM Yukawa couplings

$$
\mathcal{L}_{\text {Yukawa }}=-\left[H^{\dagger j} \bar{d}_{r}\left[Y_{d}\right]_{r s} q_{j s}+\widetilde{H}^{\dagger j} \bar{u}_{r}\left[Y_{u}\right]_{r s} q_{j s}+H^{\dagger j} \bar{e}_{r}\left[Y_{e}\right]_{r s} l_{j s}+\text { h.c. }\right],
$$

where $r, s$ are flavor indices and $j$ is an $\mathrm{SU}(2)$ index, are only gauge invariant because the 2 of $\mathrm{SU}(2)$ is self-conjugate, so that $H_{j}$ and $\widetilde{H}_{j}=\epsilon_{j k} H^{\dagger k}$ belong to the same $\mathrm{SU}(2)$ representation. The $\mathrm{SU}(2)$ group cannot be generalized to a $\mathrm{SU}(N)$ group. While some of the $\mathrm{SU}(2)$ group theory factors have been written as Casimirs such as $c_{A, 2}$ and $c_{F, 2}$, the results are only valid when they take on their $\mathrm{SU}(2)$ values $c_{A, 2}=2$ and $c_{F, 2}=3 / 4$.

The $\mathrm{SU}(3)$ results are written for an $\mathrm{SU}\left(N_{c}\right)$ theory. Anomaly cancellation does not hold for the $\mathrm{SU}\left(N_{c}\right)^{2} \times \mathrm{U}(1)_{Y}$ anomaly for arbitrary $N_{c}$, but the results can still be useful in other contexts for the $\mathrm{SU}\left(N_{c}\right)$ anomalous dimensions. The $\mathrm{SU}(3)$ Fierz identity

$$
T_{\alpha \beta}^{A} T_{\lambda \sigma}^{A}=\frac{1}{2} \delta_{\alpha \sigma} \delta_{\lambda \beta}-\frac{1}{2 N_{c}} \delta_{\alpha \beta} \delta_{\lambda \sigma}
$$

has been used to rearrange color indices and put operators into standard form. This identity is valid for the fundamental representation of $\mathrm{SU}\left(N_{c}\right)$, but is not valid for arbitrary representations. Thus, the quadratic Casimir $c_{F, 3}$ is equivalent to $\left(N_{c}^{2}-1\right) /\left(2 N_{c}\right)$, and the fermions must be in $\mathrm{SU}\left(N_{c}\right)$ fundamental or anti-fundamental representations.

\subsection{Cancellations}

The one-loop anomalous dimension matrix does not contain all possible terms that can arise from the allowed one-loop graphs and the EOM. In a few cases, the entries vanish because the graph has no divergent part. An example from ref. [12] is the $y^{4}$ contribution to $\gamma_{27}$, or $H^{6}-\psi^{2} H^{2} D$ mixing.

There also are a few cases with interesting non-trivial cancellations which arise when different contributions to the same anomalous dimension are added together after using the equations of motion. An example is the contribution of insertions of the $C P$-even operators 

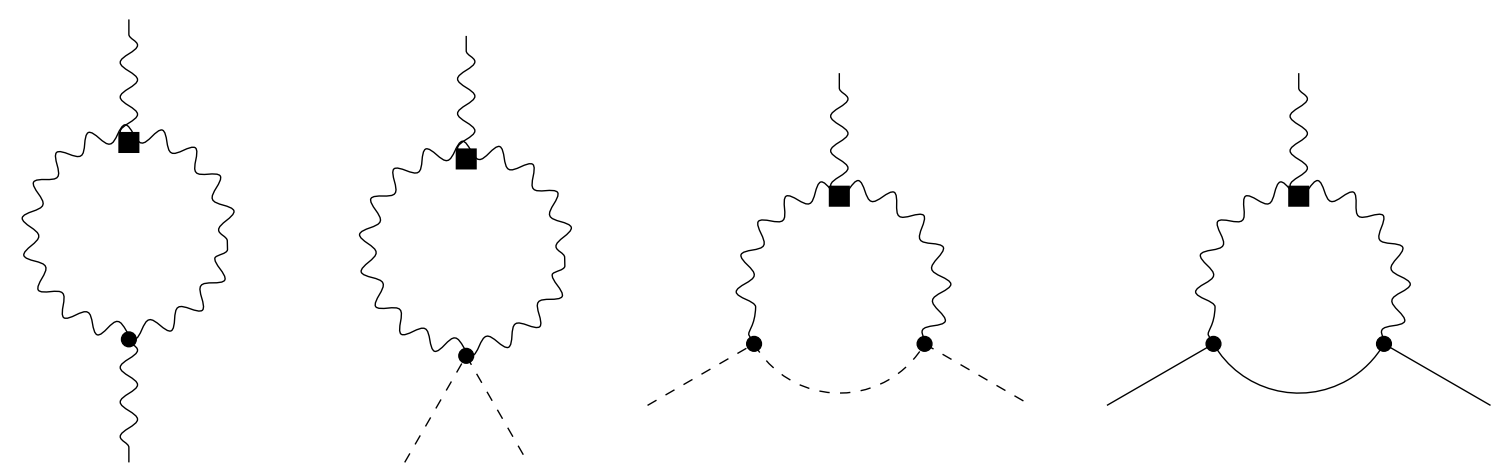

Figure 2. Graphs with insertions of the $X^{3}$ operator which cancel after using the equations of motion.

$X^{3}$ to the anomalous dimension from the graphs shown in figure 2. The divergent part of the first graph is proportional to

$$
A_{1}=-c_{A, 2} g_{2} C_{W} D^{\mu} W_{\mu \lambda}^{I} D_{\nu} W^{I \nu \lambda}-c_{A, 3} g_{3} C_{G} D^{\mu} G_{\mu \lambda}^{A} D_{\nu} G^{A \nu \lambda} .
$$

The divergent part of the sum of the second and third graphs is proportional to

$$
A_{2}=-i g_{2}^{2} c_{A, 2} C_{W} D_{\mu} H^{\dagger} \tau^{I} D_{\nu} H W_{\mu \nu}^{I}
$$

There is no gluon term, since gluons do not couple to the Higgs field. The divergent part of the fourth graph is proportional to

$$
A_{3}=g_{2}^{2} c_{A, 2} C_{W} D^{\mu} W_{\mu \nu}^{I} j_{\psi}^{I \nu}+g_{3}^{2} c_{A, 3} C_{G} D^{\mu} G_{\mu \nu}^{A} j_{\psi}^{A \nu},
$$

where

$$
j_{\psi}^{I \mu}=\sum_{\psi=q, l} \bar{\psi} \gamma^{\mu} \frac{1}{2} \tau^{I} \psi, \quad \quad j_{\psi}^{A \mu}=\sum_{\psi=q, u, d} \bar{\psi} \gamma^{\mu} T^{A} \psi,
$$

are the $\mathrm{SU}(2)$ and $\mathrm{SU}(3)$ fermion currents, respectively. The operator eq. (2.13) is equal to $g_{2} c_{A, 2} C_{W} P_{H W}$, where $P_{H W}$ is given in eq. (3.3). Integrating by parts, and writing the commutator of two covariant derivatives as a field-strength tensor gives the identity

$$
\begin{aligned}
P_{H W} & =-i g_{2} D_{\mu} H^{\dagger} \tau^{I} D_{\nu} H W^{I \mu \nu} \\
& =g_{2} j_{H}^{I \nu} D^{\mu} W_{\mu \nu}^{I}-\frac{1}{4} g_{2}^{2} H^{\dagger} H W_{\mu \nu}^{I} W^{I \mu \nu}-\frac{1}{2} g_{1} g_{2} \mathrm{y}_{H} H^{\dagger} \tau^{I} H W_{\mu \nu}^{I} B_{\mu \nu},
\end{aligned}
$$

where

$$
j_{H}^{I \mu}=\frac{i}{2}\left(H^{\dagger} \tau^{I} \overleftrightarrow{D}^{\mu} H\right)
$$

is the Higgs doublet $\mathrm{SU}(2)$ current. The total is

$$
\begin{aligned}
A_{1}+A_{2}+A_{3}= & -g_{2} c_{A, 2} C_{W} D^{\mu} W_{\mu \lambda}^{I}\left[D_{\nu} W^{I \nu \lambda}-g_{2} j_{\psi}^{I \lambda}-g_{2} j_{H}^{I \lambda}\right] \\
& -c_{A, 3} g_{3} C_{G} D^{\mu} G_{\mu \lambda}^{A}\left[D_{\nu} G^{A \nu \lambda}-g_{2} j_{\psi}^{A \lambda}\right]
\end{aligned}
$$




$$
-g_{2} c_{A, 2} C_{W}\left[\frac{1}{4} g_{2}^{2} H^{\dagger} H W_{\mu \nu}^{I} W^{I \mu \nu}+\frac{1}{2} g_{1} g_{2} \mathrm{y}_{H} H^{\dagger} \tau^{I} H W_{\mu \nu}^{I} B_{\mu \nu}\right]
$$

Using the gauge field equations of motion

$$
D_{\mu} W^{I \mu \nu}=g_{2}\left(j_{H}^{I \mu}+j_{\psi}^{I \mu}\right), \quad D_{\mu} G^{A \mu \nu}=g_{3} j_{\psi}^{A \mu}
$$

only the second line survives,

$$
A_{1}+A_{2}+A_{3}=-g_{2} c_{A, 2} C_{W}\left[\frac{1}{4} g_{2}^{2} H^{\dagger} H W_{\mu \nu}^{I} W^{I \mu \nu}+\frac{1}{2} g_{1} g_{2} \mathrm{y}_{H} H^{\dagger} \tau^{I} H W_{\mu \nu}^{I} B_{\mu \nu}\right] .
$$

The gluon term $C_{G}$ cancels completely and most of the $C_{W}$ term cancels. There is a residual contribution from eq. (2.20) to the anomalous dimension of $C_{H W}$ and $C_{H W B}$, the coefficients of the $X^{2} H^{2}$ Higgs-gauge boson operators. The graphs in figure 2 contribute to the running of $C_{H W}$ and $C_{H W B}$ even though none of the diagrams have two external gauge bosons and two external Higgs lines, the field content of $X^{2} H^{2}$ operators. The cancellation of $C_{G}$ and $C_{W}$ terms in various anomalous dimensions is the reason for the absence of several terms in the last column of table 3.

The $C_{\widetilde{W}}$ and $C_{\widetilde{G}}$ contributions to the anomalous dimension arise from the same graphs as in figure 2, with the insertions of the $C P$-odd operators $\widetilde{X} X X$. In this case, one obtains eqs. (2.12) and (2.14) with $D^{\mu} W_{\mu \nu}^{I}$ and $D^{\mu} G_{\mu \nu}^{A}$ replaced by $D^{\mu} \widetilde{W}_{\mu \nu}^{I}$ and $D^{\mu} \widetilde{G}_{\mu \nu}^{A}$, respectively, and Eq. (2.13) with $W_{\mu \nu}^{I}$ replaced by $\widetilde{W}_{\mu \nu}^{I}$. The equations of motion for $\widetilde{X}$ are $D^{\mu} \widetilde{X}_{\mu \nu}=0$, rather than eq. (2.19), so naively there can be a difference between the $C_{\widetilde{W}, \widetilde{G}}$ and $C_{W, G}$ contributions to the anomalous dimension. However, the total sum $A_{1}+A_{2}+A_{3}$ is

$$
\begin{aligned}
& -g_{2} c_{A, 2} C_{\widetilde{W}} D^{\mu} \widetilde{W}_{\mu \lambda}^{I}\left[D_{\nu} W^{I \nu \lambda}-g_{2} j_{\psi}^{I \lambda}-g_{2} j_{H}^{I \lambda}\right]-c_{A, 3} g_{3} C_{\widetilde{G}} D^{\mu} \widetilde{G}_{\mu \lambda}^{A}\left[D_{\nu} G^{A \nu \lambda}-g_{3} j_{\psi}^{A \lambda}\right] \\
& -g_{2} c_{A, 2} C_{\widetilde{W}}\left[\frac{1}{4} g_{2}^{2} H^{\dagger} H \widetilde{W}_{\mu \nu}^{I} W^{I \mu \nu}+\frac{1}{2} g_{1} g_{2} \mathrm{y}_{H} H^{\dagger} \tau^{I} H \widetilde{W}_{\mu \nu}^{I} B_{\mu \nu}\right]
\end{aligned}
$$

instead of eq. (2.18). The first lines in both eq. (2.18) and eq. (2.21), which would have produced a difference in the $C_{\widetilde{W}, \widetilde{G}}$ and $C_{W, G}$ contributions, are proportional to the gauge field equations of motion (2.19) and vanish. Thus, the contributions to the anomalous dimension from the $C P$-odd coefficients $C_{\widetilde{W}, \widetilde{G}}$ are the same as the contributions from the $C P$-even coefficients $C_{W, G}$.

Another interesting cancellation occurs in the contribution of the $\psi^{2} X H$ dipole operators. The coefficients $C_{e W}$, etc. of these operators will be denoted generically by $C_{\psi X}$, where $\psi=e, u, d$. The dipole operators contribute to the running of $\psi^{2} H^{3}$ coefficients $C_{\psi H}$, such as $C_{e H}$, and to the running of $\psi^{2} H^{2} D$ coefficients $C_{H \psi}$, such as $C_{H e}$. The anomalous dimension for the running of $C_{\psi H}$ gets multiple contributions from $C_{\psi X}$ and $C_{\psi X}^{*}$ which arise from graphs with insertions of the $\psi^{2} X H$ dipole operators and their hermitian conjugates. As above, the multiple contributions arise from using the EOM to bring all divergences to the canonical basis. The total contribution of $C_{\psi X}^{*}$ to the running of $C_{\psi H}$ cancels after using the hypercharge constraints eq. (2.9), even though individual contributions do not vanish. The contribution of $C_{\psi X}$ to the running of $C_{\psi H}$ does not cancel. The total contribution of both $C_{\psi X}$ and $C_{\psi X}^{*}$ to the running of the $\psi^{2} H^{2} D$ coefficients $C_{H \psi}$ 
exactly cancels, which is why there is no $g^{2} y^{2}$ entry in the anomalous dimension $\gamma_{76}$ from $\psi^{2} H^{2} D-\psi^{2} X H$ mixing in table 3 .

The contributions of the dipole operators and the gauge operators with $X$ and $\tilde{X}$ are related by factors of $i$. This simple factor follows from the complex self-duality of $\sigma^{\mu \nu} P_{R}$. There is no $C_{\psi X}^{*}$ contribution to the running $\dot{C}_{\psi X}$, or to the runnings $\dot{C}_{q u q d}^{(1)}, \dot{C}_{q u q d}^{(8)}$ and $\dot{C}_{\text {lequ }}^{(3)}$, which are the $\psi^{4}$ operators to which the dipole operators contribute.

The examples above indicate that the RG contribution of the dipole operators respects holomorphy in $C_{\psi X}$.

\subsection{Previous work}

Several of the gauge coupling terms of the one-loop anomalous dimension matrix have been calculated before. However, we emphasize that with the results reported in this work, we have determined the complete one-loop anomalous dimension matrix for dimension-six operators of the SM EFT for the first time.

Previous calculations of individual elements of the anomalous dimension matrix include the following works. ${ }^{2}$ The anomalous dimension of $Q_{G}$ and $Q_{\tilde{G}}$ were determined in refs. [25-27]. We agree with this result. Ref. [25] computed the anomalous dimension of dimension-five and dimension-six operators in QCD. Parts of our calculation in which the Higgs field can be treated as an external constant field agree with these results. The renormalization of four-fermion operators has been studied for many years in the context of the low-energy theory of weak interactions, and provides a check on the $\psi^{4}-\psi^{4}$ anomalous dimension. The complete one-loop RGE of the operators in class 4 was calculated for the first time in ref. [13]. Previously, some individual terms in this running result were calculated in refs. [28-31], and these terms are consistent with our calculation. Ref. [19] calculated the mixing of dipole operators $Q_{u G}, Q_{u W}$ and $Q_{u B}$ with the combination of Wilson coefficients $Q_{H W}, Q_{H B}$ and $Q_{H W B}$ that corresponds to $h \rightarrow \gamma \gamma$, see section 5.9, which corresponds to a set of entries in $\gamma_{46}$. We agree with these results. Ref. [32] reports the running of the operators $Q_{u H}$ and $Q_{u G}$ due to the QCD coupling, which corresponds to entries in $\gamma_{55}$ and $\gamma_{66}$. We agree with the diagonal running results of this paper.

The papers mentioned in the previous paragraph allow a relatively direct comparison between results computed in the same operator basis. Many other results in the literature are reported in a different basis, making a comparison difficult. Ref. [20] presents a few terms in the anomalous dimension matrix without flavor indices (i.e. for $n_{g}=1$ ), and only including the top Yukawa coupling. The exact translation between such partial results and this work requires that a complete non-redundant operator basis be defined, which often is not the case. Ref. [20] does not define such a mapping to allow us to compare our results to the terms reported, see the next section for more discussion on this point. Nevertheless, some other classic past results in refs. [33-46] overlap with some of the results presented here, as do some more recent works [47-54].

\footnotetext{
${ }^{2}$ Due to the number of operators renormalized, and the fragmentary literature on the subject, we apologize in advance to authors whose works are overlooked in this discussion.
} 


\section{SILH operators}

A minimal basis of dimension-six operators is obtained by removing all redundant operators using the SM EOM. This paper uses the dimension-six operators $Q_{i}$ of ref. [9] which has no redundancies. It is a well-established result in quantum field theory that operators which vanish by the classical equations of motion do not contribute to $S$-matrix elements even at the quantum level [55], and so EOM can be used to simplify the effective Lagrangian. Formally, the redundant operators can be eliminated by a change of variables in the functional integral. It is clearly a nuisance to use a redundant operator basis.

Including redundant operators introduces extra parameters in the Lagrangian which can be eliminated by field redefintions, and do not contribute to any measurable quantity [55]. This redundancy is not always obvious, since intermediate steps and partial results can depend on the redundant parameters. It is only when the complete $S$-matrix element is carefully computed that one sees that certain combinations of parameters drop out due to the EOM. Redundant operators have led to enormous confusion in the literature over many decades, for example, this was a source of significant confusion in the early days of heavy quark effective theory. For this reason, when choosing a basis, it is advantageous to not introduce redundant parameters.

Recently, some authors $[20,56,57]$ have advocated using the "SILH-basis." The definition of this operator basis varies in the papers, and the original SILH paper [16] does not define a complete basis. We will discuss the version presented in ref. [56]. The basis of refs. $[8,9]$ contains nine $C P$-even operators made out of only gauge and Higgs fields,

$$
Q_{G}, Q_{W}, Q_{H}, Q_{H \square}, Q_{H D}, Q_{H G}, Q_{H W}, Q_{H B}, Q_{H W B}
$$

The SILH basis defined in ref. [56] contains $14 C P$-even operators made out of only gauge and Higgs fields with the operator coefficients

$$
\bar{c}_{H}, \bar{c}_{T}, \bar{c}_{6}, \bar{c}_{W}, \bar{c}_{B}, \bar{c}_{H W}, \bar{c}_{H B}, \bar{c}_{\gamma}, \bar{c}_{g}, \bar{c}_{3 W}, \bar{c}_{3 G}, \bar{c}_{2 W}, \bar{c}_{2 B}, \bar{c}_{2 G}
$$

The six operators $Q_{G}, Q_{W}, Q_{H}, Q_{H \square}, Q_{H G}, Q_{H B}$ coincide with the operators corresponding to $\bar{c}_{3 G}, \bar{c}_{3 W}, \bar{c}_{6}, \bar{c}_{H}, \bar{c}_{g}, \bar{c}_{\gamma}$, up to simple rescalings by couplings. In ref. [56], it is argued that the three operators corresponding to $\bar{c}_{2 W}, \bar{c}_{2 B}$ and $\bar{c}_{2 G}$ can be removed by the SM EOM in favor of other operators retained in the SILH operator basis. This removal leaves five flavor-singlet operators ${ }^{3}$

$$
\begin{array}{rlrl}
\mathcal{P}_{H W} & =-i g_{2}\left(D^{\mu} H\right)^{\dagger} \tau^{I}\left(D^{\nu} H\right) W_{\mu \nu}^{I}, & \mathcal{P}_{H B} & =-i g_{1}\left(D^{\mu} H\right)^{\dagger}\left(D^{\nu} H\right) B_{\mu \nu} \\
\mathcal{P}_{W} & =-\frac{i g_{2}}{2}\left(H^{\dagger} \tau^{I} \overleftrightarrow{D}^{\mu} H\right)\left(D^{\nu} W_{\mu \nu}^{I}\right), & \mathcal{P}_{B}=-\frac{i g_{1}}{2}\left(H^{\dagger} \overleftrightarrow{D}^{\mu} H\right)\left(D^{\nu} B_{\mu \nu}\right) \\
\mathcal{P}_{T} & =\left(H^{\dagger} \overleftrightarrow{D^{\mu}} H\right)\left(H^{\dagger} \overleftrightarrow{D}^{\mu} H\right), &
\end{array}
$$

in the SILH basis, instead of the three operators

$$
Q_{H W}=H^{\dagger} H W_{\mu \nu}^{I} W_{I}^{\mu \nu}, \quad Q_{H W B}=H^{\dagger} \tau_{I} H W_{\mu \nu}^{I} B^{\mu \nu}, \quad Q_{H D}=\left(H^{\dagger} D^{\mu} H\right)^{\star}\left(H^{\dagger} D_{\mu} H\right),
$$

in the standard basis.

\footnotetext{
${ }^{3}$ The SILH basis operators are denoted by $P_{i}$ to avoid confusion with similarly labelled operators $Q_{i}$ in the standard basis.
} 
Since eq. (3.3) has five operators, and eq. (3.4) has only three operators, two additional operators from the standard $Q_{i}$ basis can be eliminated if the operators in eq. (3.3) are used instead of those in eq. (3.4). The five $P_{i}$ operators can be written in terms of the standard basis $Q_{i}$ using the equations of motion, and the conversion is given in appendix B. The relations involve non-bosonic $Q_{i}$ operators, a fact that is used in ref. [56] to remove the lepton-Higgs operators $Q_{H l}^{(1)}$ and $Q_{H l}^{(3)}$ together with $Q_{H W}, Q_{H W B}$ and $Q_{H D}$ in favor of the $5 P_{i}$ operators of eq. (3.3). However, only the flavor-singlet combinations

$$
\underset{p p}{Q_{H l}^{(1)},}, \quad \underset{p p}{(3)},
$$

enter the relations in eq. (B.1). One can modify the singlet part of the coefficients of $Q_{H l}^{(1)}$ and $Q_{H l}^{(3)}$ by the shift

$$
\underset{r s}{C_{H l}^{(1,3)}} \rightarrow \underset{r s}{C_{H l}^{(1,3)}}+a^{(1,3)} \delta_{r s}
$$

and absorb the change in the $P_{i}$ operator coefficients. The constants $a^{(1,3)}$ can be chosen to eliminate the trace eq. (3.5), or to set the electron operator $\underset{e e}{C_{H l}^{(1)}}=0$, etc. However, the coefficients of the flavor non-singlet parts

$$
C_{r s}^{(1)}-\frac{1}{n_{g}} \delta_{r s} C_{p p}^{(1)}, \quad \underset{r s}{C_{H l}^{(3)}}-\frac{1}{n_{g}} \delta_{r s} C_{p p}^{(3)}
$$

cannot be removed, and must be retained. Removal of the flavor-singlet portions of $C_{H l}^{(1)}$ and $C_{H l}^{(3)}$ makes the treatment of BSM flavor violation in the SILH basis cumbersome. Furthermore, a careful and consistent treatment of EOM effects is necessary in all calculations using the "SILH-basis," otherwise the basis remains redundant.

The lepton-Higgs operators $Q_{H l}^{(1)}$ and $Q_{H l}^{(3)}$ can be removed completely if one assumes completely unbroken $\mathrm{U}(3)^{5}$ flavor symmetry of the UV theory, so that the coefficients of these operators are unit matrices in flavor space. This assumption was explicitly stated in the initial work of ref. [58] that identified this field redefintion, and it is also adopted in refs. $[20,56,57]$. This assumption is stronger than assuming MFV, which only says that the coefficients of the lepton operators is a function of $Y_{e}^{\dagger} Y_{e}$, not that it is proportional to the unit matrix. Ref. [20] computes a few of the anomalous dimensions in the case of a $\mathrm{U}(3)^{5}$ flavor-symmetric BSM sector, in an attempt to circumvent this difficulty. While the assumption of flavor-symmetric BSM physics can be adopted, it limits the applicability of the EFT. One of the important features of the SM EFT is that it can be used to test MFV, but this is only possible if MFV is not put in by hand. Many SILH basis results cannot be used to test MFV in a straightforward manner, since stronger assumptions than MFV have already been built into the formalism.

In reducing the SILH operators to the operator basis of ref. [9], the EOM relations in appendix B also include the SM dimension-four operator $\left(H^{\dagger} H\right)^{2}$, which is the usual $\lambda\left(H^{\dagger} H\right)^{2}$ Higgs interaction term. This means that the connection of the two bases also involves the redefinition of SM parameters. Explicitly, the RGE for the SM parameters also 
have contributions from dimension-six operators, as pointed out in ref. [12]. These effects are not taken into account in ref. [20] preventing a comparison of our results with ref. [20]. ${ }^{4}$

Also note that ref. [20] advocates retaining redundant operators in intermediate steps of the analysis. Retaining redundant operators in partial results for an anomalous dimension matrix introduces spurious gauge and scheme dependence, see the discussion in ref. [12]. It is not defined in ref. [20] how the partial results for the anomalous dimension matrix presented there can be converted to the full results valid for any BSM flavour structure. This is another reason we cannot compare our results with the partial calculation in ref. [20].

\section{Minimal Flavor Violation}

The SM EFT provides a way to test the hypothesis of MFV in new physics. The SM has a $\mathrm{U}(3)^{5}$ symmetry in the limit of vanishing Yukawa couplings under which

$$
q \rightarrow U_{q} q, \quad l \rightarrow U_{l} l, \quad u \rightarrow U_{u} u, \quad d \rightarrow U_{d} d, \quad e \rightarrow U_{e} e .
$$

The MFV hypothesis $[14,15]$ is that the only source of flavor violation is the Yukawa matrices, so that the full theory is flavor invariant if the Yukawa matrices transform as

$$
Y_{u} \rightarrow U_{u} Y_{u} U_{q}^{\dagger}, \quad Y_{d} \rightarrow U_{d} Y_{d} U_{q}^{\dagger}, \quad Y_{e} \rightarrow U_{e} Y_{e} U_{l}^{\dagger} .
$$

If the new physics respects MFV, then the SM EFT derived from it also does. This assumption severely restricts the dimension-six coefficients. The coefficients of the flavor invariant operators in classes 1-4 can only depend on the flavor invariants ${ }^{5}$

$$
\operatorname{Tr} f\left(Y_{e}^{\dagger} Y_{e}\right), \quad \operatorname{Tr} f\left(Y_{d}^{\dagger} Y_{d}, Y_{u}^{\dagger} Y_{u}\right)
$$

In an EFT setup, the dependence on such invariants can be absorbed into an effective coefficient.

The $\psi^{2} H^{3}$ operators have coefficients

$$
C_{d s}=\left[f\left(Y_{d}^{\dagger} Y_{d}, Y_{u}^{\dagger} Y_{u}\right) Y_{d}^{\dagger}\right]_{r s}, \quad C_{r s}=\left[f\left(Y_{d}^{\dagger} Y_{d}, Y_{u}^{\dagger} Y_{u}\right) Y_{u}^{\dagger}\right]_{r s}, \quad C_{r s}=\left[f\left(Y_{e}^{\dagger} Y_{e}\right) Y_{e}^{\dagger}\right]_{r s},
$$

where it is implicit that the above functions also can depend on the invariants of eq. (4.3). For example, the quark functions can depend on the lepton invariant $\operatorname{Tr} f\left(Y_{e}^{\dagger} Y_{e}\right)$ and viceversa. Analogous formulae to eq. (4.4) hold for the $\psi^{2} X H$ dipole operators $\left\{C_{e W}, C_{e B}\right\}$, $\left\{C_{u G}, C_{u W}, C_{u B}\right\}$ and $\left\{C_{d G}, C_{d W}, C_{d B}\right\}$, respectively.

The $\psi^{2} H^{2} D$ operators have coefficients

$$
C_{r s}^{(1,3)}=\left[f\left(Y_{d}^{\dagger} Y_{d}, Y_{u}^{\dagger} Y_{u}\right)\right]_{r s}, \quad \quad C_{r s}^{(1,3)}=\left[f\left(Y_{e}^{\dagger} Y_{e}\right)\right]_{r s},
$$

\footnotetext{
${ }^{4}$ For an example of this effect, see section 5.5, eq. (5.34).

${ }^{5}$ In this section, $f$ denotes an arbitrary function, and all the $f \mathrm{~s}$ do not have to be the same. Some $\mathrm{U}(1) \mathrm{s}$ are anomalous, and one also can have dependence on certain combinations of $\operatorname{det} Y_{u, d, e}$ and the $\theta$ angles [59-61].
} 


$$
\begin{aligned}
C_{r s}^{H u} & =a \delta_{r s}+\left[Y_{u} f\left(Y_{d}^{\dagger} Y_{d}, Y_{u}^{\dagger} Y_{u}\right) Y_{u}^{\dagger}\right]_{r s}, & C_{r s} & =a \delta_{r s}+\left[Y_{d} f\left(Y_{d}^{\dagger} Y_{d}, Y_{u}^{\dagger} Y_{u}\right) Y_{d}^{\dagger}\right]_{r s}, \\
C_{r e} & =a \delta_{r s}+\left[Y_{e} f\left(Y_{e}^{\dagger} Y_{e}\right) Y_{e}^{\dagger}\right]_{r s}, & C_{r u d} & =\left[Y_{u} f\left(Y_{d}^{\dagger} Y_{d}, Y_{u}^{\dagger} Y_{u}\right) Y_{d}^{\dagger}\right]_{r s} .
\end{aligned}
$$

Again, dependence of the above functions of the invariants of eq. (4.3) is implicit.

Similar expressions hold for the $\psi^{4}$ operators, with coefficients in flavor space which are products of the cases considered above. As is well-known, one can make $\mathrm{U}(3)^{5}$ rotations to bring the Yukawa matrices into the form

$$
Y_{e} \rightarrow \operatorname{diag}\left(m_{e}, m_{\mu}, m_{\tau}\right), \quad Y_{d} \rightarrow \operatorname{diag}\left(m_{d}, m_{s}, m_{b}\right), \quad Y_{u} \rightarrow \operatorname{diag}\left(m_{u}, m_{c}, m_{t}\right) K
$$

where $K$ is the CKM matrix. At this stage, the masslessness of neutrinos allows for the diagonalization of $Y_{e}$ and the absence of flavor violation in the lepton sector. The introduction of neutrino masses can be accomplished in the model-independent spirit of this paper via the $d=5$ Weinberg Operator. This operator is naturally suppressed by a scale higher than $\Lambda$ since it violates lepton number. Assuming this hierarchy of scales, the RGEs of $d=5$ and $d=6$ operators are independent and the inclusion of neutrino masses is orthogonal and does not affect the results presented here.

Since MFV is implemented as a symmetry which is respected by the SM Lagrangian, the RG evolution of $\mathcal{L}^{(6)}$ maintains MFV if the coefficients at scale $\Lambda$ satisfy the MFV hypothesis. In this case, the flavor structure of $\mathcal{L}^{(6)}$ is the same as corresponding amplitudes computed from loop graphs in the SM. However, it is important to emphasize that the assumption of MFV does not imply that the coefficients of $\psi^{2} H^{2} D$ and $\psi^{4}$ operators are proportional to the unit matrix, which is a stronger assumption that requires that the functions $f$ have a perturbative expansion in $Y$ with small coefficients. In view of eq. (4.6), this expansion in powers of Yukawa matrices can be justified for off-diagonal elements inducing flavor violation, as customary, but not for the diagonal entry of the third generation, see ref. [62] for some discussion on this point.

One of the important applications of the SM EFT is to test the hypothesis of MFV in BSM physics in a model-independent way. Interestingly, the full SM RGE transfers flavor violation in one set of operators to other operator sectors. Testing the consistency of MFV in low-energy measurements, taking into account the full SM EFT, is important for increasing our understanding of the flavor structure of new physics. A quick look at the anomalous dimensions in refs. $[10,12]$ and appendix $\mathrm{C}$ should convince the reader that any flavor ansatz not based on a symmetry will not be preserved by the RGE.

\section{Phenomenology}

In this section, we outline the generalization of the analysis of observables measured at the electroweak scale from the SM to the SM EFT, and how the full one-loop RGE for the dimension-six Wilson coefficients measured at a low scale $\sim v$ can be used to obtain the Wilson coefficients at the high scale $\Lambda$. An important point we emphasize is that if constraints at the scale $v$ are to be mapped to a high scale BSM theory, then all corrections 
of the order $v^{2} /\left(16 \pi^{2} \Lambda^{2}\right)$ in the SM EFT have to be included in the analysis. Otherwise, the analysis is inconsistent.

Our aim is not to perform a precision analysis, but to simply outline some issues that a precision Higgs and electroweak phenomenology program should take into account, and how the one-loop RGE result aids in this program. Some aspects of how the SM EFT modifies SM phenomenology have been discussed previously in refs. [20, 56, 63, 64] and other works. However, many aspects of how the SM EFT affects precision predictions have not been discussed in detail before, and we outline some of them below.

The Lagrangian of the SM EFT is

$$
\mathcal{L}=\mathcal{L}_{\mathrm{SM}}+\mathcal{L}^{(6)}+\ldots
$$

where the ... denote operators of dimension greater than six suppressed by additional powers of $\Lambda$. The dimension-six terms $\mathcal{L}^{(6)}$ can be treated perturbatively, i.e. we only need to include these to first order, since second-order contributions from $\mathcal{L}^{(6)}$ are as important as first-order contributions from $\mathcal{L}^{(8)}$, etc. The SM Lagrangian is

$$
\begin{aligned}
\mathcal{L}_{\mathrm{SM}}= & -\frac{1}{4} G_{\mu \nu}^{A} G^{A \mu \nu}-\frac{1}{4} W_{\mu \nu}^{I} W^{I \mu \nu}-\frac{1}{4} B_{\mu \nu} B^{\mu \nu}+\left(D_{\mu} H^{\dagger}\right)\left(D^{\mu} H\right)+\sum_{\psi=q, u, d, l, e} \bar{\psi} i \not D \psi \\
& -\lambda\left(H^{\dagger} H-\frac{1}{2} v^{2}\right)^{2}-\left[H^{\dagger j} \bar{d} Y_{d} q_{j}+\widetilde{H}^{\dagger j} \bar{u} Y_{u} q_{j}+H^{\dagger j} \bar{e} Y_{e} l_{j}+\text { h.c. }\right]
\end{aligned}
$$

and $\mathcal{L}^{(6)}$ is defined in eq. (2.1). We start by discussing the modification of the SM parameters at tree-level due to $\mathcal{L}^{(6)}$.

\subsection{Higgs mass and self-couplings}

The dimension-six Lagrangian of the SM EFT alters the definition of SM parameters at tree level in a number of ways. The operator $Q_{H}$ changes the shape of the scalar doublet potential at order $v^{2} / \Lambda^{2}$ to

$$
V(H)=\lambda\left(H^{\dagger} H-\frac{1}{2} v^{2}\right)^{2}-C_{H}\left(H^{\dagger} H\right)^{3},
$$

yielding the new minimum

$$
\left\langle H^{\dagger} H\right\rangle=\frac{v^{2}}{2}\left(1+\frac{3 C_{H} v^{2}}{4 \lambda}\right) \equiv \frac{1}{2} v_{T}^{2},
$$

on expanding the exact solution $\left(\lambda-\sqrt{\lambda^{2}-3 C_{H} \lambda v^{2}}\right) /\left(3 C_{H}\right)$ to first order in $C_{H}$. The shift in the vacuum expectation value (VEV) is proportional to $C_{H} v^{2}$, which is of order $v^{2} / \Lambda^{2}$.

The scalar field can be written in unitary gauge as

$$
H=\frac{1}{\sqrt{2}}\left(\begin{array}{c}
0 \\
{\left[1+c_{H, \mathrm{kin}}\right] h+v_{T}}
\end{array}\right),
$$


where

$$
c_{H, \mathrm{kin}} \equiv\left(C_{H \square}-\frac{1}{4} C_{H D}\right) v^{2}, \quad v_{T} \equiv\left(1+\frac{3 C_{H} v^{2}}{8 \lambda}\right) v .
$$

The coefficient of $h$ in eq. (5.5) is no longer unity, in order for the Higgs boson kinetic term to be properly normalized when the dimension-six operators are included. The kinetic terms

$$
\mathcal{L}=\left(D_{\mu} H^{\dagger}\right)\left(D^{\mu} H\right)+C_{H \square}\left(H^{\dagger} H\right) \square\left(H^{\dagger} H\right)+C_{H D}\left(H^{\dagger} D^{\mu} H\right)^{*}\left(H^{\dagger} D_{\mu} H\right),
$$

and the potential in eq. (5.3) yield ${ }^{6}$

$$
\begin{aligned}
& \mathcal{L}=\frac{1}{2}\left(\partial_{\mu} h\right)^{2}-\frac{c_{H, \text { kin }}}{v_{T}^{2}}\left[h^{2}\left(\partial_{\mu} h\right)^{2}+2 v h\left(\partial_{\mu} h\right)^{2}\right]-\lambda v_{T}^{2}\left(1-\frac{3 C_{H} v^{2}}{2 \lambda}+2 c_{H, \text { kin }}\right) h^{2} \\
& -\lambda v_{T}\left(1-\frac{5 C_{H} v^{2}}{2 \lambda}+3 c_{H, \text { kin }}\right) h^{3}-\frac{1}{4} \lambda\left(1-\frac{15 C_{H} v^{2}}{2 \lambda}+4 c_{H, \text { kin }}\right) h^{4}+\frac{3}{4} C_{H} v h^{5}+\frac{1}{8} C_{H} h^{6},
\end{aligned}
$$

for the $h$ self-interactions. The Higgs boson mass is

$$
m_{H}^{2}=2 \lambda v_{T}^{2}\left(1-\frac{3 C_{H} v^{2}}{2 \lambda}+2 c_{H, \text { kin }}\right) .
$$

\subsection{Yukawa couplings}

The definition of the fermion mass matrices and the Yukawa matrices are modified by the presence of $\psi^{2} H^{3}$ operators. The Lagrangian terms in the unbroken theory

$$
\begin{aligned}
\mathcal{L}= & -\left[H^{\dagger j} \bar{d}_{r}\left[Y_{d}\right]_{r s} q_{j s}+\widetilde{H}^{\dagger j} \bar{u}_{r}\left[Y_{u}\right]_{r s} q_{j s}+H^{\dagger j} \bar{e}_{r}\left[Y_{e}\right]_{r s} l_{j s}+\text { h.c. }\right] \\
& +\left[C_{d H}^{*}\left(H^{\dagger} H\right) H^{\dagger j} \bar{d}_{r} q_{j s}+C_{s r}^{*}\left(H^{\dagger} H\right) \tilde{H}^{\dagger j} \bar{u}_{r} q_{j s}+C_{s r}^{*}\left(H^{\dagger} H\right) H^{\dagger j} \bar{e}_{r} l_{j s}+\text { h.c. }\right]
\end{aligned}
$$

yield the fermion mass matrices

$$
\left[M_{\psi}\right]_{r s}=\frac{v_{T}}{\sqrt{2}}\left(\left[Y_{\psi}\right]_{r s}-\frac{1}{2} v^{2} C_{\psi H}^{*}\right), \quad \psi=u, d, e
$$

in the broken theory. The coupling matrices of the $h$ boson to the fermions $\mathcal{L}=-h \bar{u} \mathcal{Y} q+$ ... are

$$
\begin{aligned}
{\left[\mathcal{Y}_{\psi}\right]_{r s} } & =\frac{1}{\sqrt{2}}\left[Y_{\psi}\right]_{r s}\left[1+c_{H, \mathrm{kin}}\right]-\frac{3}{2} v^{2} C_{\psi H}^{*} \\
& =\frac{1}{v_{T}}\left[M_{\psi}\right]_{r s}\left[1+c_{H, \mathrm{kin}}\right]-v^{2} C_{\psi r}^{*}, \quad \psi=u, d, e
\end{aligned}
$$

and are not simply proportional to the fermion mass matrices, as is the case in the SM. In general, the fermion mass matrices and Yukawa matrices will not be simultaneously diagonalizable (these parameters have different RGEs), so that the couplings of the Higgs boson to the fermions will not be diagonal in flavor due to terms of order $v^{2} / \Lambda^{2}$.

\footnotetext{
${ }^{6}$ One can always replace $v$ by $v_{T}$ in terms that depend on the $\mathcal{L}^{(6)}$ coefficients, since the change is order $1 / \Lambda^{4}$.
} 


\section{$5.3 G_{F}$}

The value of the VEV in the SM is obtained from the measurement of $G_{F}$ in $\mu$ decay, $\mu^{-} \rightarrow e^{-}+\bar{\nu}_{e}+\nu_{\mu}$. Define the local effective interaction for muon decay as

$$
\mathcal{L}_{G_{F}}=-\frac{4 \mathcal{G}_{F}}{\sqrt{2}}\left(\bar{\nu}_{\mu} \gamma^{\mu} P_{L} \mu\right)\left(\bar{e} \gamma_{\mu} P_{L} \nu_{e}\right)
$$

The parameter $\mathcal{G}_{F}$ is fixed by measuring the muon lifetime. In the SM EFT, ${ }^{7}$

$$
-\frac{4 \mathcal{G}_{F}}{\sqrt{2}}=-\frac{2}{v_{T}^{2}}+\left(C_{\mu e e \mu}^{l l}+C_{e \mu \mu e}^{l l}\right)-2\left({\underset{e e}{C l}}_{e e}^{(3)}+C_{\mu \mu}^{(3)}\right) .
$$

The $C_{l l}$ terms are from the four-lepton interaction in $\mathcal{L}^{(6)}$, and the $C_{H l}^{(3)}$ terms are from $W$ exchange, where one $W \bar{l} \nu$ vertex is from the $Q_{H l}^{(3)}$ operator, and the other is the usual SM vertex. There are contributions to $\mu$ decay from $C_{\mu e r s}^{l l}$, and $C_{r s \mu e}^{l l}$ with $r \neq e, s \neq \mu$, as well as from $(\bar{L} L)(\bar{R} R)$ currents, but these do not interfere with the SM amplitude, and their contributions to the muon lifetime are higher order in $1 / \Lambda$.

Similar expressions hold for other weak decay processes, and $\mathcal{G}_{F}$ in $\tau$ decay, or in quark decays, can differ from $\mu$ decay due to the $C_{l l}$ and $C_{H l}^{(3)}$ terms.

\subsection{Gauge boson masses and couplings}

The definition of the gauge fields and the gauge couplings are affected by the dimension-six terms. The relevant dimension-six Lagrangian terms are

$$
\begin{aligned}
\mathcal{L}^{(6)}= & C_{H G} H^{\dagger} H G_{\mu \nu}^{A} G^{A \mu \nu}+C_{H W} H^{\dagger} H W_{\mu \nu}^{I} W^{I \mu \nu}+C_{H B} H^{\dagger} H B_{\mu \nu} B^{\mu \nu}+C_{H W B} H^{\dagger} \tau^{I} H W_{\mu \nu}^{I} B^{\mu \nu} \\
& +C_{G} f^{A B C} G_{\mu}^{A \nu} G_{\nu}^{B \rho} G_{\rho}^{C \mu}+C_{W} \epsilon^{I J K} W_{\mu}^{I \nu} W_{\nu}^{J \rho} W_{\rho}^{K \mu}
\end{aligned}
$$

In the broken theory, the $X^{2} H^{2}$ operators contribute to the gauge kinetic energies,

$$
\begin{aligned}
\mathcal{L}_{S M}+\mathcal{L}^{(6)}= & -\frac{1}{2} W_{\mu \nu}^{+} W_{-}^{\mu \nu}-\frac{1}{4} W_{\mu \nu}^{3} W_{3}^{\mu \nu}-\frac{1}{4} B_{\mu \nu} B^{\mu \nu}-\frac{1}{4} G_{\mu \nu}^{A} G^{A \mu \nu}+\frac{1}{2} v_{T}^{2} C_{H G} G_{\mu \nu}^{A} G^{A \mu \nu}, \\
& +\frac{1}{2} v_{T}^{2} C_{H W} W_{\mu \nu}^{I} W^{I \mu \nu}+\frac{1}{2} v_{T}^{2} C_{H B} B_{\mu \nu} B^{\mu \nu}-\frac{1}{2} v_{T}^{2} C_{H W B} W_{\mu \nu}^{3} B^{\mu \nu}
\end{aligned}
$$

so the gauge fields in the Lagrangian are not canonically normalized, and the last term in eq. (5.16) leads to kinetic mixing between $W^{3}$ and $B$. The mass terms for the gauge bosons from $\mathcal{L}_{\mathrm{SM}}$ and $\mathcal{L}^{(6)}$ are

$$
\mathcal{L}=\frac{1}{4} g_{2}^{2} v_{T}^{2} W_{\mu}^{+} W^{-\mu}+\frac{1}{8} v_{T}^{2}\left(g_{2} W_{\mu}^{3}-g_{1} B_{\mu}\right)^{2}+\frac{1}{16} v_{T}^{4} C_{H D}\left(g_{2} W_{\mu}^{3}-g_{1} B_{\mu}\right)^{2} .
$$

The gauge fields need to be redefined, so that the kinetic terms are properly normalized and diagonal. The first step is to redefine the gauge fields

$$
G_{\mu}^{A}=\mathcal{G}_{\mu}^{A}\left(1+C_{H G} v_{T}^{2}\right), \quad W_{\mu}^{I}=\mathcal{W}_{\mu}^{I}\left(1+C_{H W} v_{T}^{2}\right), \quad B_{\mu}=\mathcal{B}_{\mu}\left(1+C_{H B} v_{T}^{2}\right) .
$$

\footnotetext{
${ }^{7} e$ and $\mu$ are generation indices 1 and 2 , and are not summed over.
} 
The modified coupling constants are

$$
\bar{g}_{3}=g_{3}\left(1+C_{H G} v_{T}^{2}\right), \quad \bar{g}_{2}=g_{2}\left(1+C_{H W} v_{T}^{2}\right), \quad \bar{g}_{1}=g_{1}\left(1+C_{H B} v_{T}^{2}\right),
$$

so that the products $g_{3} G_{\mu}^{A}=\bar{g}_{3} \mathcal{G}_{\mu}^{A}$, etc. are unchanged. This takes care of the gluon terms. The electroweak terms are

$$
\begin{aligned}
\mathcal{L}= & -\frac{1}{2} \mathcal{W}_{\mu \nu}^{+} \mathcal{W}_{-}^{\mu \nu}-\frac{1}{4} \mathcal{W}_{\mu \nu}^{3} \mathcal{W}_{3}^{\mu \nu}-\frac{1}{4} \mathcal{B}_{\mu \nu} \mathcal{B}^{\mu \nu}-\frac{1}{2}\left(v_{T}^{2} C_{H W B}\right) \mathcal{W}_{\mu \nu}^{3} \mathcal{B}^{\mu \nu}+\frac{1}{4} \bar{g}_{2}^{2} v_{T}^{2} \mathcal{W}_{\mu}^{+} \mathcal{W}^{-\mu} \\
& +\frac{1}{8} v_{T}^{2}\left(\bar{g}_{2} \mathcal{W}_{\mu}^{3}-\bar{g}_{1} \mathcal{B}_{\mu}\right)^{2}+\frac{1}{16} v_{T}^{4} C_{H D}\left(\bar{g}_{2} \mathcal{W}_{\mu}^{3}-\bar{g}_{1} \mathcal{B}_{\mu}\right)^{2}
\end{aligned}
$$

The mass eigenstate basis is given by [65]

$$
\left[\begin{array}{c}
\mathcal{W}_{\mu}^{3} \\
\mathcal{B}_{\mu}
\end{array}\right]=\left[\begin{array}{cc}
1 & -\frac{1}{2} v_{T}^{2} C_{H W B} \\
-\frac{1}{2} v_{T}^{2} C_{H W B} & 1
\end{array}\right]\left[\begin{array}{cc}
\cos \bar{\theta} & \sin \bar{\theta} \\
-\sin \bar{\theta} & \cos \bar{\theta}
\end{array}\right]\left[\begin{array}{c}
\mathcal{Z}_{\mu} \\
\mathcal{A}_{\mu}
\end{array}\right],
$$

where the rotation angle is

$$
\tan \bar{\theta}=\frac{\bar{g}_{1}}{\bar{g}_{2}}+\frac{v_{T}^{2}}{2} C_{H W B}\left[1-\frac{\bar{g}_{1}^{2}}{\bar{g}_{2}^{2}}\right],
$$

so that

$$
\begin{aligned}
& \sin \bar{\theta}=\frac{\bar{g}_{1}}{\sqrt{\bar{g}_{1}^{2}+\bar{g}_{2}^{2}}}\left[1+\frac{v_{T}^{2}}{2} \frac{\bar{g}_{2}}{\bar{g}_{1}} \frac{\bar{g}_{2}^{2}-\bar{g}_{1}^{2}}{\bar{g}_{2}{ }^{2}+\bar{g}_{1}^{2}} C_{H W B}\right], \\
& \cos \bar{\theta}=\frac{\bar{g}_{2}}{\sqrt{\bar{g}_{1}{ }^{2}+\bar{g}_{2}{ }^{2}}}\left[1-\frac{v_{T}^{2}}{2} \frac{\bar{g}_{1}}{\bar{g}_{2}} \frac{\bar{g}_{2}{ }^{2}-\bar{g}_{1}{ }^{2}}{\bar{g}_{2}{ }^{2}+\bar{g}_{1}{ }^{2}} C_{H W B}\right] \text {. }
\end{aligned}
$$

The photon is massless, as it must be by gauge invariance, since $\mathrm{U}(1)_{Q}$ is unbroken. The $W$ and $Z$ masses are

$$
\begin{aligned}
M_{W}^{2} & =\frac{\bar{g}_{2}^{2} v_{T}^{2}}{4} \\
M_{Z}^{2} & =\frac{v_{T}^{2}}{4}\left(\bar{g}_{1}{ }^{2}+\bar{g}_{2}{ }^{2}\right)+\frac{1}{8} v_{T}^{4} C_{H D}\left(\bar{g}_{1}{ }^{2}+\bar{g}_{2}{ }^{2}\right)+\frac{1}{2} v_{T}^{4} \bar{g}_{1} \bar{g}_{2} C_{H W B} .
\end{aligned}
$$

The covariant derivative is

$$
D_{\mu}=\partial_{\mu}+i \frac{\bar{g}_{2}}{\sqrt{2}}\left[\mathcal{W}_{\mu}^{+} T^{+}+\mathcal{W}_{\mu}^{-} T^{-}\right]+i g_{Z}\left[T_{3}-\bar{s}^{2} Q\right] \mathcal{Z}_{\mu}+i \bar{e} Q \mathcal{A}_{\mu}
$$

where $Q=T_{3}+Y$, and the effective couplings are given by

$$
\begin{aligned}
& \bar{e}=\frac{\bar{g}_{1} \bar{g}_{2}}{\sqrt{\bar{g}_{2}^{2}+\bar{g}_{1}^{2}}}\left[1-\frac{\bar{g}_{1} \bar{g}_{2}}{\bar{g}_{2}^{2}+\bar{g}_{1}^{2}} v_{T}^{2} C_{H W B}\right]=\bar{g}_{2} \sin \bar{\theta}-\frac{1}{2} \cos \bar{\theta} \bar{g}_{2} v_{T}^{2} C_{H W B}, \\
& \bar{g}_{Z}=\sqrt{\bar{g}_{2}^{2}+\bar{g}_{1}^{2}}+\frac{\bar{g}_{1} \bar{g}_{2}}{\sqrt{\bar{g}_{2}^{2}+\bar{g}_{1}^{2}}} v_{T}^{2} C_{H W B}=\frac{\bar{e}}{\sin \bar{\theta} \cos \bar{\theta}}\left[1+\frac{\bar{g}_{1}{ }^{2}+\bar{g}_{2}{ }^{2}}{2 \bar{g}_{1} \bar{g}_{2}} v_{T}^{2} C_{H W B}\right] \text {, } \\
& \bar{s}^{2}=\sin ^{2} \bar{\theta}=\frac{\bar{g}_{1}{ }^{2}}{\bar{g}_{2}{ }^{2}+\bar{g}_{1}{ }^{2}}+\frac{\bar{g}_{1} \bar{g}_{2}\left(\bar{g}_{2}{ }^{2}-\bar{g}_{1}{ }^{2}\right)}{\left(\bar{g}_{1}{ }^{2}+\bar{g}_{2}{ }^{2}\right)^{2}} v_{T}^{2} C_{H W B} .
\end{aligned}
$$


The $\rho$ parameter, defined as the ratio of charged and neutral currents at low energies [66], is

$$
\rho \equiv \frac{\bar{g}_{2}^{2} M_{Z}^{2}}{\bar{g}_{Z}^{2} M_{W}^{2}}=1+\frac{1}{2} v_{T}^{2} C_{H D} .
$$

Measurements of the $W$ and $Z$ masses and couplings, and the photon coupling fix $\bar{g}_{1}, \bar{g}_{2}$, $v_{T}, C_{H W B}$ and $C_{H D}$. The couplings of the gauge bosons to fermions are also modified, and a recent discussion can be found in ref. [67].

\subsection{RGE for $C_{H}, C_{H D}, C_{H \square}$}

The discussion in sections 5.1-5.4 studied the impact of higher dimensional operators on the measured SM parameters at tree level. The coefficients $C_{H D}$, etc. of the higher dimension operators that enter the expressions are renormalized at the low scale, and are related to the parameters at the high scale $\Lambda$ by the RGE. As mentioned earlier, the RGE contributions are the same as the $\log \Lambda / m_{H}$ enhanced contributions from the finite parts of the one-loop diagrams.

The RGE for $C_{H}, C_{H D}$ and $C_{H \square}$ which enter the Higgs and gauge Lagrangian are

$$
\begin{aligned}
& \dot{C}_{H}=\left(108 \lambda+6 Y(S)-\frac{9}{2} g_{1}^{2}-\frac{27}{2} g_{2}^{2}\right) C_{H}-12 g_{1}^{2} \mathrm{y}_{H}^{2}\left(4 g_{1}^{2} \mathrm{y}_{H}^{2}+g_{2}^{2}-4 \lambda\right) C_{H B} \\
& -3 g_{2}^{2}\left(4 g_{1}^{2} \mathrm{y}_{H}^{2}+3 g_{2}^{2}-12 \lambda\right) C_{H W}-6 g_{1} g_{2} \mathrm{y}_{H}\left(4 g_{1}^{2} \mathrm{y}_{H}^{2}+g_{2}^{2}-4 \lambda\right) C_{H W B} \\
& -\frac{3}{4}\left(\left(4 \mathrm{y}_{H}^{2} g_{1}^{2}+g_{2}^{2}\right)^{2}+8\left(g_{2}^{2}-4 g_{1}^{2} \mathrm{y}_{H}^{2}\right) \lambda-64 \lambda^{2}\right) C_{H D} \\
& +\frac{40}{3}\left(g_{2}^{2} \lambda-12 \lambda^{2}\right) C_{H \square}+\frac{16 g_{2}^{2} \lambda}{3} C_{t t}^{(3)}+16 g_{2}^{2} \lambda C_{t t}^{(3)}+8 \lambda\left(\eta_{1}+\eta_{2}\right) \\
& -4\left(\left[Y_{e} Y_{e}^{\dagger} Y_{e}\right]_{w v} C_{e w}+3\left[Y_{d} Y_{d}^{\dagger} Y_{d}\right]_{w v} C_{v w}+3\left[Y_{u} Y_{u}^{\dagger} Y_{u}\right]_{w v} C_{u w}+\text { h.c. }\right) \\
& \dot{C}_{H \square}=\left(-\frac{16}{3} \mathrm{y}_{H}^{2} g_{1}^{2}-4 g_{2}^{2}+24 \lambda+4 Y(S)\right) C_{H \square}+2 g_{2}^{2} C_{t t}^{(3)}+2 g_{2}^{2} N_{c} C_{t t}^{(3)}+\frac{20}{3} g_{1}^{2} \mathrm{y}_{H}^{2} C_{H D} \\
& +\frac{4 g_{1}^{2} \mathrm{y}_{H}}{3}\left(N_{c} \mathrm{y}_{d} C_{t t}^{H d}+\mathrm{y}_{e} C_{t t}^{H e}+2 \mathrm{y}_{l} C_{t t}^{(1)}+2 N_{c} \mathrm{y}_{q} C_{t t}^{(1)}+N_{c} \mathrm{y}_{u} C_{t t}^{(1)}\right)-2 \eta_{3}, \\
& \dot{C}_{H D}=\left(-\frac{10}{3} \mathrm{y}_{H}^{2} g_{1}^{2}+\frac{9}{2} g_{2}^{2}+12 \lambda+4 Y(S)\right) C_{H D}+\frac{80}{3} g_{1}^{2} \mathrm{y}_{H}^{2} C_{H \square} \\
& +\frac{16 g_{1}^{2} \mathrm{y}_{H}}{3}\left(N_{c} \mathrm{y}_{d} C_{t t}+\mathrm{y}_{e} C_{t t}^{H e}+2 \mathrm{y}_{l} C_{t t}^{(1)}+2 N_{c} \mathrm{y}_{q} C_{t t}^{(1)}+N_{c} \mathrm{y}_{u} C_{t t}^{(1)}\right)-2 \eta_{4},
\end{aligned}
$$

where $\eta_{1,2,3,4}$ are defined in our previous paper ref. [10]. The precision electroweak parameter $T$ is $C_{H D}$, so these RGE are also used in section 5.11. Note that the dimension-six operator coefficients from the operators in parentheses on the second lines of eqs. (5.29) and (5.30) drop out of the running of the combination $\left(C_{H \square}-C_{H D} / 4\right)$ appearing in $c_{H, \text { kin }}$. The RGE for $C_{H W B}$ is given in section 5.11.

The RGE in eqs. (5.28)-(5.30) depend on other coefficients in $\mathcal{L}^{(6)}$. If the scale $\Lambda$ is a few $\mathrm{TeV}$, the RGE can be integrated perturbatively, so that

$$
C(\mu) \approx C(\Lambda)-\frac{1}{16 \pi^{2}} \gamma_{C} \ln \frac{\Lambda}{\mu}+\ldots \quad \text { where } \quad \dot{C}=\gamma_{C},
$$


and the $\ldots$ are part of the leading-log series $\gamma_{C}^{2} \ln ^{2} \Lambda / \mu$ given by exact integration of the RGE. The $\ln \Lambda / \mu$ terms in eq. (5.31) must be the same as the $\ln \mu$ terms in the finite parts of the one-loop graphs. Thus the anomalous dimensions are another way of computing the $\ln \Lambda / \mu$ enhanced terms in the finite parts of the one-loop graphs.

\section{$5.6 \quad h \rightarrow f \bar{f}$}

The decay of the Higgs boson into fermions is another important test of the symmetry breaking structure of the SM. Define the effective coupling $\mathcal{Y}_{b}$ of the $b$ quark to the Higgs by $\mathcal{L}_{\text {Yuk }}=-\mathcal{Y}_{b} h \bar{b} b$. The decay width is given by

$$
\Gamma(h \rightarrow \bar{b} b)=\frac{\mathcal{Y}_{b}^{2} m_{H} N_{c}}{8 \pi}\left(1-4 \frac{m_{b}^{2}}{m_{H}^{2}}\right)^{3 / 2},
$$

where all parameters are renormalized at $\mu \sim m_{H}$.

In the SM, the effective coupling of the $b$ quark to the Higgs field can be predicted very accurately. The $b$-quark mass can be determined very precisely from global studies of $\bar{B} \rightarrow X_{c} \ell \bar{\nu}$ and $X_{s} \gamma$ [68], and then used to determine the $b$-quark Yukawa coupling at the scale $m_{H}$ using the SM RGE. The relation $\mathcal{Y}_{b}=\sqrt{2} m_{b} / v$ between the Higgs coupling and quark mass is modified in the SM EFT, and is given by eq. (5.12), with $\mathcal{Y}_{b}=\left[\mathcal{Y}_{d}\right]_{b b}$, and the relation between $v$ and $G_{F}$ is modified as in eq. (5.14) due to tree level effects from $\mathcal{L}^{(6)}$.

The scaling of parameters from $m_{b}$ to $m_{H}$ is also modified. The dimension-six operator contribution to the one-loop running of the effective coupling of the SM Higgs to fermions is given in ref. [12]. We repeat the result for the down quarks here for the sake of completeness. ${ }^{8}$ The running of the $Y_{d}$ is modified by the terms

$$
\begin{aligned}
& \mu \frac{\mathrm{d}}{\mathrm{d} \mu}\left[Y_{d}\right]_{r s}=\frac{m_{H}^{2}}{16 \pi^{2}}\left[3 C_{d H}^{*}-C_{H \square}\left[Y_{d}\right]_{r s}+\frac{1}{2} C_{H D}\left[Y_{d}\right]_{r s}+\left[Y_{d}\right]_{r t}\left(C_{t s}^{(1)}+3 C_{t s}^{(3)}\right)-C_{r t}^{C_{t}}\left[Y_{d}\right]_{t s}\right. \\
& -\left[Y_{u}\right]_{t s} C_{t r u d}^{*}-2\left(C_{t r}^{(1) *}+c_{F, 3} C_{\substack{q d \\
\text { sptr }}}^{(8) *}\right)\left[Y_{d p t r}\right]_{t p}+\underset{p t r s}{C_{l e d q}}\left[Y_{e}\right]_{p t}^{*}+N_{c} C_{q u q d}^{(1) *}\left[Y_{u}\right]_{t p}^{*} \\
& \left.+\frac{1}{2}\left(C_{\substack{q u q d \\
\text { sptr }}}^{(1) *}+c_{F, 3} C_{\text {quqd }}^{(8) *}\right)\left[Y_{u}\right]_{t p}^{*}\right]
\end{aligned}
$$

These terms are of order $v^{2} / \Lambda^{2}$, and are just as important as the running of the $C_{\psi H}$ and $c_{H, \text { kin }}$ contributions in eq. (5.12), and must be included for a consistent calculation.

The net effect of including the RGE in eq. (5.12) and eq. (5.33) is to introduce a shift of the form

$$
\Gamma(h \rightarrow \bar{b} b)=\frac{\left(\mathcal{Y}_{b}+\Delta \mathcal{Y}_{b}\right)^{2} m_{H} N_{c}}{8 \pi}\left(1-4 \frac{m_{b}^{2}}{m_{H}^{2}}\right)^{3 / 2},
$$

where the running effects induced by new physics are included in $\Delta \mathcal{Y}_{b}$ :

$$
\Delta \mathcal{Y}_{b}=\frac{m_{H}^{2}}{16 \pi^{2}} \log \left(\frac{m_{H}}{m_{b}}\right) C_{1}+\frac{m_{H}^{2}}{16 \pi^{2}} \log \left(\frac{m_{H}}{\Lambda}\right) C_{2} .
$$

\footnotetext{
${ }^{8}$ Note that the usual one loop running of the SM parameters summarized in ref. [69-71] should be added to this result for the full scale dependence of these effective couplings in the SM EFT.
} 
The expression for $C_{1}$ is obtained by setting $r=s=3$ in the expression in square brackets on the r.h.s. of eq. (5.33). The expression for $C_{2}$ is

$$
C_{2}=\frac{1}{2 \sqrt{2} \lambda}\left[Y_{d}\right]_{33}\left(\dot{C}_{H \square}-\frac{1}{4} \dot{C}_{H D}\right)-\frac{3}{4 \lambda} \dot{C}_{d H}^{*}
$$

where the anomalous dimensions $\dot{C}_{H \square}, \dot{C}_{H D}$, and $\dot{C}_{d H}$ are given in eqs. (5.29), (5.30) and section C.5 respectively. Note that as we are considering $\Lambda \sim \mathrm{TeV}$, the log enhancement is modest and of about the same size for running from $m_{b}$ to $m_{H}$ and from $\Lambda$ to $m_{H}$. The $\log \left(m_{H} / m_{b}\right)$ contribution in eq. (5.35), and analogous terms in other amplitudes, have been neglected in ref. [20], and need to be included for a consistent calculation including $1 / \Lambda^{2}$ RGE effects.

The discussion above also applies to Higgs decays into other fermions, such as $c \bar{c}$ and $\tau^{+} \tau^{-}$. Using newly developed charm tagging techniques [72], it may be possible to measure deviations in $\Gamma(h \rightarrow \bar{c} c)$ at the LHC (see the discussion in ref. [73]).

There are also flavor-changing Higgs-fermion couplings from $\mathcal{L}^{(6)}$, which contribute to flavor-changing Higgs decays, such as $h \rightarrow b \bar{s}$. These do not interfere with the SM Higgs amplitude, which is flavor diagonal, so the flavor-changing decay rates are order $1 / \Lambda^{4}$. Nevertheless, as the running of $C_{e H}, C_{d H}$ and $C_{u H}$ is not the same as the running of the SM Yukawa couplings, searches for Higgs flavor violation is well-motivated. For some recent work on this subject, see refs. [74, 75].

\section{$5.7 \quad h \rightarrow W W$ and $h \rightarrow Z Z$}

The $h \rightarrow W W$ and $h \rightarrow Z Z$ amplitudes receive direct contributions from $\mathcal{L}^{(6)}$. The relevant $C P$-even Lagrangian terms are

$$
\begin{aligned}
\mathcal{L}= & \left(D_{\mu} H\right)^{\dagger}\left(D^{\mu} H\right)-\frac{1}{4}\left(W_{\mu \nu}^{I} W^{I \mu \nu}+B_{\mu \nu} B^{\mu \nu}\right), \\
& +C_{H W} Q_{H W}+C_{H B} Q_{H B}+C_{H W B} Q_{H W B}+C_{H D} Q_{H D},
\end{aligned}
$$

which lead to the interactions

$$
\mathcal{L}=\frac{1}{4} \bar{g}_{2}^{2} v_{T} h\left[\left(\mathcal{W}_{\mu}^{1}\right)^{2}+\left(\mathcal{W}_{\mu}^{2}\right)^{2}\right]\left[1+c_{H, \mathrm{kin}}\right]+C_{H W} v_{T} h\left[\left(\mathcal{W}_{\mu \nu}^{1}\right)^{2}+\left(\mathcal{W}_{\mu \nu}^{2}\right)^{2}\right]
$$

for the $W$, and

$$
\begin{aligned}
\mathcal{L}= & \frac{1}{4}\left(\bar{g}_{2}{ }^{2}+\bar{g}_{1}{ }^{2}\right) v_{T} h\left(\mathcal{Z}_{\mu}\right)^{2}\left[1+c_{H, \mathrm{kin}}+v_{T}^{2} C_{H D}\right]+\frac{1}{2} \bar{g}_{1} \bar{g}_{2} v_{T}^{3} h\left(\mathcal{Z}_{\mu}\right)^{2} C_{H W B} \\
& +v_{T} h\left(\mathcal{Z}_{\mu \nu}\right)^{2}\left(\frac{\bar{g}_{2}{ }^{2} C_{H W}+\bar{g}_{1}{ }^{2} C_{H B}+\bar{g}_{1} \bar{g}_{2} C_{H W B}}{\bar{g}_{2}{ }^{2}+\bar{g}_{1}{ }^{2}}+\frac{1}{2} \bar{g}_{1} \bar{g}_{2} C_{H W B}\right)
\end{aligned}
$$

for the $Z$.

A ratio of deviations in the SM gauge boson coupling to the Higgs, reported in [76], is defined as

$$
\lambda_{W Z} \equiv \frac{\Gamma(h \rightarrow W W)}{\Gamma(h \rightarrow W W)_{S M}} \frac{\Gamma(h \rightarrow Z Z)_{S M}}{\Gamma(h \rightarrow Z Z)}
$$


From eqs. (5.38), (5.39), we see that $c_{H, \text { kin }}$ cancels out in $\lambda_{W Z}$, but there are corrections from the Higgs-gauge operators $C_{H W}, C_{H B}$ and $C_{H W B}$. This correction depends on the off-shellness of the $W$ and $Z$, since it is proportional to the field-strength tensors, and thus momentum-dependent. In the SM EFT, the ratio $\lambda_{W Z}$ depends on the $\mathcal{L}^{(6)}$ parameters $C_{H W}, C_{H B}$ and which are not custodial $\mathrm{SU}(2)$ violating, as well as $C_{H W B}$ and $C_{H D}$ which are custodial $\mathrm{SU}(2)$ violating. The couplings of the gauge bosons to fermions are also modified. For a recent discussion on these corrections in this basis see ref. [67].

\section{$5.8 \quad g g \rightarrow h$}

The Higgs-gluon operators $Q_{H G}$ and $Q_{H \widetilde{G}}$ contribute to the Higgs production rate via gluon fusion. The $\mathcal{L}^{(6)}$ contribution to $g g \rightarrow h$ is important because the SM amplitude starts at one loop order, with no tree-level contribution. A similar enhancement of $\mathcal{L}^{(6)}$ corrections occurs for $h \rightarrow \gamma \gamma$ and $h \rightarrow \gamma Z$ discussed in the next two sections.

Define $\mathscr{C}_{g g}$ and $\widetilde{\mathscr{C}}_{g g}$ by rescaling $C_{H G}$ and $C_{H \widetilde{G}}$ by $g_{3}$,

$$
C_{H G}=g_{3}^{2} \mathscr{C}_{g g} \quad C_{H \widetilde{G}}=g_{3}^{2} \tilde{\mathscr{C}}_{g g} .
$$

The scaling by $g_{3}$ simplifies the RGE, and makes contact with the notation of refs. $[13,77]$ which uses

$$
\mathscr{C}_{g g}=-\frac{c_{G}}{2 \Lambda^{2}} \quad \tilde{\mathscr{C}}_{g g}=-\frac{\tilde{c}_{G}}{2 \Lambda^{2}}
$$

since a factor of $-1 /\left(2 \Lambda^{2}\right)$ was included in the normalization of the operators. The other advantage of the rescaling is that the field and coupling constant renormalizations eq. (5.18) and (5.19) cancel out.

The change in $g g \rightarrow h$ relative to the SM is given by [77]

$$
\frac{\sigma(g g \rightarrow h)}{\sigma^{\mathrm{SM}}(g g \rightarrow h)} \simeq \frac{\Gamma(h \rightarrow g g)}{\Gamma^{\mathrm{SM}}(h \rightarrow g g)} \simeq\left|1+\frac{16 \pi^{2} v^{2} \mathscr{C}_{g g}}{I^{g}}\right|^{2}+\left|\frac{16 \pi^{2} v^{2} \tilde{\mathscr{C}}_{g g}}{I^{g}}\right|^{2}
$$

where $I^{g} \approx 0.37$ is the numerical value of a Feynman parameter integral [77, 78]. We have neglected corrections from $c_{H, \text { kin }}$ and the Yukawa couplings eq. (5.12) which are $v^{2} / \Lambda^{2}$, but not enhanced by $16 \pi^{2}$. If $\mathscr{C}_{g g}$ from BSM physics is loop suppressed as in the SM, then these terms must be included.

The complete one loop RGE of $\mathscr{C}_{g g}$ and $\tilde{\mathscr{C}}_{g g}$ are relatively simple,

$$
\begin{aligned}
& \dot{\mathscr{C}}_{g g}=\left(12 \lambda+2 Y(S)-\frac{3}{2} g_{1}^{2}-\frac{9}{2} g_{2}^{2}\right) \mathscr{C}_{g g}-2\left(\left[Y_{d}\right]_{w v} \mathscr{C}_{d G}+\left[Y_{u}\right]_{w v} \mathscr{C}_{u G}+\text { h.c. }\right) \\
& \tilde{\mathscr{C}}_{g g}=\left(12 \lambda+2 Y(S)-\frac{3}{2} g_{1}^{2}-\frac{9}{2} g_{2}^{2}\right) \widetilde{\mathscr{C}}_{g g}+2\left(i\left[Y_{d}\right]_{w v} \mathscr{C}_{d G}+i\left[Y_{u w}\right]_{w v} \mathscr{C}_{v G}+\text { h.c. }\right)
\end{aligned}
$$

where

$$
C_{d G}=g_{3} \mathscr{C}_{d G}, \quad \quad C_{v w}, \quad=g_{v w} \mathscr{C}_{v G},
$$


are rescaled coefficients of the color magnetic dipole operators, and

$$
Y(S)=\operatorname{Tr}\left[N_{c} Y_{u}^{\dagger} Y_{u}+N_{c} Y_{d}^{\dagger} Y_{d}+Y_{e}^{\dagger} Y_{e}\right]
$$

The Higgs-gluon contributions in the first term of eq. (5.44) were computed in ref. [13]. The only new contribution from the full RGE is the second term from the color dipole operators which was also calculated in ref. [19] for the special case of no flavor indices, and with only a non-zero top quark Yukawa coupling.

\section{$5.9 h \rightarrow \gamma \gamma$}

A very important process is $h \rightarrow \gamma \gamma$, which played a key role in the discovery of the SM scalar. Again, it is convenient to define

$$
\mathscr{C}_{\gamma \gamma}=\frac{1}{g_{2}^{2}} C_{H W}+\frac{1}{g_{1}^{2}} C_{H B}-\frac{1}{g_{1} g_{2}} C_{H W B}
$$

in terms of which our previously defined coefficients [13, 77] are

$$
\mathscr{C}_{\gamma \gamma}=-\frac{c_{\gamma \gamma}}{2 \Lambda^{2}}, \quad \tilde{\mathscr{C}}_{\gamma \gamma}=-\frac{\tilde{c}_{\gamma \gamma}}{2 \Lambda^{2}}
$$

The $h \rightarrow \gamma \gamma$ rate is

$$
\frac{\Gamma(h \rightarrow \gamma \gamma)}{\Gamma^{\mathrm{SM}}(h \rightarrow \gamma \gamma)} \simeq\left|1+\frac{8 \pi^{2} v^{2} \mathscr{C}_{\gamma \gamma}}{I^{\gamma}}\right|^{2}+\left|\frac{8 \pi^{2} v^{2} \tilde{\mathscr{C}}_{\gamma \gamma}}{I^{\gamma}}\right|^{2}
$$

where $I^{\gamma} \approx-1.65$ is a Feynman parameter integral [77, 78]. Again, as in the gluon case, we are dropping $v^{2} / \Lambda^{2}$ that must be included if $\mathscr{C}_{\gamma \gamma}$ from BSM physics is loop suppressed.

The effective amplitude is

$$
\mathscr{C}_{\gamma \gamma} e^{2} F_{\mu \nu} F^{\mu \nu} h v
$$

where

$$
g_{1}=\frac{e}{\cos \theta_{W}} \quad g_{2}=\frac{e}{\sin \theta_{W}}
$$

are the definitions of $e$ and $\theta_{W}$ without a bar. These differ from the coupling constants in eq. (5.19) (with a bar) at order $1 / \Lambda^{2}$.

The complete one-loop RGE is

$$
\begin{aligned}
& \dot{\mathscr{C}}_{\gamma \gamma}=\left(12 \lambda-\frac{3}{2} g_{1}^{2}-\frac{9}{2} g_{2}^{2}+2 Y(S)\right) \mathscr{C}_{\gamma \gamma}+\left(8 \lambda-6 g_{2}^{2}\right) \frac{C_{H W B}}{g_{1} g_{2}} \\
& \left.-18 g_{2} C_{W}+\underset{r s}{4 \mathscr{C}_{d \gamma}}\left[Y_{d}\right]_{s r}+\underset{r s}{4 \mathscr{C O}_{\gamma}}\left[Y_{e}\right]_{s r}-\underset{r s}{8 \mathscr{C}_{u r}}\left[Y_{u}\right]_{s r}+\text { h.c. }\right), \\
& \dot{\widetilde{\mathscr{C}}}_{\gamma \gamma}=\left(12 \lambda-\frac{3}{2} g_{1}^{2}-\frac{9}{2} g_{2}^{2}+2 Y(S)\right) \widetilde{\mathscr{C}}_{\gamma \gamma}+\left(8 \lambda-6 g_{2}^{2}\right) \frac{C_{H \widetilde{W} B}}{g_{1} g_{2}} \\
& -18 g_{2} C_{\widetilde{W}}+\left(-4 i \mathscr{C}_{r \gamma}\left[Y_{d s}\right]_{s r}-4 i \mathscr{C}_{r s}\left[Y_{e}\right]_{s r}+\underset{r s}{8 i \mathscr{C}_{r \gamma}}\left[Y_{u}\right]_{s r}+\text { h.c. }\right) \text {. }
\end{aligned}
$$


The first line of each equation is the contribution from the $8 \times 8$ submatrix of Higgs-gauge operators computed in ref. [13]. The second line gives the additional terms including all 59 operators. There are contributions from the triple-gauge operators

$$
Q_{W}=\epsilon^{I J K} W_{\mu}^{I \nu} W_{\nu}^{J \rho} W_{\rho}^{K \mu}, \quad \quad Q_{\widetilde{W}}=\epsilon^{I J K} W_{\mu}^{I \nu} W_{\nu}^{J \rho}, \widetilde{W}_{\rho}^{K \mu}
$$

and the dipole operator coefficients defined in section 5.13.

This result is the first truly complete one-loop result of the RGE running of $\mathscr{C}_{\gamma \gamma}$.

\section{$5.10 \quad h \rightarrow \gamma Z$}

The measurement of $h \rightarrow \gamma Z$ at LHC has not yet reached the sensitivity required to observe the SM rate [79, 80]. Nevertheless, this process is interesting in several BSM scenarios because a suppression of BSM effects in $h \rightarrow \gamma \gamma, g g$ due to a pseudo-Goldstone Higgs does not necessarily imply a suppression of BSM effects in $h \rightarrow \gamma Z$ (for a recent discussion see [81]). We define the effective Wilson coefficient in this case to be

$$
\mathscr{C}_{\gamma Z}=\frac{1}{g_{1} g_{2}} C_{H W}-\frac{1}{g_{1} g_{2}} C_{H B}-\left(\frac{1}{2 g_{1}^{2}}-\frac{1}{2 g_{2}^{2}}\right) C_{H W B}
$$

so that the modification of the decay rate is

$$
\frac{\Gamma(h \rightarrow \gamma Z)}{\Gamma^{\mathrm{SM}}(h \rightarrow \gamma Z)} \simeq\left|1+\frac{8 \pi^{2} v^{2} \mathscr{C}_{\gamma Z}}{I^{Z}}\right|^{2}+\left|\frac{8 \pi^{2} v^{2} \tilde{\mathscr{C}}_{\gamma Z}}{I^{Z}}\right|^{2}
$$

$I^{Z} \approx-2.87[77,78]$, again neglecting $v^{2} / \Lambda^{2}$ terms due to $c_{H, \text { kin }}$, etc. and our previously defined coefficients are

$$
\mathscr{C}_{\gamma Z}=-\frac{c_{\gamma Z}}{2 \Lambda^{2}}, \quad \widetilde{\mathscr{C}}_{\gamma Z}=-\frac{\tilde{c}_{\gamma Z}}{2 \Lambda^{2}}
$$

The one loop RGE results for the CP-even term

$$
\begin{aligned}
& \dot{\mathscr{C}}_{\gamma Z}=\frac{1}{2} \csc \theta_{W} \sec \theta_{W}\left\{\left(2 \cos 2 \theta_{W}+1\right)\left[Y_{d}\right]_{w v} \mathscr{C}_{d \gamma} \underset{v w}{ }+\left(2 \cos 2 \theta_{W}-1\right)\left[Y_{e}\right]_{w v} \underset{\mathscr{C}_{e \gamma}}{v w}\right. \\
& \left.-\left(4 \cos 2 \theta_{W}-1\right)\left[Y_{u}\right]_{w v} \mathscr{C}_{v w}+\text { h.c. }\right\}+2\left(\left[Y_{d}\right]_{w v} \mathscr{C}_{d Z}+\left[Y_{e}\right]_{w v} \mathscr{C}_{v Z}-2\left[Y_{u}\right]_{w v} \mathscr{C}_{u Z}+\text { h.c. }\right) \\
& +\left(12 \lambda+2 Y(S)-\frac{22}{3} e^{2}+\frac{19}{3} e^{2} \sec ^{2} \theta_{W}-\frac{20}{3} e^{2} \csc ^{2} \theta_{W}\right) \mathscr{C}_{\gamma Z} \\
& +e^{2}\left(\frac{11}{3} \cos 2 \theta_{W}-10\right) \csc \theta_{W} \sec \theta_{W} \mathscr{C}_{\gamma \gamma}+e\left(\frac{3}{2} \sec \theta_{W}-\frac{33}{2} \cot \theta_{W} \csc \theta_{W}\right) C_{W} \\
& +\left(6 e^{2}-4 e^{2} \csc ^{2} \theta_{W}+4 \lambda \cos 2 \theta_{W}\right) \csc \theta_{W} \sec \theta_{W} \frac{C_{H W B}}{g_{1} g_{2}} .
\end{aligned}
$$

The RGE for $\dot{\widetilde{\mathscr{C}}}_{\gamma Z}$ is given by the substitution $Y_{\psi} \rightarrow-i Y_{\psi}, \mathscr{C}_{\gamma Z} \rightarrow \tilde{\mathscr{C}}_{\gamma Z}, \mathscr{C}_{\gamma \gamma} \rightarrow \tilde{\mathscr{C}}_{\gamma \gamma}$, $C_{W} \rightarrow C_{\widetilde{W}}$, and $C_{H W B} \rightarrow C_{H \widetilde{W} B}$, as for the $g g$ and $\gamma \gamma$ amplitudes. 


\subsection{Electroweak precision observables}

We are assuming $\Lambda$ is parametrically higher than the EW scale $v$, so the usual $S, T$ and $U$ parametrization [82-85] of the oblique electroweak precision data (EWPD) can be used. An operator based analysis of EWPD was first developed in ref. [65]. The standard operator based approach identifies the $S$ parameter with the operator $Q_{H W B}$, and the $T$ parameter with the operator $Q_{H D}$,

$$
S=\frac{16 \pi v^{2}}{g_{1} g_{2}} C_{H W B}, \quad T=-2 \pi v^{2}\left(\frac{1}{g_{1}^{2}}+\frac{1}{g_{2}^{2}}\right) C_{H D} .
$$

Note that the experimental constraints on $S, T$ are inferred from measurements with fermionic external states. These processes can have contributions from other higher dimensional operators. We assume, as is commonly done, that these other contributions are small in writing eq. (5.58). A shift in the definition of $v$ is order $1 / \Lambda^{4}$ for this expression, and we neglect this effect. The $U$ parameter corresponds to the dimension-eight operator $\left(H^{\dagger} W^{\mu \nu} H\right)\left(H^{\dagger} W_{\mu \nu} H\right)$, which we neglect. A fit that treats $m_{h}=126 \mathrm{GeV}$ as an input value [86] to EWPD finds $S=0.03 \pm 0.10$ and $T=0.05 \pm 0.12$ with a correlation coefficient between $S$ and $T$ of 0.89 .

$S$ and $T$ depend on $C_{H W B}$ and $C_{H D}$ evaluated at the weak scale. The RG evolution of $C_{H D}$ is given in eq. (5.30), and the RG evolution of $C_{H W B}$ is

$$
\begin{aligned}
& \dot{C}_{H W B}=\left(4 \lambda+2 Y(S)+\frac{4}{3} g_{2}^{2}+\frac{19}{3} g_{1}^{2}\right) C_{H W B}+2 g_{1} g_{2}\left(C_{H W}+C_{H B}\right)+3 g_{1} g_{2}^{2} C_{W} \\
& +g_{2}\left(3\left[Y_{u}\right]_{w v} C_{v w}-3\left[Y_{d w}\right]_{w v} C_{d B}-\left[Y_{e}\right]_{w v} C_{e B}+\text { h.c. }\right) \\
& +g_{1}\left(5\left[Y_{u}\right]_{w v} C_{v w}+\left[Y_{d}\right]_{w v} C_{d W}+3\left[Y_{e}\right]_{w v} C_{e w}+\text { h.c. }\right) \text {. }
\end{aligned}
$$

The $T$ parameter is usually interpreted as a measure of custodial symmetry violation, whereas the $S$ parameter is considered to be sensitive to the difference between the number of left-handed and right-handed fermions. Interestingly, the SM EFT one loop RGE does not mix the operators $C_{H W B}, C_{H D}$. However, this does not follow from custodial symmetry. The SM violates custodial symmetry in $g_{1}$ interactions, and through mass splittings of the $\mathrm{SU}(2)_{L}$ doublets. If we take the limit $Y_{d} \rightarrow Y_{u}, Y_{e} \rightarrow 0$ and $\mathrm{y}_{d} \rightarrow \mathrm{y}_{u}$, then $\mathrm{y}_{H} \rightarrow 0$ from eq. (2.9). In this limit, the standard model preserves custodial $\mathrm{SU}(2)$, as does the RGE. This provides a non-trivial check of our results.

The consequences of the RGE for precision electroweak parameters was studied in ref. [13]. The RGE allows one to compute the $\ln \Lambda / m_{H}$ contribution to these observables, which was computed previously in the broken theory [28-30]. Our computation agrees with their results for the terms they computed, but has additional effects (e.g. due to the top quark Yukawa) which were not in the previous results.

\subsection{Triple gauge boson couplings}

Another promising source of information on EW interactions are triple gauge couplings (TGC). For some recent studies on the phenomenology of these measurements see refs. [57, 
$63,87,88]$. Some of the scale dependence of the operators involved in this process (in another basis) has been determined [89, 90]. In the basis used here, a operator that contributes directly at tree level to TGC measurements is $Q_{W}$. (Other contributions come about indirectly due to field redefinitions, or a redefinition of input parameters.) The full RGE of the Wilson coefficient of the operator $Q_{W}$ has the simple form

$$
\dot{C}_{W}=\left(24-3 b_{0,2}\right) g_{2}^{2} C_{W}, \quad \text { or } \quad \mu \frac{\mathrm{d}}{\mathrm{d} \mu}\left(\frac{C_{W}}{g_{2}^{3}}\right)=24 g_{2}^{2}\left(\frac{C_{W}}{g_{2}^{3}}\right),
$$

where $b_{0,2}$ is the first coefficient in the $g_{2} \beta$-function. The triple gauge boson operators do not mix with any other dimension-six operators. This multiplicative renormalization can be largely understood using the results of ref. [11]. Consequently, TGC measurements provide a relatively clean and important probe of this dimension-six operator which contributes at tree level.

Recently, refs. [91, 92] have shown that the decay spectra of the three-body decay $h \rightarrow V \ell^{+} \ell^{-}$are particularly rich sources of information on the possible effects of anomalous couplings of the Higgs boson, and BSM contact interactions. The full decomposition of the modification of the $V \ell^{+} \ell^{-}$decay spectra in the operator basis used here was given in ref. [67], which shows that the relevant terms depend on the coefficients $C_{W B}, C_{H D}$, $C_{H W}, C_{H B}, C_{H l}^{1}, C_{H l}^{3}, C_{H e}$, as well as the coefficient $c_{H \text {,kin }}$ which only modifies the total decay rate.

It has been argued that TGC measurements probe the same physics as $h \rightarrow V \ell^{+} \ell^{-}$decays [57] in the SILH basis. This claim comes about by arbitrarily setting the operator $C_{W}$, which is present in the SILH basis, and in the analysis in ref. [57], to zero. This operator contributes to TGC measurements, but not to $h \rightarrow V \ell^{+} \ell^{-}$decays at tree level. It is by using this arbitrary choice that ref. [57] claims a strong relationship between these experimentally measurable quantities. This makes the results in ref. [57] model-dependent, and not general. For example, the exactly solvable model of ref. [93] produces $C_{W}$ but no Higgs-lepton operators. In the non-redundant basis of ref. [9], TGC measurements are also not related to $h \rightarrow V \ell^{+} \ell^{-}$decays since the combination of Wilson coefficients that contribute to the two processes is not identical. Measurable results are basis independent, and model independent results do not arbitrarily set operators to zero, as was done in ref. [57]. We disagree with the strong conclusions of ref. [57] which are stated as broad, model-independent, conclusions.

\section{$5.13 \mu \rightarrow e \gamma$, magnetic moments, and electric dipole moments}

The lepton dipole operators

$$
\mathcal{L}=\underset{r s}{C_{e W}} \bar{l}_{r, a} \sigma^{\mu \nu} e_{s} \tau_{a b}^{I} H_{b} W_{\mu \nu}^{I}+C_{r s} \bar{l}_{r, a} H_{a} \sigma^{\mu \nu} e_{s} H_{a} B_{\mu \nu}+\text { h.c. }
$$

contribute to radiative transitions such as $\mu \rightarrow e \gamma$ which is a remarkably clean window to physics BSM. In the broken phase, eq. (5.61) gives the charged lepton operators

$$
\mathcal{L}=\frac{e v}{\sqrt{2}} \underset{r s}{\mathscr{C}_{e \gamma}} \bar{e}_{r} \sigma^{\mu \nu} P_{R} e_{s} F_{\mu \nu}+\frac{e v}{\sqrt{2}} \mathscr{C}_{r s} \bar{e}_{r} \sigma^{\mu \nu} P_{R} e_{s} Z_{\mu \nu}+\text { h.c. }
$$


where $r$ and $s$ are flavor indices $\left(\left\{e_{e}, e_{\mu}, e_{\tau}\right\} \equiv\{e, \mu, \tau\}\right)$ and

$$
\begin{array}{rlrl}
\mathscr{C}_{e \gamma} & =\frac{1}{g_{1}} C_{e B}-\frac{1}{g_{2}} C_{e W} & \mathscr{C}_{e Z} & =-\frac{1}{g_{2}} C_{e B}-\frac{1}{g_{1}} C_{e W} \\
\mathscr{C}_{d \gamma} & =\frac{1}{g_{1}} C_{d B}-\frac{1}{g_{2}} C_{d W} & \mathscr{C}_{d Z} & =-\frac{1}{g_{2}} C_{d B}-\frac{1}{g_{1}} C_{d W} \\
r s & \mathscr{r}_{r s} \\
\mathscr{C}_{r s}=\frac{1}{g_{1}} C_{u B}+\frac{1}{g_{2}} C_{u W} & \mathscr{C}_{r z} & =-\frac{1}{g_{2}} C_{r s}+\frac{1}{g_{1}} C_{r S}
\end{array}
$$

$C_{u W}$ has the opposite sign for $u$-type quarks in eq. (5.63) because of the opposite sign for $T_{3 L}$. The RGE for $\mathscr{C}_{e \gamma}$ is

$$
\begin{aligned}
& \underset{r s}{\dot{\mathscr{C}} \gamma}=\left\{Y(s)+e^{2}\left(12-\frac{9}{4} \csc ^{2} \theta_{W}+\frac{1}{4} \sec ^{2} \theta_{W}\right)\right\} \underset{\mathscr{C}_{r s}}{\boldsymbol{C}_{s}} \\
& +\underset{r v}{2 \mathscr{C}_{e \gamma}}\left[Y_{e} Y_{e}^{\dagger}\right]_{v s}+\left(\frac{1}{2}-2 \cos ^{2} \theta_{W}\right)\left[Y_{e}^{\dagger} Y_{e}\right]_{r w} \mathscr{C}_{w s} \underset{w s}{2}+e^{2}\left(12 \cot 2 \theta_{W}\right) \mathscr{C}_{e Z} \\
& +\left(2 \sin \theta_{W} \cos \theta_{W}\right)\left[Y_{e}^{\dagger} Y_{e}\right]_{r w} \mathscr{C}_{e Z}-\cot \theta_{W}\left[Y_{e}^{\dagger}\right]_{r s}\left(C_{H W B}+i C_{H \widetilde{W} B}\right) \\
& +\frac{8}{3} e^{2}\left[Y_{e}^{\dagger}\right]_{r s}\left(\mathscr{C}_{\gamma \gamma}+i \tilde{\mathscr{C}}_{\gamma \gamma}\right)+e^{2}\left(\cot \theta_{W}-\frac{5}{3} \tan \theta_{W}\right)\left[Y_{e}^{\dagger}\right]_{r s}\left(\mathscr{C}_{\gamma Z}+i \widetilde{\mathscr{C}}_{\gamma Z}\right) \\
& +15\left[Y_{u}\right]_{w v} C_{\text {lequ }}^{(3)} \text {. }
\end{aligned}
$$

The current experimental limit [94] on $\operatorname{BR}(\mu \rightarrow e \gamma)$ is $5.7 \times 10^{-13}$ from the MEG experiment, which implies

$$
\frac{v}{\sqrt{2} m_{e}} \underset{\mu e}{\mathscr{C}_{e \gamma}} \lesssim 2.7 \times 10^{-4} \mathrm{TeV}^{-2}
$$

at the low energy scale $\mu \sim m_{\mu}$.

The lepton Yukawa couplings are diagonal in the mass eigenstate basis, so the $\mu \rightarrow e \gamma$ transition amplitude depends on $\mathscr{C}_{e \gamma}, \mathscr{C}_{e Z}$ and $C_{\text {lequ }}^{(3)}$. The bound eq. (5.65) implies

$$
\frac{m_{t}}{m_{e}} C_{\text {lequ }}^{(3)} \lesssim 1.4 \times 10^{-3} \mathrm{TeV}^{-2}
$$

using the estimate $\ln \left(\Lambda / m_{H}\right) /\left(16 \pi^{2}\right) \sim 0.01$ for the renormalization group evolution, and assuming that this term is the only contribution to $\mathscr{C}_{e \gamma}$ at low energies.

The anomalous magnetic moment of the muon is

$$
\delta a_{\mu}=-\frac{4 m_{\mu} v}{\sqrt{2}} \operatorname{Re} \mathscr{C}_{\mu \mu}
$$

which yields the limits

$$
\left|C_{H W B}\right| \lesssim 0.6 \mathrm{TeV}^{-2}, \quad\left|\mathscr{C}_{\gamma \gamma}\right| \lesssim 4 \mathrm{TeV}^{-2}, \quad\left|\frac{m_{t}}{m_{\mu}} \operatorname{Re} C_{l e q u}^{(3)}\right| \lesssim 7 \mathrm{TeV}^{-2}
$$

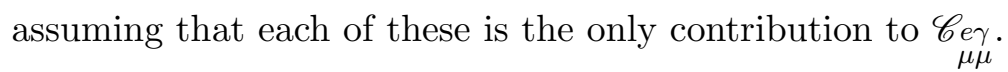


The bound on the electric dipole moment of the electron translates to the limits

$$
\left|C_{H \widetilde{W} B}\right| \lesssim 2 \times 10^{-3} \mathrm{TeV}^{-2}, \quad\left|\widetilde{\mathscr{C}}_{\gamma \gamma}\right| \lesssim 2 \times 10^{-2} \mathrm{TeV}^{-2}, \quad\left|\frac{m_{t}}{m_{e}} \operatorname{Im} C_{\text {lequ }}\right| \lesssim 3 \times 10^{-4} \mathrm{TeV}^{-2},
$$

using the recently measured upper bound [95], $d_{e}<1.05 \times 10^{-27} e \mathrm{~cm}$ from the ACME collaboration, again assuming each of these terms is the only contribution.

\section{Conclusions}

This paper completes the full calculation of the one-loop renormalization of the dimensionsix Lagrangian of the SM EFT. We present all of the remaining gauge terms in the $59 \times 59$ anomalous dimension matrix.

Many of the results are lengthy, but a few important cases such as $g g \rightarrow h, h \rightarrow \gamma \gamma$ and $h \rightarrow \gamma Z$ have simple RG equations which are given explicitly in this paper. We have computed the modification of the Higgs mass, self-interactions, and couplings to fermions and gauge bosons from $\mathcal{L}^{(6)}$. The dimension-six terms change the relation between the Higgs vacuum expectation value and $G_{F}$, and also contribute to the $\rho$ parameter. The RGE improvement of all of these relations is now known, and will be useful for future precision studies of the SM EFT. A complete analysis of the SM EFT is a formidable task, because $\mathcal{L}^{(6)}$ has 2499 independent parameters.

We have also discussed how the SM EFT provides a model-independent way to test the MFV hypothesis, and how the full SM EFT RGE mixes flavor violation between the different operator sectors. A few applications of our results have been given in this paper.

\section{Acknowledgments}

This work was supported in part by DOE grant DE-SC0009919. MT thanks W. Skiba for helpful conversations. RA and AM thank B. Shotwell, D. Stone, H.-M. Chang and C. Murphy for useful discussions. We would also like to thank C. Zhang for pointing out a typo.

\section{A Flavor representations and parameter counting}

In this appendix, we briefly discuss the flavor representations of the operators, and the parameter counting of table 2 .

Operators in classes 1-4 have no flavor indices, and the counting is trivial.

Class 5 and 6 operator coefficients are $n_{g} \times n_{g}$ complex matrices $M_{r s}$ in flavor space, with $n_{g}^{2}$ complex entries. The real matrix elements give the $n_{g}^{2} C P$-even parmeters and the imaginary matrix elements yield $n_{g}^{2} C P$-odd entries.

Class 7 operators, other than $Q_{H u d}$ are hermitian, so their coefficients are $n_{g} \times n_{g}$ hermitian matrices $H_{r s}$ in flavor space, which can be written as $H_{r s}=S_{r s}+i A_{r s}$, where $S$ is real-symmetric and $C P$-even with $n_{e}=n_{g}\left(n_{g}+1\right) / 2$ parameters, and $A$ is realantisymmetric and $C P$-odd, with $n_{o}=n_{g}\left(n_{g}-1\right) / 2$ parameters. $Q_{H u d}$, which is not hermitian, is an $n_{g} \times n_{g}$ complex matrix with $n_{g}^{2} C P$-even and $n_{g}^{2} C P$-odd parameters. 


\begin{tabular}{|c|c|c|c|c|c|c|c|}
\hline \multicolumn{2}{|r|}{$1: X^{3}$} & & & & & \multirow{2}{*}{\multicolumn{2}{|c|}{$5: \psi^{2} H^{3}+$ h.c. }} \\
\hline \multirow{3}{*}{$\begin{array}{c}Q_{G} \\
Q_{\widetilde{G}} \\
Q_{W}\end{array}$} & \multirow{3}{*}{$\begin{array}{l}f^{A B C} G_{\mu}^{A \nu} G_{\nu}^{B \rho} G_{\rho}^{C \mu} \\
f^{A B C} \widetilde{G}_{\mu}^{A \nu} G_{\nu}^{B \rho} G_{\rho}^{C \mu} \\
\epsilon^{I J K} W_{\mu}^{I \nu} W_{\nu}^{J \rho} W_{\rho}^{K \mu}\end{array}$} & \multirow{2}{*}{\multicolumn{2}{|c|}{$2: H^{6}$}} & \multicolumn{2}{|r|}{$3: H^{4} D^{2}$} & & \\
\hline & & & & & & \multirow{3}{*}{$\begin{array}{l}Q_{e H} \\
Q_{u H} \\
Q_{d H}\end{array}$} & \multirow{3}{*}{$\begin{array}{l}\left(H^{\dagger} H\right)\left(\bar{l}_{p} e_{r} H\right) \\
\left(H^{\dagger} H\right)\left(\bar{q}_{p} u_{r} \widetilde{H}\right) \\
\left(H^{\dagger} H\right)\left(\bar{q}_{p} d_{r} H\right)\end{array}$} \\
\hline & & $Q_{H}$ & $\left(H^{\dagger} H\right)^{3}$ & $\begin{array}{l}Q_{H \square} \\
O_{H D}\end{array}$ & $\begin{array}{c}\left(H^{\top} H\right) \square\left(H^{\top} H\right) \\
\left(H^{\dagger} D H\right)^{*}\left(H^{\dagger} D H\right)\end{array}$ & & \\
\hline $\begin{array}{l}Q W \\
Q_{\overparen{W}}\end{array}$ & $\epsilon^{I J K} \widetilde{W}_{\mu}^{I \nu} W_{\nu}^{J \rho} W_{\rho}^{K \mu}$ & & & & & & \\
\hline
\end{tabular}

\begin{tabular}{|c|c||c|c||c|c|}
\hline \multicolumn{2}{|c|}{$4: X^{2} H^{2}$} & \multicolumn{2}{|c|}{$6: \psi^{2} X H+$ h.c. } & \multicolumn{2}{|c|}{$7: \psi^{2} H^{2} D$} \\
\hline$Q_{H G}$ & $H^{\dagger} H G_{\mu \nu}^{A} G^{A \mu \nu}$ & $Q_{e W}$ & $\left(\bar{l}_{p} \sigma^{\mu \nu} e_{r}\right) \tau^{I} H W_{\mu \nu}^{I}$ & $Q_{H l}^{(1)}$ & $\left(H^{\dagger} i \overleftrightarrow{D}_{\mu} H\right)\left(\bar{l}_{p} \gamma^{\mu} l_{r}\right)$ \\
$Q_{H \widetilde{G}}$ & $H^{\dagger} H \widetilde{G}_{\mu \nu}^{A} G^{A \mu \nu}$ & $Q_{e B}$ & $\left(\bar{l}_{p} \sigma^{\mu \nu} e_{r}\right) H B_{\mu \nu}$ & $Q_{H l}^{(3)}$ & $\left(H^{\dagger} i \overleftrightarrow{D}_{\mu}^{I} H\right)\left(\bar{l}_{p} \tau^{I} \gamma^{\mu} l_{r}\right)$ \\
$Q_{H W}$ & $H^{\dagger} H W_{\mu \nu}^{I} W^{I \mu \nu}$ & $Q_{u G}$ & $\left(\bar{q}_{p} \sigma^{\mu \nu} T^{A} u_{r}\right) \widetilde{H} G_{\mu \nu}^{A}$ & $Q_{H e}$ & $\left(H^{\dagger} i \overleftrightarrow{D}_{\mu} H\right)\left(\bar{e}_{p} \gamma^{\mu} e_{r}\right)$ \\
$Q_{H \widetilde{W}}$ & $H^{\dagger} H \widetilde{W}_{\mu \nu}^{I} W^{I \mu \nu}$ & $Q_{u W}$ & $\left(\bar{q}_{p} \sigma^{\mu \nu} u_{r}\right) \tau^{I} \widetilde{H} W_{\mu \nu}^{I}$ & $Q_{H q}^{(1)}$ & $\left(H^{\dagger} i \overleftrightarrow{D}_{\mu} H\right)\left(\bar{q}_{p} \gamma^{\mu} q_{r}\right)$ \\
$Q_{H B}$ & $H^{\dagger} H B_{\mu \nu} B^{\mu \nu}$ & $Q_{u B}$ & $\left(\bar{q}_{p} \sigma^{\mu \nu} u_{r}\right) \widetilde{H} B_{\mu \nu}$ & $Q_{H q}^{(3)}$ & $\left(H^{\dagger} i \overleftrightarrow{D}_{\mu}^{I} H\right)\left(\bar{q}_{p} \tau^{I} \gamma^{\mu} q_{r}\right)$ \\
$Q_{H \widetilde{B}}$ & $H^{\dagger} H \widetilde{B}_{\mu \nu} B^{\mu \nu}$ & $Q_{d G}$ & $\left(\bar{q}_{p} \sigma^{\mu \nu} T^{A} d_{r}\right) H G_{\mu \nu}^{A}$ & $Q_{H u}$ & $\left(H^{\dagger} i \overleftrightarrow{D}_{\mu} H\right)\left(\bar{u}_{p} \gamma^{\mu} u_{r}\right)$ \\
$Q_{H W B}$ & $H^{\dagger} \tau^{I} H W_{\mu \nu}^{I} B^{\mu \nu}$ & $Q_{d W}$ & $\left(\bar{q}_{p} \sigma^{\mu \nu} d_{r}\right) \tau^{I} H W_{\mu \nu}^{I}$ & $Q_{H d}$ & $\left(H^{\dagger} i \overleftrightarrow{D}_{\mu} H\right)\left(\bar{d}_{p} \gamma^{\mu} d_{r}\right)$ \\
$Q_{H \widetilde{W} B}$ & $H^{\dagger} \tau^{I} H \widetilde{W}_{\mu \nu}^{I} B^{\mu \nu}$ & $Q_{d B}$ & $\left(\bar{q}_{p} \sigma^{\mu \nu} d_{r}\right) H B_{\mu \nu}$ & $Q_{H u d}+$ h.c. & $i\left(\widetilde{H}^{\dagger} D_{\mu} H\right)\left(\bar{u}_{p} \gamma^{\mu} d_{r}\right)$ \\
\hline
\end{tabular}

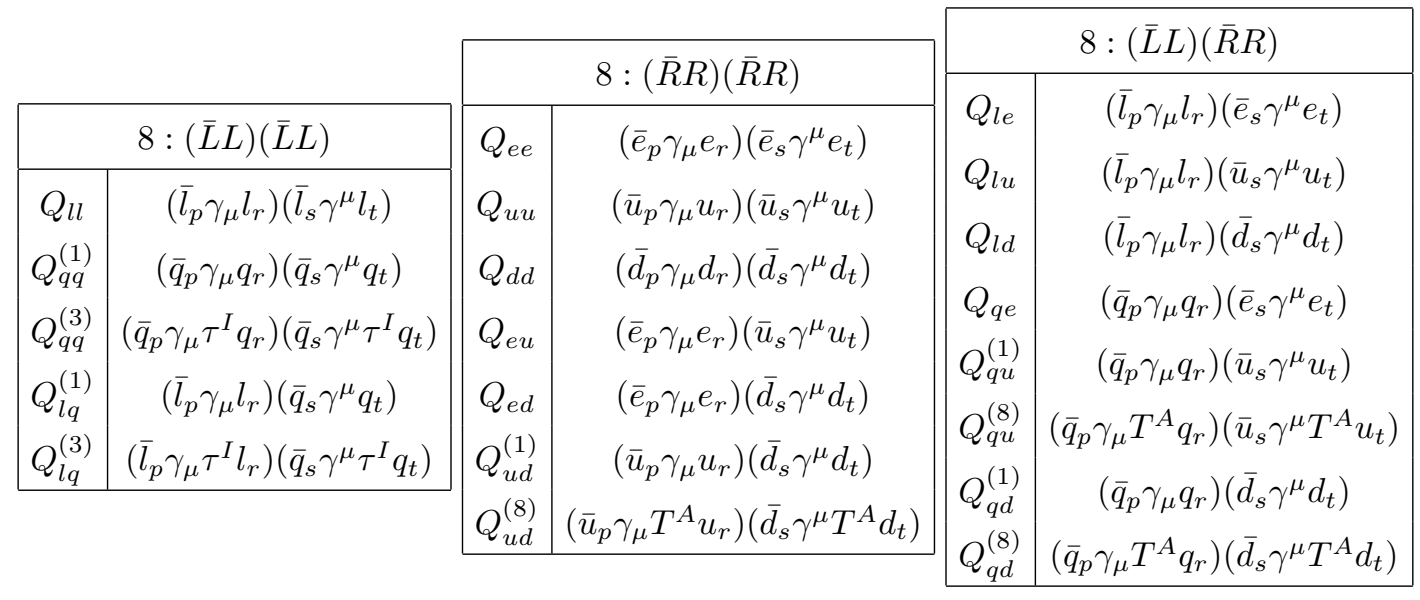

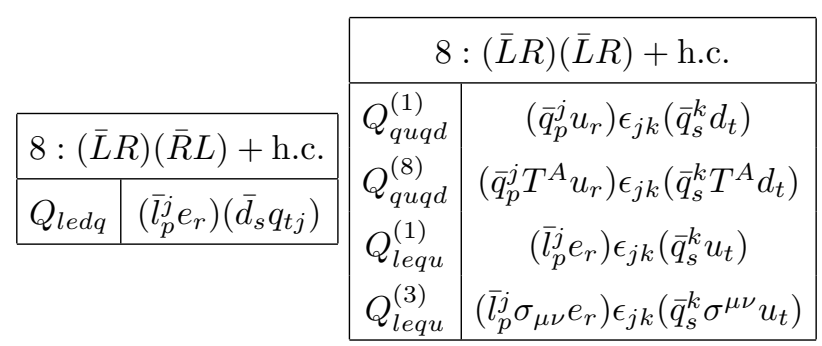

Table 1. The 59 independent dimension-six operators built from Standard Model fields which conserve baryon number, as given in ref. [9]. The operators are divided into eight classes: $X^{3}, H^{6}$, etc. Operators with + h.c. in the table heading also have hermitian conjugates, as does the $\psi^{2} H^{2} D$ operator $Q_{H u d}$. The subscripts $p, r, s, t$ are flavor indices. 


\begin{tabular}{|c|c|c|c|c|c|c|c|}
\hline \multirow[t]{2}{*}{ Class } & \multirow[t]{2}{*}{$N_{\mathrm{op}}$} & \multicolumn{3}{|l|}{$C P$-even } & \multicolumn{3}{|l|}{$C P$-odd } \\
\hline & & $n_{g}$ & 1 & 3 & $n_{g}$ & 1 & 3 \\
\hline 1 & 4 & 2 & 2 & 2 & 2 & 2 & 2 \\
\hline 2 & 1 & 1 & 1 & 1 & 0 & 0 & 0 \\
\hline 3 & 2 & 2 & 2 & 2 & 0 & 0 & 0 \\
\hline 4 & 8 & 4 & 4 & 4 & 4 & 4 & 4 \\
\hline 5 & 3 & $3 n_{g}^{2}$ & 3 & 27 & $3 n_{g}^{2}$ & 3 & 27 \\
\hline 6 & 8 & $8 n_{g}^{2}$ & 8 & 72 & $8 n_{g}^{2}$ & 8 & 72 \\
\hline 7 & 8 & $\frac{1}{2} n_{g}\left(9 n_{g}+7\right)$ & 8 & 51 & $\frac{1}{2} n_{g}\left(9 n_{g}-7\right)$ & 1 & 30 \\
\hline $8:(\bar{L} L)(\bar{L} L)$ & 5 & $\frac{1}{4} n_{g}^{2}\left(7 n_{g}^{2}+13\right)$ & 5 & 171 & $\frac{7}{4} n_{g}^{2}\left(n_{g}-1\right)\left(n_{g}+1\right)$ & 0 & 126 \\
\hline $8:(\bar{R} R)(\bar{R} R)$ & 7 & $\frac{1}{8} n_{g}\left(21 n_{g}^{3}+2 n_{g}^{2}+31 n_{g}+2\right)$ & 7 & 255 & $\frac{1}{8} n_{g}\left(21 n_{g}+2\right)\left(n_{g}-1\right)\left(n_{g}+1\right)$ & 0 & 195 \\
\hline $8:(\bar{L} L)(\bar{R} R)$ & 8 & $4 n_{g}^{2}\left(n_{g}^{2}+1\right)$ & 8 & 360 & $4 n_{g}^{2}\left(n_{g}-1\right)\left(n_{g}+1\right)$ & 0 & 288 \\
\hline $8:(\bar{L} R)(\bar{R} L)$ & 1 & $n_{g}^{4}$ & 1 & 81 & $n_{g}^{4}$ & 1 & 81 \\
\hline $8:(\bar{L} R)(\bar{L} R)$ & 4 & $4 n_{g}^{4}$ & 4 & 324 & $4 n_{g}^{4}$ & 4 & 324 \\
\hline 8: All & 25 & $\frac{1}{8} n_{g}\left(107 n_{g}^{3}+2 n_{g}^{2}+89 n_{g}+2\right)$ & & 1191 & $\frac{1}{8} n_{g}\left(107 n_{g}^{3}+2 n_{g}^{2}-67 n_{g}-2\right)$ & & 1014 \\
\hline Total & 59 & $\frac{1}{8}\left(107 n_{g}^{4}+2 n_{g}^{3}+213 n_{g}^{2}+30 n_{g}+72\right)$ & 53 & 1350 & $\frac{1}{8}\left(107 n_{g}^{4}+2 n_{g}^{3}+57 n_{g}^{2}-30 n_{g}+48\right)$ & 23 & 1149 \\
\hline
\end{tabular}

Table 2. Number of $C P$-even and $C P$-odd coefficients in $\mathcal{L}^{(6)}$ for $n_{g}$ flavors. The total number of coefficients is $\left(107 n_{g}^{4}+2 n_{g}^{3}+135 n_{g}^{2}+60\right) / 4$, which is 76 for $n_{g}=1$ and 2499 for $n_{g}=3$.

\begin{tabular}{|c|c|c|c|c|c|c|c|c|}
\hline & $H^{6}$ & $H^{4} D^{2}$ & $y \psi^{2} H^{3}$ & $\psi^{2} H^{2} D$ & $\psi^{4}$ & $g^{2} X^{2} H^{2}$ & $g y \psi^{2} X H$ & $g^{3} X^{3}$ \\
\hline Class & 2 & 3 & 5 & 7 & 8 & 4 & 6 & 1 \\
\hline NDA Weight & 2 & 1 & 1 & 1 & 1 & 0 & 0 & -1 \\
\hline$H^{6}$ & $\lambda, y^{2}, g^{2}$ & $\lambda^{2}, \lambda g^{2}, g^{4}$ & $\lambda y^{2}, y^{4}$ & $\lambda y^{2}, \lambda g^{2}, y_{y}^{\prime}$ & 0 & $\lambda g^{4}, g^{6}$ & 0 & $X \phi \phi$ \\
\hline$H^{4} D^{2}$ & 0 & $\lambda, y^{2}, g^{2}$ & $\left|y^{2}\right|$ & $y^{2}, g^{2}$ & 0 & $g^{A} /$ & $\left.\not\right|^{2} \mid g f^{2}$ & $\operatorname{gos}^{6} /$ \\
\hline$y \psi^{2} H^{3}$ & 0 & $\lambda, y^{2}, g^{2}$ & $\lambda, y^{2}, g^{2}$ & $\lambda, y^{2}, g^{2}$ & $\lambda, y^{2}$ & $g^{4}$ & $g^{2} / X, g^{4}, g^{2} y^{2}$ & $g^{6} /$ \\
\hline$\psi^{2} H^{2} D$ & 0 & $g^{2}, y^{2}$ & $\left|y^{2}\right|$ & $g^{2}, X, y^{2}$ & $g^{2}, y^{2}$ & $\left.g\right|^{A} /$ & $\not g^{2} /\left.y\right|^{2}$ & $g^{6} /$ \\
\hline$\psi^{4}$ & 0 & 0 & 0 & $g^{2}, y^{2}$ & $g^{2}, y^{2}$ & 0 & $g^{2} y^{2}$ & $\not g^{6} /$ \\
\hline$g^{2} X^{2} H^{2}$ & 0 & 1 & 0 & 1 & 0 & $\lambda, y^{2}, g^{2}$ & $y^{2}$ & $g^{4}$ \\
\hline$g y \psi^{2} X H$ & 0 & 0 & 1 & 1 & 1 & $g^{2}$ & $g^{2}, y^{2}$ & $g^{4}$ \\
\hline$g^{3} X^{3}$ & 0 & 0 & 0 & 0 & 0 & 1 & 0 & $g^{2}$ \\
\hline
\end{tabular}

Table 3. Form of the one-loop anomalous dimension matrix $\widehat{\gamma}_{i j}$ for dimension-six operators $\widehat{Q}_{i}$ rescaled according to naive dimensional analysis. The operators are ordered by NDA weight, rather than by operator class. The possible entries allowed by the one-loop Feynman graphs are shown. The cross-hatched entries vanish.

The four-fermion operators in Class 8 are the only non-trivial case. The $(\bar{L} R)(\bar{R} L)$ and $(\bar{L} R)(\bar{L} R)$ operators are not hermitian, and each has $n_{g}^{4} C P$-even and $n_{g}^{4} C P$-odd parameters, since the operator has 4 independent flavor indices. The $(\bar{L} L)(\bar{R} R)$ operators 
are the product of $L$ and $R$ currents, each of which has $n_{e} C P$-even and $n_{o} C P$-odd components, for $n_{e}^{2}+n_{o}^{2} C P$-even and $2 n_{e} n_{o} C P$-odd terms. The counting for $(\bar{L} L)(\bar{L} L)$ and $(\bar{R} R)(\bar{R} R)$ operators when the currents are different, $Q_{l q}^{(1,3)}, Q_{e u}, Q_{e d}, Q_{u d}^{(1,3)}$, is the same as for the $(\bar{L} L)(\bar{R} R)$ operators. The interesting case is for $Q_{l l}, Q_{q q}^{(1,3)}, Q_{u u}, Q_{d d}$ where the two currents are identical, so that all four flavor indices transform under the same $\mathrm{SU}\left(n_{g}\right)$ flavor group. The operators transform as the $\mathbf{1}+\operatorname{adj}+\operatorname{adj}+\bar{a} a+\bar{s} s$ where adj is the adjoint representation, $\bar{a} a$ is the representation $T_{[k l]}^{[i j]}$ antisymmetric in the upper and lower indices, and $\bar{s} s$ is the representation $T_{(k l)}^{(i j)}$ symmetric in the upper and lower indices. ${ }^{9}$ The $\bar{a} a$ representation vanishes for $n_{g}=3$. The singlet has one $C P$-even parameter, the adjoint has $\left(n_{g}-1\right)\left(n_{g}+2\right) / 2 C P$-even and $n_{g}\left(n_{g}-1\right) / 2 C P$-odd parameters, $\bar{a} a$ has $n_{g}\left(n_{g}-3\right)\left(n_{g}^{2}+n_{g}+2\right) / 8 C P$-even and $n_{g}\left(n_{g}-3\right)\left(n_{g}-1\right)\left(n_{g}+2\right) / 8 C P$-odd parameters, and $\bar{s} s$ has $n_{g}\left(n_{g}-1\right)\left(n_{g}+1\right)\left(n_{g}+2\right) / 8 C P$-even and $n_{g}\left(n_{g}-1\right)\left(n_{g}^{2}+3 n_{g}-2\right) / 8 C P$-odd parameters. The operator $Q_{e e}$ is a special case, because of the Fierz identity

$$
\left(\bar{e}_{p} \gamma_{\mu} e_{r}\right)\left(\bar{e}_{s} \gamma_{\mu} e_{t}\right)=\left(\bar{e}_{s} \gamma_{\mu} e_{r}\right)\left(\bar{e}_{p} \gamma_{\mu} e_{t}\right)
$$

which implies that the operator must be symmetric in the two $e$ indices and in the two $\bar{e}$ indices. This identity does not hold for the other fermions, because they have $\mathrm{SU}(2)$ or color indices. $Q_{e e}$ transforms as $1+\operatorname{adj}+\bar{s} s$ because of the Fierz identity.

Adding up the individual contributions gives table 2 .

\section{B Conversion of $\boldsymbol{P}_{i}$ operators to the standard basis}

The equations of motion can be used to express the operators $P_{i}$ in the standard basis. The identifications are

$$
\begin{aligned}
& \mathcal{P}_{B}=\frac{1}{2} \mathrm{y}_{H} g_{1}^{2} Q_{H \square}+2 g_{1}^{2} \mathrm{y}_{H} Q_{H D}+\frac{1}{2} g_{1}^{2}\left[\underset{t t}{\mathrm{y}_{l} Q_{H l}^{(1)}}+\underset{t t}{\mathrm{y}_{e} Q_{H e}}+\underset{t t}{\mathrm{y}_{q}} Q_{H q}^{(1)}+\mathrm{y}_{u} Q_{t t}+\mathrm{y}_{d} Q_{t t}\right], \\
& \mathcal{P}_{W}=\frac{3}{4} g_{2}^{2} Q_{H \square}-\frac{1}{2} g_{2}^{2} m_{H}^{2}\left(H^{\dagger} H\right)^{2}+2 g_{2}^{2} \lambda Q_{H}+\frac{1}{4} g_{2}^{2}\left[\begin{array}{c}
Q_{t t}^{(3)} \\
t t
\end{array} Q_{t t}^{(3)}\right] \\
& +\frac{1}{2} g_{2}^{2}\left(\left[Y_{u}^{\dagger}\right]_{r s} Q_{r s}+\left[Y_{d}^{\dagger}\right]_{r s} Q_{r s}+\left[Y_{e}^{\dagger}\right]_{r s} Q_{r s}+\text { h.c. }\right), \\
& \mathcal{P}_{H B}=\frac{1}{2} g_{1}^{2} \mathrm{y}_{H} Q_{H \square}+2 g_{1}^{2} \mathrm{y}_{H} Q_{H D}-\frac{1}{2} \mathrm{y}_{H} g_{1}^{2} Q_{H B}-\frac{1}{4} g_{1} g_{2} Q_{H W B}, \\
& +\frac{1}{2} g_{1}^{2}\left[\mathrm{y}_{l} Q_{t t}^{(1)}+\mathrm{y}_{e} Q_{t t}^{H e}+\mathrm{y}_{q} Q_{H q}^{(1)}+\mathrm{y}_{u} Q_{t t}{ }_{t t}+\mathrm{y}_{d} Q_{t t}{ }_{t t}\right], \\
& \mathcal{P}_{H W}=\frac{3}{4} g_{2}^{2} Q_{H \square}-\frac{1}{2} g_{2}^{2} m_{H}^{2}\left(H^{\dagger} H\right)^{2}+2 g_{2}^{2} \lambda Q_{H}-\frac{1}{4} g_{2}^{2} Q_{H W}-\frac{1}{2} \mathrm{y}_{H} g_{1} g_{2} Q_{H W B}+\frac{1}{4} g_{2}^{2}\left[Q_{H l}^{(3)}+Q_{H q}^{(3)}\right] \\
& +\frac{1}{2} g_{2}^{2}\left(\left[Y_{u}^{\dagger}\right]_{r s} Q_{r s}+\left[Y_{d}^{\dagger}\right]_{r s} Q_{r s}+\underset{r s}{\left[Y_{e}^{\dagger}\right]_{r s} Q_{e H}}+\text { h.c. }\right), \\
& P_{T}=-Q_{H \square}-4 Q_{H D} .
\end{aligned}
$$

\footnotetext{
${ }^{9}$ The relevant group theory results can be found, for example, in refs. [96, 97].
} 


\section{Results}

The renormalization group equations by operator class are given below. The complete RG equations for the dimension-six operators are given by adding eqs. (6.1)-(6.4) of ref. [12], the equations in the appendices of ref. [10] and the equations given below. Eqs. (4.3)-(4.5) of ref. [12] give the renormalization group evolution of SM couplings due to dimension-six operators.

The parameters $\eta_{1-5}$ are defined in the appendix of ref. [10]. Some equations use $\xi_{B}$, defined by

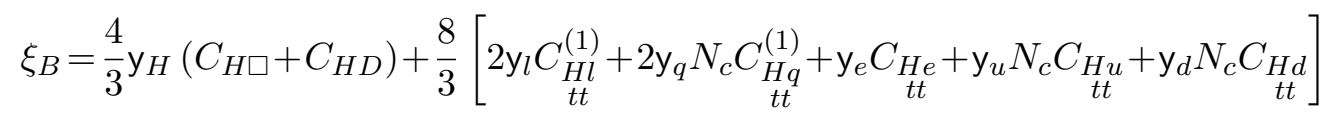

The other parameters are $c_{A, 2}=2, c_{F, 2}=3 / 4, c_{A, 3}=N_{c}, c_{F, 3}=\left(N_{c}^{2}-1\right) /\left(2 N_{c}\right)$ with $N_{c}=3, b_{0,1}=-1 / 6-20 n_{g} / 9, b_{0,2}=43 / 6-4 n_{g} / 3$ and $b_{0,3}=11-4 n_{g} / 3$.

\section{C.1 $X^{3}$}

$$
\begin{aligned}
\dot{C}_{G} & =\left(12 c_{A, 3}-3 b_{0,3}\right) g_{3}^{2} C_{G} & \dot{C}_{\widetilde{G}} & =\left(12 c_{A, 3}-3 b_{0,3}\right) g_{3}^{2} C_{\widetilde{G}} \\
\dot{C}_{W} & =\left(12 c_{A, 2}-3 b_{0,2}\right) g_{2}^{2} C_{W} & \dot{C}_{\widetilde{W}} & =\left(12 c_{A, 2}-3 b_{0,2}\right) g_{2}^{2} C_{\widetilde{W}}
\end{aligned}
$$

\section{C.2 $H^{6}$}

$$
\begin{aligned}
\dot{C}_{H}= & \left(-\frac{27}{2} g_{2}^{2}-\frac{9}{2} g_{1}^{2}\right) C_{H}+\lambda\left[\frac{40}{3} g_{2}^{2} C_{H \square}+\left(-6 g_{2}^{2}+24 g_{1}^{2} \mathrm{y}_{H}^{2}\right) C_{H D}\right]-\frac{3}{4}\left(4 \mathrm{y}_{H}^{2} g_{1}^{2}+g_{2}^{2}\right)^{2} C_{H D} \\
& +12 \lambda\left(3 g_{2}^{2} C_{H W}+4 g_{1}^{2} \mathrm{y}_{H}^{2} C_{H B}+2 g_{1} g_{2} \mathrm{y}_{H} C_{H W B}\right)-\left(12 g_{1}^{2} g_{2}^{2} \mathrm{y}_{H}^{2}+9 g_{2}^{4}\right) C_{H W} \\
& -\left(48 g_{1}^{4} \mathrm{y}_{H}^{4}+12 g_{1}^{2} g_{2}^{2} \mathrm{y}_{H}^{2}\right) C_{H B}-\left(24 g_{1}^{3} g_{2} \mathrm{y}_{H}^{3}+6 g_{1} g_{2}^{3} \mathrm{y}_{H}\right) C_{H W B}+\frac{16}{3} \lambda g_{2}^{2}\left(C_{H l}^{(3)}+N_{c} C_{H q}^{(3)}\right)
\end{aligned}
$$

\section{C.3 $H^{4} D^{2}$}

$$
\begin{aligned}
& \dot{C}_{H \square}=-\left(4 g_{2}^{2}+\frac{16}{3} g_{1}^{2} y_{H}^{2}\right) C_{H \square}+\frac{20}{3} g_{1}^{2} y_{H}^{2} C_{H D}+2 g_{2}^{2}\left(C_{t t}^{(3)}+N_{c} C_{t q}^{(3)}\right) \\
& +\frac{4}{3} g_{1}^{2} \mathrm{y}_{H}\left(N_{c} \mathrm{y}_{u} C_{t u}+N_{c t} \mathrm{y}_{d} C_{H d}+\mathrm{y}_{e} C_{t e}+2 N_{c t} \mathrm{y}_{q} C_{t t}^{(1)}+2 \mathrm{y}_{l} C_{t t}^{(1)}\right) \\
& \dot{C}_{H D}=\frac{80}{3} g_{1}^{2} \mathrm{y}_{H}^{2} C_{H \square}+\left(\frac{9}{2} g_{2}^{2}-\frac{10}{3} g_{1}^{2} \mathrm{y}_{H}^{2}\right) C_{H D} \\
& +\frac{16}{3} g_{1}^{2} \mathrm{y}_{H}\left(N_{c} \mathrm{y}_{u} C_{H_{u} u}+N_{c} \mathrm{y}_{d} C_{t t}+\mathrm{y}_{e} C_{t t}+2 N_{c t} \mathrm{y}_{q} C_{t t}^{(1)}+2 \mathrm{y}_{l} C_{H l}^{(1)}\right)
\end{aligned}
$$




\section{C.4 $X^{2} H^{2}$}

$$
\begin{aligned}
\dot{C}_{H G} & =\left(-6 \mathrm{y}_{H}^{2} g_{1}^{2}-\frac{9}{2} g_{2}^{2}-2 b_{0,3} g_{3}^{2}\right) C_{H G} \\
\dot{C}_{H B} & =\left(2 \mathrm{y}_{H}^{2} g_{1}^{2}-\frac{9}{2} g_{2}^{2}-2 b_{0,1} g_{1}^{2}\right) C_{H B}+6 g_{1} g_{2} \mathrm{y}_{H} C_{H W B} \\
\dot{C}_{H W} & =-15 g_{2}^{3} C_{W}+\left(-6 \mathrm{y}_{H}^{2} g_{1}^{2}-\frac{5}{2} g_{2}^{2}-2 b_{0,2} g_{2}^{2}\right) C_{H W}+2 g_{1} g_{2} \mathrm{y}_{H} C_{H W B} \\
\dot{C}_{H W B} & =6 g_{1} g_{2}^{2} \mathrm{y}_{H} C_{W}+\left(-2 \mathrm{y}_{H}^{2} g_{1}^{2}+\frac{9}{2} g_{2}^{2}-b_{0,1} g_{1}^{2}-b_{0,2} g_{2}^{2}\right) C_{H W B}+4 g_{1} g_{2} \mathrm{y}_{H} C_{H B}+4 g_{1} g_{2} \mathrm{y}_{H} C_{H W} \\
\dot{C}_{H \widetilde{G}} & =\left(-6 \mathrm{y}_{H}^{2} g_{1}^{2}-\frac{9}{2} g_{2}^{2}-2 b_{0,3} g_{3}^{2}\right) C_{H \widetilde{G}} \\
\dot{C}_{H \widetilde{B}} & =\left(2 \mathrm{y}_{H}^{2} g_{1}^{2}-\frac{9}{2} g_{2}^{2}-2 b_{0,1} g_{1}^{2}\right) C_{H \widetilde{B}}+6 g_{1} g_{2} \mathrm{y}_{H} C_{H \widetilde{W} B} \\
\dot{C}_{H \widetilde{W}} & =-15 g_{2}^{3} C_{\widetilde{W}}+\left(-6 \mathrm{y}_{H}^{2} g_{1}^{2}-\frac{5}{2} g_{2}^{2}-2 b_{0,2} g_{2}^{2}\right) C_{H \widetilde{W}}+2 g_{1} g_{2} \mathrm{y}_{H} C_{H \widetilde{W} B} \\
\dot{C}_{H \widetilde{W} B} & =6 g_{1} g_{2}^{2} \mathrm{y}_{H} C_{\widetilde{W}}+\left(-2 \mathrm{y}_{H}^{2} g_{1}^{2}+\frac{9}{2} g_{2}^{2}-b_{0,1} g_{1}^{2}-b_{0,2} g_{2}^{2}\right) C_{H \widetilde{W} B}+4 g_{1} g_{2} \mathrm{y}_{H} C_{H \widetilde{B}}+4 g_{1} g_{2} \mathrm{y}_{H} C_{H \widetilde{W}}
\end{aligned}
$$

\section{C.5 $\psi^{2} H^{3}$}

$$
\begin{aligned}
& \dot{C}_{r s}=\left[Y_{e}^{\dagger}\right]_{r s}\left[\frac{10}{3} g_{2}^{2} C_{H \square}+\left(-\frac{3}{2} g_{2}^{2}+6 g_{1}^{2} \mathrm{y}_{H}^{2}\right) C_{H D}\right]-\left[3\left(3 \mathrm{y}_{l}^{2}+3 \mathrm{y}_{e}^{2}-4 y_{l} \mathrm{y}_{e}\right) g_{1}^{2}+\frac{27}{4} g_{2}^{2}\right] C_{e H} \\
& +3\left[Y_{e}^{\dagger}\right]_{r s}\left(3 g_{2}^{2} C_{H W}+4\left(\mathrm{y}_{H}^{2}+2 \mathrm{y}_{l} \mathrm{y}_{e}\right) g_{1}^{2} C_{H B}+2 g_{1} g_{2} \mathrm{y}_{l} C_{H W B}\right)-3\left(3 g_{1} \mathrm{y}_{e} C_{e B}+g_{2} C_{e W}\right)\left[Y_{e} Y_{e}^{\dagger}\right]_{t s} \\
& -3\left[Y_{e}^{\dagger} Y_{e}\right]_{r v}\left(2 g_{1}\left(\mathrm{y}_{l}+\mathrm{y}_{e}\right) C_{e B}-g_{2} C_{e W}\right)-6\left(4 g_{1}^{3} \mathrm{y}_{H}^{2} \mathrm{y}_{e}+4 g_{1}^{3} \mathrm{y}_{H}^{2} \mathrm{y}_{l}+g_{2}^{2} g_{1} \mathrm{y}_{H}\right) C_{r s} \\
& -3\left(4 g_{1}^{2} g_{2} \mathrm{y}_{H} \mathrm{y}_{e}+4 g_{1}^{2} g_{2} \mathrm{y}_{H} \mathrm{y}_{l}+3 g_{2}^{3}\right) C_{e W}+\left(3 g_{2}^{2}+12 g_{1}^{2} \mathrm{y}_{l} \mathrm{y}_{H}\right)\left[Y_{e}^{\dagger}\right]_{r t} C_{H_{s}} \\
& +12 g_{1}^{2} \mathrm{y}_{e} \mathrm{y}_{H} C_{r t}^{(1)}\left[Y_{e}^{\dagger}\right]_{t s}+12 g_{1}^{2} \mathrm{y}_{e} \mathrm{y}_{H} C_{r t}^{(3)}\left[Y_{d}^{\dagger}\right]_{t s}+\frac{4}{3} g_{2}^{2}\left[Y_{e}^{\dagger}\right]_{r s}\left(\underset{t t}{C_{t l}^{(3)}}+N_{c} C_{t t}^{(3)}\right) \\
& \dot{C}_{r s}=\left[Y_{u}^{\dagger}\right]_{r s}\left[\frac{10}{3} g_{2}^{2} C_{H \square}+\left(-\frac{3}{2} g_{2}^{2}+6 g_{1}^{2} \mathrm{y}_{H}^{2}\right) C_{H D}\right]-\left[3\left(3 \mathrm{y}_{q}^{2}+3 \mathrm{y}_{u}^{2}-4 \mathrm{y}_{q} \mathrm{y}_{u}\right) g_{1}^{2}+\frac{27}{4} g_{2}^{2}+6 c_{F, 3} g_{3}^{2}\right] C_{r s} \\
& +3\left[Y_{u}^{\dagger}\right]_{r s}\left(8 g_{3}^{2} c_{F, 3} C_{H G}+3 g_{2}^{2} C_{H W}+4\left(\mathrm{y}_{H}^{2}+2 \mathrm{y}_{q} \mathrm{y}_{u}\right) g_{1}^{2} C_{H B}-2 \mathrm{y}_{q} g_{1} g_{2} C_{H W B}\right) \\
& -12\left[Y_{d}^{\dagger} Y_{d}\right]_{r t} g_{2} C_{u W}-6 g_{2} C_{d W}\left[Y_{d} Y_{u}^{\dagger}\right]_{t s}-3\left(4 g_{3} c_{F, 3} C_{u G}+g_{2 t} C_{u W}+\left(3 \mathrm{y}_{u}+\mathrm{y}_{d}\right) g_{1} C_{u B}\right)\left[Y_{u} Y_{u}^{\dagger}\right]_{t s} \\
& -3\left[Y_{u}^{\dagger} Y_{u}\right]_{r v}\left(4 c_{F, 3} g_{3} C_{u G}-g_{2} C_{u W}+2\left(\mathrm{y}_{q}+\mathrm{y}_{u}\right) g_{1} C_{u B}\right)-6\left(4 g_{1}^{3} \mathrm{y}_{H}^{2} \mathrm{y}_{u}+4 g_{1}^{3} \mathrm{y}_{H}^{2} \mathrm{y}_{q}-g_{2}^{2} g_{1} \mathrm{y}_{H}\right) C_{u B} \\
& +3\left(4 g_{1}^{2} g_{2} \mathrm{y}_{H} \mathrm{y}_{u}+4 g_{1}^{2} g_{2} \mathrm{y}_{H} \mathrm{y}_{q}-3 g_{2}^{3}\right) C_{r s}-\left(3 g_{2}^{2}-12 g_{1}^{2} \mathrm{y}_{q} \mathrm{y}_{H}\right)\left[Y_{u}^{\dagger}\right]_{r t} C_{H u} \\
& +3 g_{2}^{2}\left[Y_{d}^{\dagger}\right]_{r t} C_{H_{s t}}^{*}+12 g_{1}^{2} \mathrm{y}_{u} \mathrm{y}_{H} C_{r t}^{(1)}\left[Y_{u}^{\dagger}\right]_{t s}-12 g_{1}^{2} \mathrm{y}_{u} \mathrm{y}_{H} C_{r t}^{(3)}\left[Y_{u}^{\dagger}\right]_{t s}+\frac{4}{3} g_{2}^{2}\left[Y_{u}^{\dagger}\right]_{r s}\left(C_{t t}^{(3)}+N_{c} C_{t t}^{(3)}\right) \\
& \dot{C}_{d s}=\left[Y_{d}^{\dagger}\right]_{r s}\left[\frac{10}{3} g_{2}^{2} C_{H \square}+\left(-\frac{3}{2} g_{2}^{2}+6 g_{1}^{2} \mathrm{y}_{H}^{2}\right) C_{H D}\right]-\left[3\left(3 \mathrm{y}_{q}^{2}+3 \mathrm{y}_{d}^{2}-4 \mathrm{y}_{q} \mathrm{y}_{d}\right) g_{1}^{2}+\frac{27}{4} g_{2}^{2}+6 c_{F, 3} g_{3}^{2}\right] C_{r s} \\
& +3\left[Y_{d}^{\dagger}\right]_{r s}\left(8 c_{F, 3} g_{3}^{2} C_{H G}+3 g_{2}^{2} C_{H W}+4\left(\mathrm{y}_{H}^{2}+2 \mathrm{y}_{q} \mathrm{y}_{d}\right) g_{1}^{2} C_{H B}+2 \mathrm{y}_{q} g_{1} g_{2} C_{H W B}\right) \\
& -12\left[Y_{u}^{\dagger} Y_{u}\right]_{r t} g_{2} C_{d W}-6 g_{2} C_{u W}\left[Y_{u} Y_{d}^{\dagger}\right]_{t s}-3\left(4 c_{F, 3} g_{3} C_{d G}+g_{2} C_{d W}+\left(3 \mathrm{y}_{d}+\mathrm{y}_{u}\right) g_{1} C_{d B}\right)\left[Y_{d t} Y_{d}^{\dagger}\right]_{t s} \\
& -3\left[Y_{d}^{\dagger} Y_{d}\right]_{r t}\left(4 c_{F, 3} g_{3} C_{d s}-g_{2} C_{d s}+2\left(\mathrm{y}_{q}+\mathrm{y}_{d}\right) g_{1} C_{d s}\right)-6\left(4 g_{1 s}^{3} \mathrm{y}_{H}^{2} \mathrm{y}_{d}+4 g_{1}^{3} \mathrm{y}_{H}^{2} \mathrm{y}_{q}+g_{2}^{2} g_{1} \mathrm{y}_{H}\right) C_{d B} \\
& -3\left(4 g_{1}^{2} g_{2} \mathrm{y}_{H} \mathrm{y}_{d}+4 g_{1}^{2} g_{2} \mathrm{y}_{H} \mathrm{y}_{q}+3 g_{2}^{3}\right) C_{d S}+\left(3 g_{2}^{2}+12 g_{1}^{2} \mathrm{y}_{q} \mathrm{y}_{H}\right)\left[Y_{d}^{\dagger}\right]_{r t} C_{H d} \\
& +3 g_{2}^{2}\left[Y_{u}^{\dagger}\right]_{r t} C_{t s}^{H_{t s}}+12 g_{1}^{2} \mathrm{y}_{d} \mathrm{y}_{H} C_{r t}^{(1)}\left[Y_{d}^{\dagger}\right]_{t s}+12 g_{1}^{2} \mathrm{y}_{d} \mathrm{y}_{H} C_{r q}^{(3)}\left[Y_{d}^{\dagger}\right]_{t s}+\frac{4}{3} g_{2}^{2}\left[Y_{d}^{\dagger}\right]_{r s}\left(\underset{t t}{C_{t l}^{(3)}}+N_{c} C_{t q}^{(3)}\right)
\end{aligned}
$$




\section{C.6 $\psi^{2} X H$}

$$
\begin{aligned}
& \dot{C}_{r s}=\left[\left(3 c_{F, 2}-b_{0,2}\right) g_{2}^{2}+\left(-3 \mathrm{y}_{e}^{2}+8 \mathrm{y}_{e} \mathrm{y}_{l}-3 \mathrm{y}_{l}^{2}\right) g_{1}^{2}\right] C_{r s}+g_{1} g_{2}\left(3 \mathrm{y}_{l}-\mathrm{y}_{e}\right) C_{r s} \\
& -\left[Y_{e}^{\dagger}\right]_{r s}\left(g_{2}\left(C_{H W}+i C_{H \widetilde{W}}\right)+g_{1}\left(\mathrm{y}_{l}+\mathrm{y}_{e}\right)\left(C_{H W B}+i C_{H \widetilde{W} B}\right)\right) \\
& \underset{r s}{\dot{C}_{e B}}=\left[-3 c_{F, 2} g_{2}^{2}+\left(3 \mathrm{y}_{e}^{2}+4 \mathrm{y}_{e} \mathrm{y}_{l}+3 \mathrm{y}_{l}^{2}-b_{0,1}\right) g_{1}^{2}\right] C_{r s}+4 c_{F, 2} g_{1} g_{2}\left(3 \mathrm{y}_{l}-\mathrm{y}_{e}\right) C_{e W} \\
& -\left[Y_{e}^{\dagger}\right]_{r s}\left(2 g_{1}\left(\mathrm{y}_{l}+\mathrm{y}_{e}\right)\left(C_{H B}+i C_{H \widetilde{B}}\right)+\frac{3}{2} g_{2}\left(C_{H W B}+i C_{H \widetilde{W} B}\right)\right) \\
& \dot{C}_{r s}=\left[\left(10 c_{F, 3}-4 c_{A, 3}-b_{0,3}\right) g_{3}^{2}-3 c_{F, 2} g_{2}^{2}+\left(-3 \mathrm{y}_{u}^{2}+8 \mathrm{y}_{u} \mathrm{y}_{q}-3 \mathrm{y}_{q}^{2}\right) g_{1}^{2}\right] C_{u G} \\
& +8 c_{F, 2} g_{2} g_{3} C_{u W}+4 g_{1} g_{3}\left(\mathrm{y}_{u}+\mathrm{y}_{q}\right) C_{u_{s}}-4\left[Y_{u}^{\dagger}\right]_{r s} g_{3}\left(C_{H G}+i C_{H \widetilde{G}}\right)-3 g_{3}^{2} c_{A, 3}\left[Y_{u}^{\dagger}\right]_{r s}\left(C_{G}+i C_{\widetilde{G}}\right) \\
& \dot{C}_{u W}=\left[2 c_{F, 3} g_{3}^{2}+\left(3 c_{F, 2}-b_{0,2}\right) g_{2}^{2}+\left(-3 \mathrm{y}_{u}^{2}+8 \mathrm{y}_{u} \mathrm{y}_{q}-3 \mathrm{y}_{q}^{2}\right) g_{1}^{2}\right] C_{u W}+2 c_{F, 3} g_{2} g_{3} C_{u G} \\
& +g_{1} g_{2}\left(3 \mathrm{y}_{q}-\mathrm{y}_{u}\right) C_{u B}-\left[Y_{u}^{\dagger}\right]_{r s}\left(g_{2}\left(C_{H W}+i C_{H \widetilde{W}}\right)+g_{1}\left(\mathrm{y}_{q}+\mathrm{y}_{u}\right)\left(C_{H W B}+i C_{H \widetilde{W} B}\right)\right) \\
& \dot{C}_{r s}=\left[2 c_{F, 3} g_{3}^{2}-3 c_{F, 2} g_{2}^{2}+\left(3 \mathrm{y}_{u}^{2}+4 \mathrm{y}_{u} \mathrm{y}_{q}+3 \mathrm{y}_{q}^{2}-b_{0,1}\right) g_{1}^{2}\right] C_{r s}+4 c_{F, 3} g_{1} g_{3}\left(\mathrm{y}_{u}+\mathrm{y}_{q}\right) \underset{r s}{C_{u G}} \\
& +4 c_{F, 2} g_{1} g_{2}\left(3 \mathrm{y}_{q}-\mathrm{y}_{u}\right) C_{r W}-\left[Y_{u}^{\dagger}\right]_{r s}\left(2 g_{1}\left(\mathrm{y}_{q}+\mathrm{y}_{u}\right)\left(C_{H B}+i C_{H \widetilde{B}}\right)+\frac{3}{2} g_{2}\left(C_{H W B}+i C_{H \widetilde{W} B}\right)\right) \\
& \dot{C}_{d G}=\left[\left(10 c_{F, 3}-4 c_{A, 3}-b_{0,3}\right) g_{3}^{2}-3 c_{F, 2} g_{2}^{2}+\left(-3 \mathrm{y}_{d}^{2}+8 \mathrm{y}_{d} \mathrm{y}_{q}-3 \mathrm{y}_{q}^{2}\right) g_{1}^{2}\right] C_{r_{s}} \\
& +8 c_{F, 2} g_{2} g_{3} C_{d S}+4 g_{1} g_{3}\left(\mathrm{y}_{d}+\mathrm{y}_{q}\right) C_{d B}-4\left[Y_{d}^{\dagger}\right]_{r s} g_{3}\left(C_{H G}+i C_{H \widetilde{G}}\right)-3 g_{3}^{2} c_{A, 3}\left[Y_{d}^{\dagger}\right]_{r s}\left(C_{G}+i C_{\widetilde{G}}\right) \\
& \dot{C}_{d W}=\left[2 c_{F, 3} g_{3}^{2}+\left(3 c_{F, 2}-b_{0,2}\right) g_{2}^{2}+\left(-3 \mathrm{y}_{d}^{2}+8 \mathrm{y}_{d} \mathrm{y}_{q}-3 \mathrm{y}_{q}^{2}\right) g_{1}^{2}\right] C_{d W}+2 c_{F, 3} g_{2} g_{3} C_{d G} \\
& +g_{1} g_{2}\left(3 \mathrm{y}_{q}-\mathrm{y}_{d}\right) C_{d B}-\left[Y_{d}^{\dagger}\right]_{r s}\left(g_{2}\left(C_{H W}+i C_{H \widetilde{W}}\right)+g_{1}\left(\mathrm{y}_{q}+\mathrm{y}_{d}\right)\left(C_{H W B}+i C_{H \widetilde{W} B}\right)\right) \\
& \underset{C_{d B}}{\dot{C}_{d B}}=\left[2 c_{F, 3} g_{3}^{2}-3 c_{F, 2} g_{2}^{2}+\left(3 \mathrm{y}_{d}^{2}+4 \mathrm{y}_{d} \mathrm{y}_{q}+3 \mathrm{y}_{q}^{2}-b_{0,1}\right) g_{1}^{2}\right] C_{r s}+4 c_{F, 3} g_{1} g_{3}\left(\mathrm{y}_{d}+\mathrm{y}_{q}\right) C_{d G} \\
& +4 c_{F, 2} g_{1} g_{2}\left(3 \mathrm{y}_{q}-\mathrm{y}_{d}\right) C_{d W}-\left[Y_{d}^{\dagger}\right]_{r s}\left(2 g_{1}\left(\mathrm{y}_{q}+\mathrm{y}_{d}\right)\left(C_{H B}+i C_{H \widetilde{B}}\right)+\frac{3}{2} g_{2}\left(C_{H W B}+i C_{H \widetilde{W} B}\right)\right)
\end{aligned}
$$

\section{C. $7 \psi^{2} H^{2} D$}

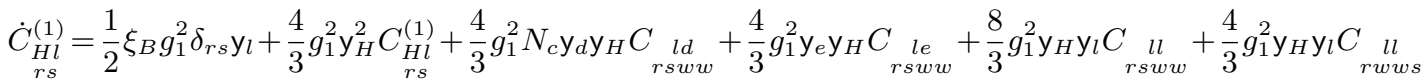

$$
\begin{aligned}
& +\frac{4}{3} g_{1}^{2} \mathrm{y}_{H} \mathrm{y}_{l} C_{w s r w}^{l l}+\frac{8}{3} g_{1}^{2} \mathrm{y}_{H} \mathrm{y}_{l} C_{w w r s}^{l l}+\frac{8}{3} g_{1}^{2} N_{c} \mathrm{y}_{H} \mathrm{y}_{q} C_{\substack{l q \\
r s w w}}^{(1)}+\frac{4}{3} g_{1}^{2} N_{c} \mathrm{y}_{H} \mathrm{y}_{u} C_{r s w w}^{l u} \\
& \dot{C}_{r l}^{(3)}=\frac{1}{6} g_{2}^{2} C_{H \square} \delta_{r s}+\frac{2}{3} g_{2}^{2} C_{t t}^{(3)} \delta_{r s}+\frac{2}{3} g_{2}^{2} N_{c} C_{H q}^{(3)} \delta_{r s}+\frac{1}{3} g_{2}^{2} C_{r l}^{(3)}+\frac{1}{3} g_{2}^{2} C_{r w w s}^{l l}+\frac{1}{3} g_{2}^{2} C_{w s r w}^{l l}+\frac{2}{3} g_{2}^{2} N_{c} C_{r s w w}^{(3)} \\
& -6 g_{2}^{2} C_{r s}^{(3)} \\
& \dot{C}_{r e}=\frac{1}{2} \xi_{B} g_{1}^{2} \delta_{r s} \mathrm{y}_{e}+\frac{4}{3} g_{1}^{2} \mathrm{y}_{H}^{2} C_{r s}^{H e}+\frac{4}{3} g_{1}^{2} N_{c} \mathrm{y}_{d} \mathrm{y}_{H} C_{r s w w}^{e d}+\frac{4}{3} g_{1}^{2} \mathrm{y}_{e} \mathrm{y}_{H} C_{r s w w}^{e e}+\frac{4}{3} g_{1}^{2} \mathrm{y}_{e} \mathrm{y}_{H} C_{r w w s}^{e e}+\frac{4}{3} g_{1}^{2} \mathrm{y}_{e} \mathrm{y}_{H} C_{w s r w}^{e e} \\
& +\frac{4}{3} g_{1}^{2} \mathrm{y}_{e} \mathrm{y}_{H} C_{w w r s}^{e e}+\frac{4}{3} g_{1}^{2} N_{c} \mathrm{y}_{H} \mathrm{y}_{u} C_{r s w w}^{e e}+\frac{8}{3} g_{1}^{2} \mathrm{y}_{H} \mathrm{y}_{l} C_{w w r s}^{l e}+\frac{8}{3} g_{1}^{2} N_{c} \mathrm{y}_{H} \mathrm{y}_{q} C_{w w r s}^{q e} \\
& \dot{C}_{r q}^{(1)}=\frac{1}{2} \xi_{B} g_{1}^{2} \delta_{r s} \mathrm{y}_{q}+\frac{4}{3} g_{1}^{2} \mathrm{y}_{H}^{2} C_{r s}^{(1)}+\frac{8}{3} g_{1}^{2} \mathrm{y}_{H} \mathrm{y}_{l} C_{\substack{l q \\
w w r s}}^{(1)}+\frac{4}{3} g_{1}^{2} N_{c} \mathrm{y}_{d} \mathrm{y}_{H} C_{r s w w}^{(1)}+\frac{4}{3} g_{1}^{2} \mathrm{y}_{e} \mathrm{y}_{H} C_{r s w w}^{q e}+\frac{8}{3} g_{1}^{2} N_{c} \mathrm{y}_{H} \mathrm{y}_{q} C_{r s w w}^{(1)}
\end{aligned}
$$

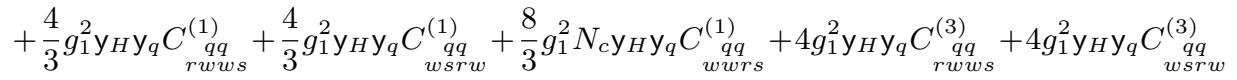

$$
\begin{aligned}
& +\frac{4}{3} g_{1}^{2} N_{c} \mathrm{y}_{H} \mathrm{y}_{u} C_{\substack{q u \\
r s w}}^{(1)}
\end{aligned}
$$

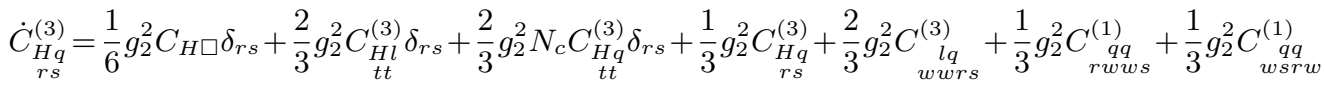

$$
\begin{aligned}
& +\frac{2}{3} g_{2}^{2} N_{c} C_{r s w w}^{(3)}-\frac{1}{3} g_{2}^{2} C_{r w w s}^{(3)}-\frac{1}{3 q} g_{2}^{2} C_{w s r w}^{(3)}+\frac{2}{3 q} g_{2}^{2} N_{c} C_{\substack{q q \\
\text { rwrs }}}^{(3)}
\end{aligned}
$$




$$
\begin{aligned}
& \dot{C}_{r s}=\frac{1}{2} \xi_{B} g_{1}^{2} \delta_{r s} \mathrm{y}_{u}+\frac{4}{3} g_{1}^{2} \mathrm{y}_{H}^{2} C_{r s}+\frac{4}{3} g_{1}^{2} \mathrm{y}_{e} \mathrm{y}_{H} C_{w w r s}^{e u}+\frac{8}{3} g_{1}^{2} \mathrm{y}_{H} \mathrm{y}_{l} C_{w w r s} \underset{w}{w}+\frac{8}{3} g_{1}^{2} N_{c} \mathrm{y}_{H} \mathrm{y}_{q} C_{\underset{w w r s}{(1)}}^{(1)}+\frac{4}{3} g_{1}^{2} N_{c} \mathrm{y}_{d} \mathrm{y}_{H} C_{\substack{u d \\
\text { wsw }}}^{(1)} \\
& +\frac{4}{3} g_{1}^{2} N_{c} \mathrm{y}_{H} \mathrm{y}_{u} C_{r s w w}^{u u}+\frac{4}{3} g_{1}^{2} \mathrm{y}_{H} \mathrm{y}_{u} C_{r w w s}^{u u}+\frac{4}{3} g_{1}^{2} \mathrm{y}_{H} \mathrm{y}_{u} C_{\text {wsrw }}^{u u}+\frac{4}{3} g_{1}^{2} N_{c} \mathrm{y}_{H} \mathrm{y}_{u} C_{w w}^{u u} \\
& \dot{C}_{r d}=\frac{1}{2} \xi_{B} g_{1}^{2} \delta_{r s} \mathrm{y}_{d}+\frac{4}{3} g_{1}^{2} y_{H}^{2} C_{r s}+\frac{4}{3} g_{1}^{2} N_{c} \mathrm{y}_{d} \mathrm{y}_{H} C_{r s w w}^{d d}+\frac{4}{3} g_{1}^{2} \mathrm{y}_{d} \mathrm{y}_{H} C_{r w w s}^{d d}+\frac{4}{3} g_{1}^{2} \mathrm{y}_{d} \mathrm{y}_{H} C_{w s r w}^{d d}+\frac{4}{3} g_{1}^{2} N_{c} \mathrm{y}_{d} \mathrm{y}_{H} C_{w w r s}^{d d} \\
& +\frac{4}{3} g_{1}^{2} \mathrm{y}_{e} \mathrm{y}_{H} C_{\text {ed }}^{e d s}+\frac{8}{3} g_{1}^{2} \mathrm{y}_{H} \mathrm{y}_{l} C_{w w r s}^{l d}+\frac{8}{3} g_{1}^{2} N_{c} \mathrm{y}_{H} \mathrm{y}_{q} C_{\substack{q d \\
\text { wwrs }}}^{(1)}+\frac{4}{3} g_{1}^{2} N_{c} \mathrm{y}_{H} \mathrm{y}_{u} C_{\substack{u d r \\
w w}}^{(1)} \\
& \dot{C}_{r s}=-3 g_{1}^{2}\left(\mathrm{y}_{u}-\mathrm{y}_{d}\right)^{2} C_{H u d}
\end{aligned}
$$

\section{C. $8 \psi^{4}$}

\section{C.8.1 $(\bar{L} L)(\bar{L} L)$}

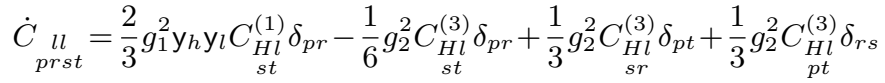

$$
\begin{aligned}
& +\frac{2}{3} g_{1}^{2} \mathrm{y}_{h} \mathrm{y}_{l} C_{H r}^{(1)} \delta_{s t}-\frac{1}{6} g_{2}^{2} C_{H r}^{(3)} \delta_{s t}+\frac{4}{3} g_{1}^{2} \mathrm{y}_{l}^{2} C_{p r w w}^{l l} \delta_{s t}+\frac{4}{3} g_{1}^{2} y_{l}^{2} C_{s t w w}^{l l} \delta_{p r}+\frac{4}{3} g_{1}^{2} \mathrm{y}_{l}^{2} C_{w w s t}^{l l} \delta_{p r} \\
& +\frac{4}{3} g_{1}^{2} \mathrm{y}_{l}^{2} C_{\text {wwpr }}^{l l} \delta_{s t}+\frac{2}{3} g_{1}^{2} \mathrm{y}_{l}^{2} C \underset{p w w r}{l l} \delta_{s t}+\frac{2}{3} g_{1}^{2} \mathrm{y}_{l}^{2} C \underset{\text { swwt }}{l l} \delta_{p r}+\frac{2}{3} g_{1}^{2} \mathrm{y}_{l}^{2} C_{\text {wrpw }}^{l l} \delta_{s t}+\frac{2}{3} g_{1}^{2} \mathrm{y}_{l}^{2} C \underset{w t s w}{l l} \delta_{p r} \\
& -\frac{1}{6} g_{2}^{2} C \underset{p w w r}{l l} \delta_{s t}-\frac{1}{6} g_{2}^{2} C \underset{\text { swwt }}{u l} \delta_{p r}-\frac{1}{6} g_{2}^{2} C \underset{w r p w}{l l} \delta_{s t}-\frac{1}{6} g_{2}^{2} C_{w t s w}^{u l} \delta_{p r}+\frac{1}{3} g_{2}^{2} C_{s w w r}^{l l} \delta_{p t} \\
& +\frac{1}{3} g_{2}^{2} C_{p w w t}^{l l} \delta_{r s}+\frac{1}{3} g_{2}^{2} C_{w r s w}^{l l} \delta_{p t}+\frac{1}{3} g_{2}^{2} C_{w t p w}^{l l} \delta_{r s}+\frac{4}{3} g_{1}^{2} N_{c} y_{l y} C_{q} C_{p q w w}^{(1)} \delta_{s t}+\frac{4}{3} g_{1}^{2} N_{c} y_{l} \mathrm{y}_{q} C_{\substack{l q \\
\text { stww }}}^{(1)} \delta_{p r}
\end{aligned}
$$

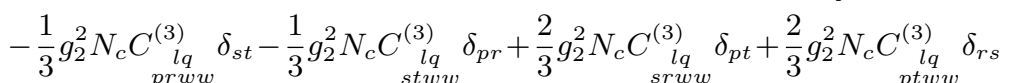

$$
\begin{aligned}
& +\frac{2}{3} g_{1}^{2} N_{c} \mathrm{y}_{l} \mathrm{y}_{u} C_{\text {prww }}^{\text {lu }} \delta_{s t}+\frac{2}{3} g_{1}^{2} N_{c} \mathrm{y}_{l} \mathrm{y}_{u} C_{\text {stww }}^{l u} \delta_{p r}+\frac{2}{3} g_{1}^{2} N_{c} \mathrm{y}_{d} \mathrm{y}_{l} C_{\text {prww }}^{l d} \delta_{s t}+\frac{2}{3} g_{1}^{2} N_{c} \mathrm{y}_{d} \mathrm{y}_{l} C_{\text {stww }}^{l d} \delta_{p r} \\
& +\frac{2}{3} g_{1}^{2} \mathrm{y}_{e} \mathrm{y}_{l} C_{p r w w}^{l e} \delta_{s t}+\frac{2}{3} g_{1}^{2} \mathrm{y}_{e} \mathrm{y}_{l} C_{\text {stww }}^{l e} \delta_{p r}+6 g_{2}^{2} C_{p t s r}^{l l}-3\left(g_{2}^{2}-4 y_{l}^{2} g_{1}^{2}\right) C_{p r s t}^{l l} \\
& \dot{C}_{p r s t}^{(1)}=\frac{2}{3} g_{1}^{2} \mathrm{y}_{h} \mathrm{y}_{q} C_{H q}^{(1)} \delta_{p r}+\frac{2}{3} g_{1}^{2} \mathrm{y}_{h} \mathrm{y}_{q} C_{p r}^{(1)} \delta_{s t}+\frac{4}{3} g_{1}^{2} \mathrm{y}_{l} \mathrm{y}_{q} C_{\substack{l q \\
\text { wwst }}}^{(1)} \delta_{p r} \\
& +\frac{4}{3} g_{1}^{2} \mathrm{y}_{l} \mathrm{y}_{q} C_{\substack{l q \\
\text { wwpr }}}^{(1)} \delta_{s t}+\frac{4}{3} g_{1}^{2} N_{c} y_{q}^{2} C_{\substack{q q \\
p r w}}^{(1)} \delta_{s t}+\frac{4}{3} g_{1}^{2} N_{c} \mathrm{y}_{q}^{2} C_{\substack{q q \\
\text { stww }}}^{(1)} \delta_{p r}+\frac{4}{3} g_{1}^{2} N_{c} \mathrm{y}_{q}^{2} C_{\substack{q u \\
w w t}}^{(1)} \delta_{p r}+\frac{4}{3} g_{1}^{2} N_{c} y_{q}^{2} C_{\underset{w w p r}{(1)}}^{(1)} \delta_{s t}
\end{aligned}
$$

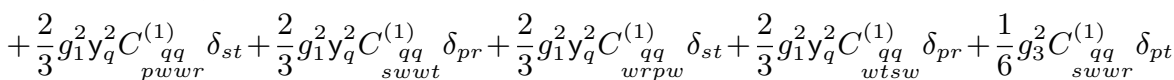

$$
\begin{aligned}
& +\frac{1}{6} g_{3}^{2} C_{\substack{q q \\
p w w t}}^{(1)} \delta_{r s}+\frac{1}{6} g_{3}^{2} C_{\substack{q q \\
w r w w}}^{(1)} \delta_{p t}+\frac{1}{6} g_{3}^{2} C_{\substack{q q \\
w t p w}}^{(1)} \delta_{r s}-\frac{1}{3 N_{c}} g_{3}^{2} C_{\substack{q q \\
\text { www }}}^{(1)} \delta_{s t}-\frac{1}{3 N_{c}} g_{3}^{2} C_{\substack{q q \\
\text { swwt }}}^{(1)} \delta_{p r}
\end{aligned}
$$

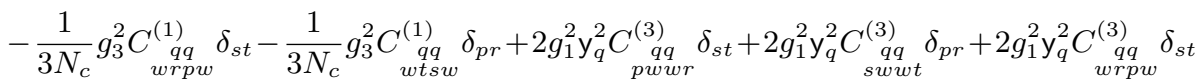

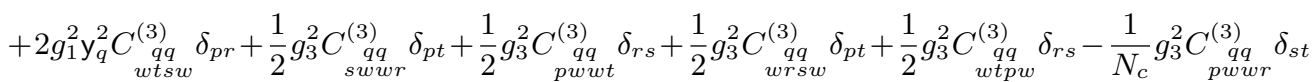

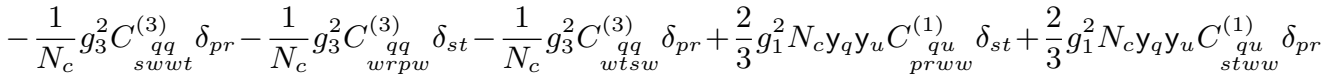

$$
\begin{aligned}
& +\frac{2}{3} g_{1}^{2} N_{c} \mathrm{y}_{d} \mathrm{y}_{q} C_{\substack{q d \\
p r w w}}^{(1)} \delta_{s t}+\frac{2}{3} g_{1}^{2} N_{c} \mathrm{y}_{d} \mathrm{y}_{q} C_{\substack{q d \\
s t w w}}^{(1)} \delta_{p r}+\frac{1}{12} g_{3}^{2} C_{\substack{q u \\
s r w w}}^{(8)} \delta_{p t}+\frac{1}{12} g_{3}^{2} C_{\substack{q u \\
p t w w}}^{(8)} \delta_{r s}-\frac{1}{6 N_{c}} g_{3}^{2} C_{\substack{q u \\
p r w w}}^{(8)} \delta_{s t} \\
& -\frac{1}{6 N_{c}} g_{3}^{2} C_{\substack{q u \\
s t w w}}^{(8)} \delta_{p r}+\frac{1}{12} g_{3}^{2} C_{\substack{q d \\
s r w w}}^{(8)} \delta_{p t}+\frac{1}{12} g_{3}^{2} C_{\substack{q d \\
p t w w}}^{(8)} \delta_{r s}-\frac{1}{6 N_{c}} g_{3}^{2} C_{\substack{q d \\
p r w w}}^{(8)} \delta_{s t}-\frac{1}{6 N_{c}} g_{3}^{2} C_{\substack{q d \\
\text { stww }}}^{(8)} \delta_{p r}
\end{aligned}
$$

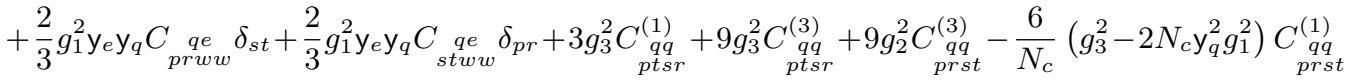




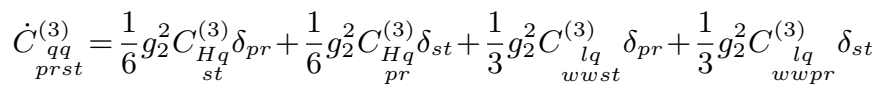

$$
\begin{aligned}
& +\frac{1}{6} g_{2}^{2} C_{\substack{q q \\
\text { swt }}}^{(1)} \delta_{p r}+\frac{1}{6} g_{2}^{2} C_{w t s w}^{(1)} \delta_{p r}+\frac{1}{6} g_{2}^{2} C_{p w w r}^{(1)} \delta_{p t}+\frac{1}{6} g_{2}^{2} C_{w r p w}^{(1)} \delta_{w t}^{q q}+\frac{1}{6} g_{3}^{2} C_{p w w t}^{(1)} \delta_{r s}
\end{aligned}
$$

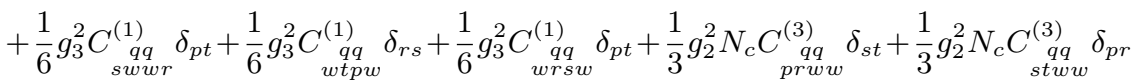

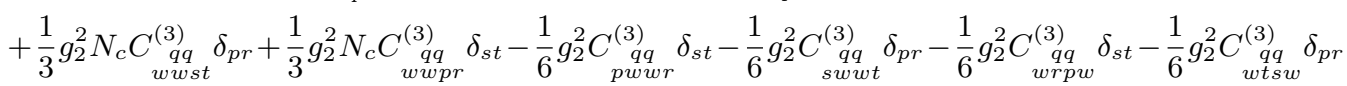

$$
\begin{aligned}
& +\frac{1}{2} g_{3}^{2} C_{\substack{q q \\
p w t}}^{(3)} \delta_{r s}+\frac{1}{2} g_{3}^{2} C_{\substack{q q \\
\text { swr }}}^{(3)} \delta_{p t}+\frac{1}{2} g_{3}^{2} C_{\substack{q q \\
w t p w}}^{(3)} \delta_{r s}+\frac{1}{2} g_{3}^{2} C_{w r s w}^{(3)} \delta_{p t} \\
& +\frac{1}{12} g_{3}^{2} C_{p t w w}^{(8)} \delta_{r s}+\frac{1}{12} g_{3}^{2} C_{s r w w}^{(8)} \delta_{p t}^{q u}+\frac{1}{12} g_{3}^{2} C_{\substack{q d \\
\text { ptww }}}^{(8)} \delta_{r s}+\frac{1}{12} g_{3}^{2} C_{\substack{q d \\
\text { stww }}}^{(8)} \delta_{p t} \\
& -3 g_{3}^{2} C_{p t s r}^{(3)}-\frac{6}{N_{c}} g_{3}^{2} C_{p r s t}^{(3)}-6 g_{2}^{2} C_{p r s t}^{(3)}+12 y_{q}^{2} g_{1}^{2} C_{p r s t}^{(3)}+3 g_{3}^{2} C_{p t s r}^{(1)}+3 g_{2}^{2} C_{p r s t}^{(1)} \\
& \underset{p r s t}{\dot{C}_{l q}^{(1)}}=\frac{4}{3} g_{1}^{2} \mathrm{y}_{h} \mathrm{y}_{l} C_{\substack{H q \\
s t}}^{(1)} \delta_{p r}+\frac{4}{3} g_{1}^{2} \mathrm{y}_{h} \mathrm{y}_{q} C_{p r}^{(1)} \delta_{s t}+\frac{8}{3} g_{1}^{2} \mathrm{y}_{l} \mathrm{y}_{q} C_{p r w w}^{l l} \delta_{s t}+\frac{8}{3} g_{1}^{2} \mathrm{y}_{l} \mathrm{y}_{q} C_{w w p r}^{l l} \delta_{s t}+\frac{4}{3} g_{1}^{2} \mathrm{y}_{l} \mathrm{y}_{q} C_{p w w r} l l \delta_{s t} \\
& +\frac{4}{3} g_{1}^{2} \mathrm{y}_{l} \mathrm{y}_{q} C_{w r p w}^{l l} \delta_{s t}+\frac{8}{3} g_{1}^{2} N_{c} \mathrm{y}_{q}^{2} C_{p r w w}^{(1)} \delta_{s t}+\frac{8}{3} g_{1}^{2} \mathrm{y}_{l}^{2} C_{\substack{l q \\
w w s t}}^{(1)} \delta_{p r}+\frac{8}{3} g_{1}^{2} N_{c} \mathrm{y}_{l} \mathrm{y}_{q} C_{\substack{q q \\
\text { stww }}}^{(1)} \delta_{p r}+\frac{8}{3} g_{1}^{2} N_{c} \mathrm{y}_{l} \mathrm{y}_{q} C_{\underset{w w s t}{(1)}}^{(1)} \delta_{p r} \\
& +\frac{4}{3} g_{1}^{2} \mathrm{y}_{l} \mathrm{y}_{q} C_{\substack{q q \\
s w w}}^{(1)} \delta_{p r}+\frac{4}{3} g_{1}^{2} \mathrm{y}_{l} \mathrm{y}_{q} C_{\substack{q q \\
w t s w}}^{(1)} \delta_{p r}+4 g_{1}^{2} \mathrm{y}_{l} \mathrm{y}_{q} C_{\substack{q q \\
s w t}}^{(3)} \delta_{p r}+4 g_{1}^{2} \mathrm{y}_{l} \mathrm{y}_{q} C_{\substack{q q \\
w t s w}}^{(3)} \delta_{p r}+\frac{4}{3} g_{1}^{2} N_{c} \mathrm{y}_{l} \mathrm{y}_{u} C_{\substack{q u \\
s t w w}}^{(1)} \delta_{p r} \\
& +\frac{4}{3} g_{1}^{2} N_{c} \mathrm{y}_{d} \mathrm{y}_{l} C_{\substack{q d \\
\text { stww }}}^{(1)} \delta_{p r}+\frac{4}{3} g_{1}^{2} \mathrm{y}_{e} \mathrm{y}_{l} C_{\text {stww }}^{q e} \delta_{p r}+\frac{4}{3} g_{1}^{2} N_{c} \mathrm{y}_{q} \mathrm{y}_{u} C_{\text {prww }}^{l u} \delta_{s t}+\frac{4}{3} g_{1}^{2} N_{c} \mathrm{y}_{d} \mathrm{y}_{q} C_{\text {prww }}^{l d} \delta_{s t} \\
& +\frac{4}{3} g_{1}^{2} \mathrm{y}_{e} \mathrm{y}_{q} C_{\text {prww }}^{l e} \delta_{s t}+12 \mathrm{y}_{l} \mathrm{y}_{q} g_{1}^{2} C_{\text {prst }}^{(1)}+9 g_{2}^{2} C_{\text {prst }}^{(3)}
\end{aligned}
$$

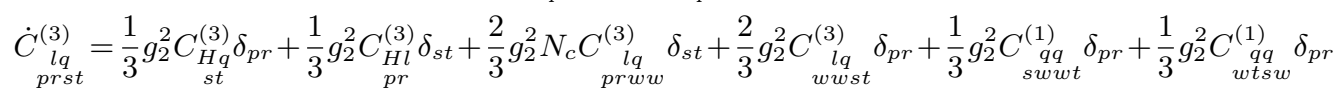

$$
\begin{aligned}
& +\frac{2}{3} g_{2}^{2} N_{c} C_{\substack{q q \\
\text { stww }}}^{(3)} \delta_{p r}+\frac{2}{3} g_{2}^{2} N_{c} C_{\text {wwst }}^{(3)} \delta_{p r}-\frac{1}{3} g_{2}^{2} C_{\substack{q q \\
\text { swt }}}^{(3)} \delta_{p r}-\frac{1}{3} g_{2}^{2} C_{\substack{q q \\
\text { wtsw }}}^{(3)} \delta_{p r} \\
& +\frac{1}{3} g_{2}^{2} C_{p w w r}^{l l} \delta_{s t}+\frac{1}{3} g_{2}^{2} C_{w r p w}^{l l} \delta_{s t}+3 g_{2}^{2} C_{\text {prst }}^{(1)}-6\left(g_{2}^{2}-2 \mathrm{y}_{l} \mathrm{y}_{q} g_{1}^{2}\right) C_{\text {prst }}^{(3)}
\end{aligned}
$$

\section{C.8.2 $(\bar{R} R)(\bar{R} R)$}

$\dot{C}_{p r s t}^{e e}=\frac{2}{3} g_{1}^{2} \mathrm{y}_{e} \mathrm{y}_{h} C_{H_{e}} \delta_{p r}+\frac{2}{3} g_{1}^{2} \mathrm{y}_{e} \mathrm{y}_{h} C_{p r}^{H e} \delta_{s t}+\frac{4}{3} g_{1}^{2} \mathrm{y}_{e} \mathrm{y}_{l} C_{w w p r}^{l e} \delta_{s t}+\frac{4}{3} g_{1}^{2} \mathrm{y}_{e} \mathrm{y}_{l} C_{w w s t}^{l e} \delta_{p r}+\frac{4}{3} g_{1}^{2} N_{c} \mathrm{y}_{e} \mathrm{y}_{q} C_{w w p r}^{q e} \delta_{s t}$

$+\frac{4}{3} g_{1}^{2} N_{c} \mathrm{y}_{e} \mathrm{y}_{q} C_{\text {wwst }}^{q e} \delta_{p r}+\frac{2}{3} g_{1}^{2} N_{c} \mathrm{y}_{e} \mathrm{y}_{u} C_{p r w w}^{\text {eu }} \delta_{s t}+\frac{2}{3} g_{1}^{2} N_{c} \mathrm{y}_{e} \mathrm{y}_{u} C_{\text {stww }}^{e u} \delta_{p r}+\frac{2}{3} g_{1}^{2} N_{c} \mathrm{y}_{d} \mathrm{y}_{e} C_{\text {prww }}^{e d} \delta_{s t}$

$+\frac{2}{3} g_{1}^{2} N_{c} \mathrm{y}_{d} \mathrm{y}_{e} C_{\text {stww }}^{e d} \delta_{p r}+\frac{2}{3} g_{1}^{2} \mathrm{y}_{e}^{2} C_{\text {prww }}^{e e} \delta_{s t}+\frac{2}{3} g_{1}^{2} \mathrm{y}_{e}^{2} C_{\text {stww }}^{e e} \delta_{p r}+\frac{2}{3} g_{1}^{2} \mathrm{y}_{e}^{2} C_{w w p r}^{e e} \delta_{s t}+\frac{2}{3} g_{1}^{2} \mathrm{y}_{e}^{2} C_{\text {wwst }}^{e e} \delta_{p r}$

$+\frac{2}{3} g_{1}^{2} \mathrm{y}_{e}^{2} C_{\text {pee }}^{e e} \delta_{s t}+\frac{2}{3} g_{1}^{2} \mathrm{y}_{e}^{2} C_{\text {swwt }}^{e e} \delta_{p r}+\frac{2}{3} g_{1}^{2} \mathrm{y}_{e}^{2} C_{w t s w}^{e e} \delta_{p r}+\frac{2}{3} g_{1}^{2} \mathrm{y}_{e}^{2} C_{w r p w}^{e e} \delta_{s t}+12 \mathrm{y}_{e}^{2} g_{1}^{2} C_{\text {prst }}^{e e}$

$\underset{\text { prst }}{\dot{C}_{u u}}=\frac{2}{3} g_{1}^{2} \mathrm{y}_{h} \mathrm{y}_{u} C_{\text {Hu }} \delta_{p r}+\frac{2}{3} g_{1}^{2} \mathrm{y}_{h} \mathrm{y}_{u} C_{p r}{ }_{p r} \delta_{s t}$

$+\frac{2}{3} g_{1}^{2} \mathrm{y}_{e} \mathrm{y}_{u} C_{w w s t}^{e u} \delta_{p r}+\frac{2}{3} g_{1}^{2} \mathrm{y}_{e} \mathrm{y}_{u} C_{w w p r}^{e u} \delta_{s t}+\frac{4}{3} g_{1}^{2} \mathrm{y}_{l} \mathrm{y}_{u} C_{w w p r}^{l u} \delta_{s t}+\frac{4}{3} g_{1}^{2} \mathrm{y}_{l} \mathrm{y}_{u} C_{w w s t}^{l u} \delta_{p r}$

$+\frac{4}{3} g_{1}^{2} N_{c} \mathrm{y}_{q} \mathrm{y}_{u} C_{\substack{q u \\ w w t}}^{(1)} \delta_{p r}+\frac{4}{3} g_{1}^{2} N_{c} \mathrm{y}_{q} \mathrm{y}_{u} C_{\underset{w w p r}{(1)}}^{(1)} \delta_{s t}+\frac{1}{3} g_{3}^{2} C_{w w p t}^{(8)} \delta_{r s}+\frac{1}{3} g_{3}^{2} C_{w w s r}^{(8)} \delta_{p t}-\frac{1}{3 N_{c}} g_{3}^{2} C_{w w u}^{(8)} \delta_{w r}$

$-\frac{1}{3 N_{c}} g_{3}^{2} C_{\substack{q u \\ w w p}}^{(8)} \delta_{s t}+\frac{2}{3} g_{1}^{2} N_{c} \mathrm{y}_{u}^{2} C_{p r w w}^{u u} \delta_{s t}+\frac{2}{3} g_{1}^{2} N_{c} \mathrm{y}_{u}^{2} C_{s t w w}^{u u} \delta_{p r}+\frac{2}{3} g_{1}^{2} N_{c} \mathrm{y}_{u}^{2} C_{w w p r}^{u u} \delta_{s t}+\frac{2}{3} g_{1}^{2} N_{c} \mathrm{y}_{u}^{2} C_{w w s t}^{u u} \delta_{p r}$

$+\frac{2}{3} g_{1}^{2} \mathrm{y}_{u}^{2} C_{p w w r}^{u u} \delta_{s t}+\frac{2}{3} g_{1}^{2} \mathrm{y}_{u}^{2} C_{\text {sww }}^{u u} \delta_{p r}+\frac{2}{3} g_{1}^{2} \mathrm{y}_{u}^{2} C_{w r p w}^{u u} \delta_{s t}+\frac{2}{3} g_{1}^{2} \mathrm{y}_{u}^{2} C_{w t s w}^{u u} \delta_{p r}+\frac{1}{3} g_{3}^{2} C_{p w w t}^{u u} \delta_{r s}$

$+\frac{1}{3} g_{3}^{2} C_{\text {sww } u \text { uu }} \delta_{p t}+\frac{1}{3} g_{3}^{2} C_{\text {wtpw }}^{u u} \delta_{r s}+\frac{1}{3} g_{3}^{2} C_{\text {wrsw }}^{\text {uu }} \delta_{p t}-\frac{1}{3 N_{c}} g_{3}^{2} C_{\text {pww }}^{u u} \delta_{s t}-\frac{1}{3 N_{c}} g_{3}^{2} C_{\text {swwt }}^{u u} \delta_{p r}$ 
$-\frac{1}{3 N_{c}} g_{3}^{2} C_{w r p w}^{u u} \delta_{s t}-\frac{1}{3 N_{c}} g_{3}^{2} C_{w u s w}^{u u} \delta_{p r}+\frac{2}{3} g_{1}^{2} N_{c} \mathrm{y}_{d} \mathrm{y}_{u} C_{\begin{array}{c}u d \\ \text { prww }\end{array}}^{(1)} \delta_{s t}+\frac{2}{3} g_{1}^{2} N_{c} \mathrm{y}_{d} \mathrm{y}_{u} C_{\substack{u d \\ \text { stww }}}^{(1)} \delta_{p r}+\frac{1}{6} g_{3}^{2} C_{\substack{u d \\ p t w w}}^{(8)} \delta_{r s}$

$+\frac{1}{6} g_{3}^{2} C_{\substack{u d \\ \text { srww }}}^{(8)} \delta_{p t}-\frac{1}{6 N_{c}} g_{3}^{2} C_{\substack{u d \\ \text { prww }}}^{(8)} \delta_{s t}-\frac{1}{6 N_{c}} g_{3}^{2} C_{\substack{u d \\ \text { stww }}}^{(8)} \delta_{p r}+6 g_{3}^{2} C_{p u t}^{u u}-6\left(\frac{1}{N_{c}} g_{3}^{2}-2 \mathrm{y}_{u}^{2} g_{1}^{2}\right) C_{\substack{u u \\ p r s t}}$

$\dot{C}_{\text {prst }}=\frac{2}{3} g_{1}^{2} \mathrm{y}_{d} \mathrm{y}_{h} C_{\text {st }} \delta_{p r}+\frac{2}{3} g_{1}^{2} \mathrm{y}_{d} \mathrm{y}_{h} C_{\text {pr }} \delta_{s t}$

$+\frac{2}{3} g_{1}^{2} N_{c} y_{d}^{2} C_{p r w w}^{d d} \delta_{s t}+\frac{2}{3} g_{1}^{2} N_{c} y_{d}^{2} C_{\text {stw }}^{d d} \delta_{p r}+\frac{2}{3} g_{1}^{2} N_{c} y_{d}^{2} C_{w w p r}^{d d} \delta_{s t}+\frac{2}{3} g_{1}^{2} N_{c} y_{d}^{2} C_{\text {wwst }}^{d d} \delta_{p r}$

$+\frac{2}{3} g_{1}^{2} y_{d}^{2} C_{p w w r}^{d d} \delta_{s t}+\frac{2}{3} g_{1}^{2} y_{d}^{2} C_{s w w t}^{d d} \delta_{p r}+\frac{2}{3} g_{1}^{2} y_{d}^{2} C_{w t s w}^{d d} \delta_{p r}+\frac{2}{3} g_{1}^{2} y_{d}^{2} C_{w r p w}^{d d} \delta_{s t}+\frac{1}{3} g_{3}^{2} C_{p w w t}^{d d} \delta_{r s}$

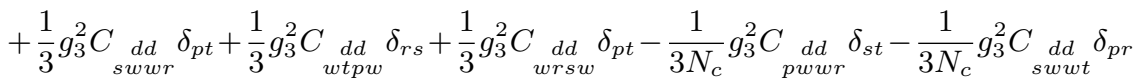

$-\frac{1}{3 N_{c}} g_{3}^{2} C_{w t s w}^{d d} \delta_{p r}-\frac{1}{3 N_{c}} g_{3}^{2} C_{w r p w}^{d d} \delta_{s t}+\frac{4}{3} g_{1}^{2} \mathrm{y}_{d} \mathrm{y}_{l} C_{w w p r}^{l d} \delta_{s t}+\frac{4}{3} g_{1}^{2} \mathrm{y}_{d} \mathrm{y}_{l} C_{w w s t}^{l d} \delta_{p r}+\frac{4}{3} g_{1}^{2} N_{c} \mathrm{y}_{d} \mathrm{y}_{q} C_{\substack{q d \\ w w p r}}^{(1)} \delta_{s t}$

$+\frac{4}{3} g_{1}^{2} N_{c} \mathrm{y}_{d} \mathrm{y}_{q} C_{\substack{q d \\ w w s t}}^{(1)} \delta_{p r}+\frac{1}{3} g_{3}^{2} C_{\underset{q d}{(8)}}^{(8)} \delta_{p t}+\frac{1}{3} g_{3}^{2} C_{\substack{q d \\ w w p t}}^{(8)} \delta_{r s}-\frac{1}{3 N_{c}} g_{3}^{2} C_{\underset{q d}{(8)}}^{(8)} \delta_{s t}-\frac{1}{3 N_{c}} g_{3}^{2} C_{\substack{q d \\ w w s t}}^{(8)} \delta_{p r}$

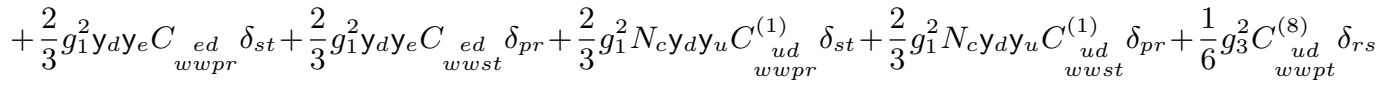

$+\frac{1}{6} g_{3}^{2} C_{\substack{u d \\ \text { wws }}}^{(8)} \delta_{p t}-\frac{1}{6 N_{c}} g_{3}^{2} C_{\substack{u d \\ w w r}}^{(8)} \delta_{s t}-\frac{1}{6 N_{c}} g_{3}^{2} C_{\substack{u d \\ \text { wwst }}}^{(8)} \delta_{p r}+6 g_{3}^{2} C_{\substack{d d \\ p t s r}}-6\left(\frac{1}{N_{c}} g_{3}^{2}-2 \mathrm{y}_{d}^{2} g_{1}^{2}\right) C_{\text {prst }}$

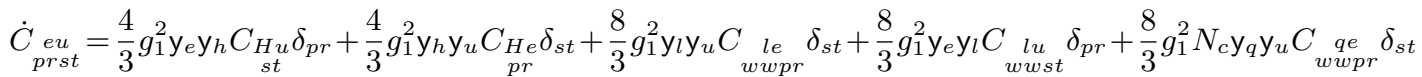

$+\frac{8}{3} g_{1}^{2} N_{c} \mathrm{y}_{e} \mathrm{y}_{q} C_{\underset{q u s t}{(1)}}^{(1)} \delta_{p r}+\frac{4}{3} g_{1}^{2} N_{c} \mathrm{y}_{e} \mathrm{y}_{u} C_{\text {stww }}^{u u} \delta_{p r}+\frac{4}{3} g_{1}^{2} N_{c} \mathrm{y}_{e} \mathrm{y}_{u} C_{\text {wwst }}^{u u} \delta_{p r}+\frac{4}{3} g_{1}^{2} \mathrm{y}_{e} \mathrm{y}_{u} C_{\text {sww }}^{u u} \delta_{p r}$

$+\frac{4}{3} g_{1}^{2} \mathrm{y}_{e} \mathrm{y}_{u} C_{w t s w}^{u u} \delta_{p r}+\frac{4}{3} g_{1}^{2} \mathrm{y}_{e} \mathrm{y}_{u} C_{p r w w}^{e e} \delta_{s t}+\frac{4}{3} g_{1}^{2} \mathrm{y}_{e} \mathrm{y}_{u} C_{w w p r}^{e e} \delta_{s t}+\frac{4}{3} g_{1}^{2} \mathrm{y}_{e} \mathrm{y}_{u} C_{p w w r}^{e e} \delta_{s t}+\frac{4}{3} g_{1}^{2} \mathrm{y}_{e} \mathrm{y}_{u} C_{w r p w}^{e e} \delta_{s t}$

$+\frac{4}{3} g_{1}^{2} N_{c} \mathrm{y}_{u}^{2} C_{\text {prww }}^{e u} \delta_{s t}+\frac{4}{3} g_{1}^{2} \mathrm{y}_{e}^{2} C_{\text {wwst }}^{e u} \delta_{p r}+\frac{4}{3} g_{1}^{2} N_{c} \mathrm{y}_{d} \mathrm{y}_{e} C_{\substack{u d \\ \text { stww }}}^{(1)} \delta_{p r}+\frac{4}{3} g_{1}^{2} N_{c} \mathrm{y}_{d} \mathrm{y}_{u} C_{\text {ped }} \delta_{s t}+12 \mathrm{y}_{e} \mathrm{y}_{u} g_{1}^{2} C_{\text {peu }}^{e u}$

$\dot{C}_{p r s t}^{e d}=\frac{4}{3} g_{1}^{2} \mathrm{y}_{e} \mathrm{y}_{h} C_{s t}^{H d} \delta_{p r}+\frac{4}{3} g_{1}^{2} \mathrm{y}_{d} \mathrm{y}_{h} C_{p r}^{H e} \delta_{s t}+\frac{4}{3} g_{1}^{2} N_{c} \mathrm{y}_{d} \mathrm{y}_{e} C_{s t w w}^{d d} \delta_{p r}+\frac{4}{3} g_{1}^{2} N_{c} \mathrm{y}_{d} \mathrm{y}_{e} C_{w w s t}^{d d} \delta_{p r}+\frac{4}{3} g_{1}^{2} \mathrm{y}_{d} \mathrm{y}_{e} C_{s w w t}^{d d} \delta_{p r}$

$+\frac{4}{3} g_{1}^{2} \mathrm{y}_{d} \mathrm{y}_{e} C_{w t s w}^{d d} \delta_{p r}+\frac{4}{3} g_{1}^{2} \mathrm{y}_{d} \mathrm{y}_{e} C_{p r w w}^{e e} \delta_{s t}+\frac{4}{3} g_{1}^{2} \mathrm{y}_{d} \mathrm{y}_{e} C_{w w p r}^{e e} \delta_{s t}+\frac{4}{3} g_{1}^{2} \mathrm{y}_{d} \mathrm{y}_{e} C_{p w w r}^{e e} \delta_{s t}+\frac{4}{3} g_{1}^{2} \mathrm{y}_{d} \mathrm{y}_{e} C_{w r p w}^{e e} \delta_{s t}$

$+\frac{8}{3} g_{1}^{2} \mathrm{y}_{e} \mathrm{y}_{l} C_{\text {wwst }}^{l d} \delta_{p r}+\frac{8}{3} g_{1}^{2} \mathrm{y}_{d} \mathrm{y}_{l} C_{w w p r}^{l e} \delta_{s t}+\frac{8}{3} g_{1}^{2} N_{c} \mathrm{y}_{e} \mathrm{y}_{q} C_{\substack{q d \\ \text { wwst }}}^{(1)} \delta_{p r}+\frac{8}{3} g_{1}^{2} N_{c} \mathrm{y}_{d} \mathrm{y}_{q} C_{w w p r}^{q e} \delta_{s t}$

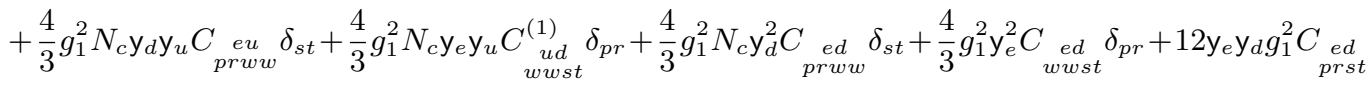

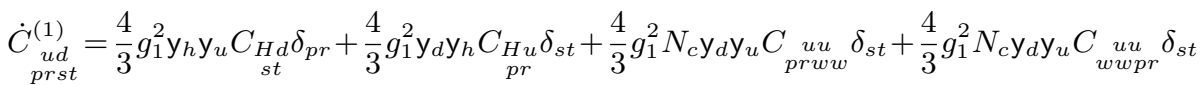

$+\frac{4}{3} g_{1}^{2} \mathbf{y}_{d} \mathbf{y}_{u} C_{p w w r}^{u u} \delta_{s t}+\frac{4}{3} g_{1}^{2} \mathbf{y}_{d} \mathbf{y}_{u} C_{\text {wrpw }}^{u u} \delta_{s t}+\frac{4}{3} g_{1}^{2} N_{c} \mathbf{y}_{d} \mathbf{y}_{u} C_{s t w w}^{d d} \delta_{p r}+\frac{4}{3} g_{1}^{2} N_{c} \mathbf{y}_{d} \mathbf{y}_{u} C_{\text {wwst }}^{d d} \delta_{p r}$

$+\frac{4}{3} g_{1}^{2} \mathrm{y}_{d} \mathrm{y}_{u} C_{\text {swwt }}^{d d} \delta_{p r}+\frac{4}{3} g_{1}^{2} \mathrm{y}_{d} \mathrm{y}_{u} C_{w t s w}^{d d} \delta_{p r}+\frac{8}{3} g_{1}^{2} N_{c} \mathrm{y}_{d} \mathrm{y}_{q} C_{\underset{w w p r}{(1)}}^{(1)} \delta_{s t}+\frac{8}{3} g_{1}^{2} N_{c} \mathrm{y}_{q} \mathrm{y}_{u} C_{\underset{w w s t}{(1)}}^{(1)} \delta_{p r}$

$+\frac{8}{3} g_{1}^{2} \mathrm{y}_{d} \mathrm{y}_{l} C_{\text {wwpr }}^{l u} \delta_{s t}+\frac{8}{3} g_{1}^{2} \mathrm{y}_{l} \mathrm{y}_{u} C_{\text {wwst }}^{l d} \delta_{p r}+\frac{4}{3} g_{1}^{2} N_{c} \mathrm{y}_{d}^{2} C_{\substack{u d \\ \text { prww }}}^{(1)} \delta_{s t}+\frac{4}{3} g_{1}^{2} N_{c} \mathrm{y}_{u}^{2} C_{\substack{u d \\ \text { wwst }}}^{(1)} \delta_{p r}$

$+\frac{4}{3} g_{1}^{2} \mathrm{y}_{d} \mathrm{y}_{e} C_{w w p r}^{e u} \delta_{s t}+\frac{4}{3} g_{1}^{2} \mathrm{y}_{e} \mathrm{y}_{u} C_{\text {wwst }}^{\text {eed }} \delta_{p r}+12 \mathrm{y}_{u} \mathrm{y}_{d} g_{1}^{2} C_{\substack{u d \\ \text { prst }}}^{(1)}+3\left(\frac{N_{c}^{2}-1}{N_{c}^{2}}\right) g_{3}^{2} C_{\substack{u d \\ \text { prst }}}^{(8)}$

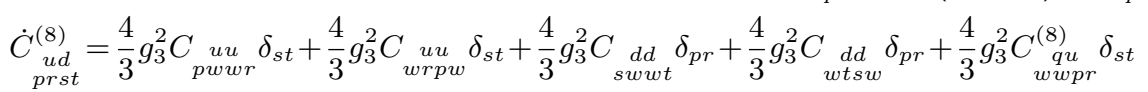

$+\frac{4}{3} g_{3}^{2} C_{\substack{q d \\ w w s t}}^{(8)} \delta_{p r}+\frac{2}{3} g_{3}^{2} C_{\substack{u d \\ \text { prww }}}^{(8)} \delta_{s t}+\frac{2}{3} g_{3}^{2} C_{\substack{u d \\ \text { wwst }}}^{(8)} \delta_{p r}+12\left(\mathrm{y}_{u} \mathrm{y}_{d} g_{1}^{2}-\frac{1}{N_{c}} g_{3}^{2}\right) C_{\substack{u d \\ \text { prst }}}^{(8)}+12 g_{3}^{2} C_{\begin{array}{c}u d \\ \text { prst }\end{array}}^{(1)}$ 


\section{C.8.3 ( $\bar{L} L)(\bar{R} R)$}

$\dot{C}_{p r s t}^{l e}=\frac{4}{3} g_{1}^{2} \mathrm{y}_{h} \mathrm{y}_{l} C_{H_{e}} \delta_{p r}+\frac{4}{3} g_{1}^{2} \mathrm{y}_{e} \mathrm{y}_{h} C_{p r}^{(1)} \delta_{s t}+\frac{8}{3} g_{1}^{2} \mathrm{y}_{e} \mathrm{y}_{l} C_{p r w w}^{l l} \delta_{s t}+\frac{8}{3} g_{1}^{2} \mathrm{y}_{e} \mathrm{y}_{l} C_{w w p r}^{l l} \delta_{s t}+\frac{4}{3} g_{1}^{2} \mathrm{y}_{e} \mathrm{y}_{l} C_{p w w r}^{l l} \delta_{s t}$ $+\frac{4}{3} g_{1}^{2} \mathrm{y}_{e} \mathrm{y}_{l} C_{w r p w}^{l l} \delta_{s t}+\frac{8}{3} g_{1}^{2} N_{c} \mathrm{y}_{e} \mathrm{y}_{q} C_{p r w w}^{(1)} \delta_{s t}+\frac{8}{3} g_{1}^{2} N_{c} \mathrm{y}_{l} \mathrm{y}_{q} C_{w w s t}^{q e} \delta_{p r}+\frac{4}{3} g_{1}^{2} \mathrm{y}_{e}^{2} C_{p r w w}^{l e} \delta_{s t}+\frac{8}{3} g_{1}^{2} \mathrm{y}_{l}^{2} C_{w w s t}^{l e} \delta_{p r}$ $+\frac{4}{3} g_{1}^{2} N_{c} \mathrm{y}_{e} \mathrm{y}_{u} C_{\text {prww }}^{l u} \delta_{s t}+\frac{4}{3} g_{1}^{2} N_{c} \mathrm{y}_{d} \mathrm{y}_{e} C_{p r w w}^{l d} \delta_{s t}+\frac{4}{3} g_{1}^{2} N_{c} \mathrm{y}_{l} \mathrm{y}_{u} C_{\text {stww }}^{e u} \delta_{p r}+\frac{4}{3} g_{1}^{2} N_{c} \mathrm{y}_{d} \mathrm{y}_{l} C_{\text {stww }}^{e d} \delta_{p r}$ $+\frac{4}{3} g_{1}^{2} \mathrm{y}_{e} \mathrm{y}_{l} C_{\text {stww }}^{e e} \delta_{p r}+\frac{4}{3} g_{1}^{2} \mathrm{y}_{e} \mathrm{y}_{l} C_{\text {swwt }}^{e e} \delta_{p r}+\frac{4}{3} g_{1}^{2} \mathrm{y}_{e} \mathrm{y}_{l} C_{w t s w}^{e e} \delta_{p r}+\frac{4}{3} g_{1}^{2} \mathrm{y}_{e} \mathrm{y}_{l} C_{w w s t}^{e e} \delta_{p r}-12 \mathrm{y}_{l} \mathrm{y}_{e} g_{1}^{2} C_{\text {prst }}^{l e}$ $\dot{C}_{p r s t}^{l u}=\frac{4}{3} g_{1}^{2} \mathrm{y}_{h} \mathrm{y}_{l} C_{H_{s t}} \delta_{p r}+\frac{4}{3} g_{1}^{2} \mathrm{y}_{h} \mathrm{y}_{u} C_{\text {pr }}^{(1)} \delta_{s t}+\frac{8}{3} g_{1}^{2} \mathrm{y}_{l} \mathrm{y}_{u} C_{p r w w}^{l l} \delta_{s t}+\frac{8}{3} g_{1}^{2} \mathrm{y}_{l} \mathrm{y}_{u} C_{w w p r}^{l l} \delta_{s t}+\frac{4}{3} g_{1}^{2} \mathrm{y}_{l} \mathrm{y}_{u} C_{p w w r}^{l l} \delta_{s t}$ $+\frac{4}{3} g_{1}^{2} \mathrm{y}_{l} \mathrm{y}_{u} C_{\text {wrpw }}^{l l} \delta_{s t}+\frac{8}{3} g_{1}^{2} N_{c} \mathrm{y}_{q} \mathrm{y}_{u} C_{\substack{l q \\ \text { prww }}}^{(1)} \delta_{s t}+\frac{8}{3} g_{1}^{2} N_{c} \mathrm{y}_{l} \mathrm{y}_{q} C_{\text {wwst }}^{(1)} \delta_{p r}+\frac{4}{3} g_{1}^{2} N_{c} \mathrm{y}_{u}^{2} C_{p r w w}^{l u} \delta_{s t}+\frac{8}{3} g_{1}^{2} \mathrm{y}_{l}^{2} C_{w w s t}^{l u} \delta_{p r}$ $+\frac{4}{3} g_{1}^{2} N_{c} \mathrm{y}_{d} \mathrm{y}_{u} C_{\text {prww }}^{l d} \delta_{s t}+\frac{4}{3} g_{1}^{2} \mathrm{y}_{e} \mathrm{y}_{u} C_{\text {prww }}^{l e} \delta_{s t}+\frac{4}{3} g_{1}^{2} N_{c} \mathrm{y}_{d} \mathrm{y}_{l} C_{\substack{u d \\ \text { stww }}}^{(1)} \delta_{p r}+\frac{4}{3} g_{1}^{2} \mathrm{y}_{e} \mathrm{y}_{l} C_{w w s t}^{e u} \delta_{p r}$ $+\frac{4}{3} g_{1}^{2} N_{c} \mathrm{y}_{l} \mathrm{y}_{u} C_{\text {stww }}^{u u} \delta_{p r}+\frac{4}{3} g_{1}^{2} N_{c} \mathrm{y}_{l} \mathrm{y}_{u} C_{\text {wwst }}^{u u} \delta_{p r}+\frac{4}{3} g_{1}^{2} \mathrm{y}_{l} \mathrm{y}_{u} C_{\text {sww }}^{u u} \delta_{p r}+\frac{4}{3} g_{1}^{2} \mathrm{y}_{l} \mathrm{y}_{u} C_{w t s w}^{u u} \delta_{p r}-12 \mathrm{y}_{l} \mathrm{y}_{u} g_{1}^{2} C_{\text {prst }}$

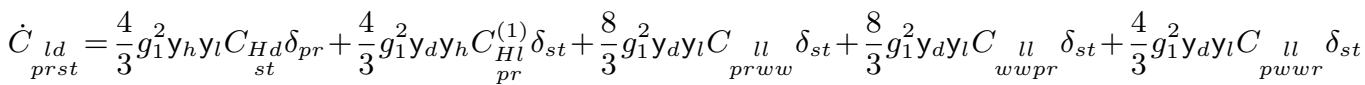
$+\frac{4}{3} g_{1}^{2} \mathbf{y}_{d} \mathbf{y}_{l} C \underset{w r p w}{l l} \delta_{s t}+\frac{8}{3} g_{1}^{2} N_{c} \mathbf{y}_{d} \mathbf{y}_{q} C_{\substack{l q \\ \text { prww }}}^{(1)} \delta_{s t}+\frac{8}{3} g_{1}^{2} N_{c} \mathbf{y}_{l} \mathbf{y}_{q} C_{\substack{q d \\ w w t}}^{(1)} \delta_{p r}+\frac{4}{3} g_{1}^{2} N_{c} \mathrm{y}_{d}^{2} C_{p r w w}^{l d} \delta_{s t}+\frac{8}{3} g_{1}^{2} \mathrm{y}_{l}^{2} C_{w w s t}^{l d} \delta_{p r}$

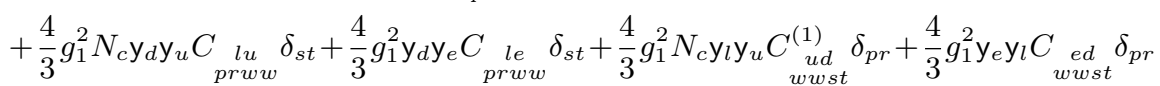
$+\frac{4}{3} g_{1}^{2} N_{c} \mathrm{y}_{d} \mathrm{y}_{l} C_{\text {stww }}^{d d} \delta_{p r}+\frac{4}{3} g_{1}^{2} N_{c} \mathrm{y}_{d} \mathrm{y}_{l} C_{w w s t}^{d d} \delta_{p r}+\frac{4}{3} g_{1}^{2} \mathrm{y}_{d} \mathrm{y}_{l} C_{\text {swwt }}^{d d} \delta_{p r}+\frac{4}{3} g_{1}^{2} \mathrm{y}_{d} \mathrm{y}_{l} C_{w t s w}^{d d} \delta_{p r}-12 \mathrm{y}_{l} \mathrm{y}_{d} g_{1}^{2} C_{p r s t}^{l d}$

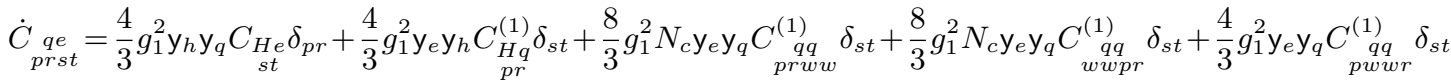
$+\frac{4}{3} g_{1}^{2} \mathrm{y}_{e} \mathrm{y}_{q} C_{w r p w}^{(1)} \delta_{s t}+4 g_{1}^{2} \mathrm{y}_{e} \mathrm{y}_{q} C_{p w w r}^{(3)} \delta_{s t}^{q q}+4 g_{1}^{2} \mathrm{y}_{e} \mathrm{y}_{q} C_{w r p w}^{(3)} \delta_{s t}^{q q}+\frac{8}{3} g_{1}^{2} \mathrm{y}_{e} \mathrm{y}_{l} C_{w w p r}^{(1)} \delta_{w t} \delta_{s t}+\frac{8}{3} g_{1}^{2} \mathrm{y}_{l} \mathrm{y}_{q} C_{w w s t}^{l e} \delta_{p r}$ $+\frac{4}{3} g_{1}^{2} \mathrm{y}_{e}^{2} C_{p r w w}^{q e} \delta_{s t}+\frac{8}{3} g_{1}^{2} N_{c} \mathrm{y}_{q}^{2} C_{w w s t}^{q e} \delta_{p r}+\frac{4}{3} g_{1}^{2} N_{c} \mathrm{y}_{e} \mathrm{y}_{u} C_{p r w w}^{(1)} \delta_{s t}+\frac{4}{3} g_{1}^{2} N_{c} \mathrm{y}_{d} \mathrm{y}_{e} C_{\substack{q d \\ p r w w}}^{(1)} \delta_{s t}$ $+\frac{4}{3} g_{1}^{2} N_{c} \mathrm{y}_{q} \mathrm{y}_{u} C_{\text {stww }}^{e u} \delta_{p r}+\frac{4}{3} g_{1}^{2} N_{c} \mathrm{y}_{d} \mathrm{y}_{q} C_{\text {stww }}^{e d} \delta_{p r}+\frac{4}{3} g_{1}^{2} \mathrm{y}_{e} \mathrm{y}_{q} C_{\text {stww }}^{e e} \delta_{p r}+\frac{4}{3} g_{1}^{2} \mathrm{y}_{e} \mathrm{y}_{q} C_{\text {wwst }}^{e e} \delta_{p r}$ $+\frac{4}{3} g_{1}^{2} \mathrm{y}_{e} \mathrm{y}_{q} C_{s w w t}^{e e} \delta_{p r}+\frac{4}{3} g_{1}^{2} \mathrm{y}_{e} \mathrm{y}_{q} C_{w t s w}^{e e} \delta_{p r}-12 \mathrm{y}_{q} \mathrm{y}_{e} g_{1}^{2} C_{p r s t}^{q e}$

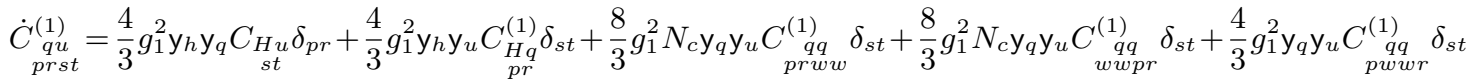
$+\frac{4}{3} g_{1}^{2} \mathrm{y}_{q} \mathrm{y}_{u} C_{\underset{w p w}{(1)}}^{(1)} \delta_{s t}+4 g_{1}^{2} \mathrm{y}_{q} \mathrm{y}_{u} C_{\substack{q q \\ \text { ww } w r}}^{(3)} \delta_{s t}+4 g_{1}^{2} \mathrm{y}_{q} \mathrm{y}_{u} C_{w r p w}^{(3)} \delta_{s t}^{(3)}+\frac{8}{3} g_{1}^{2} \mathrm{y}_{l} \mathrm{y}_{u} C_{w w p r}^{(1)} \delta_{s t}+\frac{4}{3} g_{1}^{2} \mathrm{y}_{e} \mathrm{y}_{u} C_{p r w w}^{q e} \delta_{s t}$ $+\frac{4}{3} g_{1}^{2} N_{c} \mathrm{y}_{d} \mathrm{y}_{u} C_{\substack{q d \\ p r w w}}^{(1)} \delta_{s t}+\frac{4}{3} g_{1}^{2} N_{c} \mathrm{y}_{u}^{2} C_{\substack{q u \\ p w w}}^{(1)} \delta_{s t}+\frac{8}{3} g_{1}^{2} N_{c} \mathrm{y}_{q}^{2} C_{w w s t}^{(1)} \delta_{p r}+\frac{8}{3} g_{1}^{2} \mathrm{y}_{l} \mathrm{y}_{q} C_{w w s t}^{l u} \delta_{p r}+\frac{4}{3} g_{1}^{2} \mathrm{y}_{e} \mathrm{y}_{q} C_{w w s t}^{e u} \delta_{p r}$ $+\frac{4}{3} g_{1}^{2} N_{c} \mathrm{y}_{d} \mathrm{y}_{q} C_{\substack{u d \\ \text { stww }}}^{(1)} \delta_{p r}+\frac{4}{3} g_{1}^{2} N_{c} \mathrm{y}_{q} \mathrm{y}_{u} C_{\text {stww }}^{u u} \delta_{p r}+\frac{4}{3} g_{1}^{2} N_{c} \mathrm{y}_{q} \mathrm{y}_{u} C_{w w s t}^{u u} \delta_{p r}+\frac{4}{3} g_{1}^{2} \mathrm{y}_{q} \mathrm{y}_{u} C_{\text {sww }}^{u u} \delta_{p r}$ $+\frac{4}{3} g_{1}^{2} \mathrm{y}_{q} \mathrm{y}_{u} C_{w t s w}^{u u} \delta_{p r}-12 \mathrm{y}_{q} \mathrm{y}_{u} g_{1}^{2} C_{p r s t}^{(1)}-3\left(\frac{N_{c}^{2}-1}{N_{c}^{2}}\right) g_{3}^{2} C_{p r s t}^{(8)}$ $\underset{\text { Crst }}{\dot{C}_{q u}^{(8)}}=\frac{4}{3} g_{3}^{2} C_{\substack{q q \\ \text { pwr }}}^{(1)} \delta_{s t}+\frac{4}{3} g_{3}^{2} C_{w r p w}^{(1)} \delta_{\text {st }}+4 g_{3}^{2} C_{\text {pwwr }}^{(3)} \delta_{s t}+4 g_{3}^{2} C_{w r p w}^{(3)} \delta_{s t}$ $+\frac{2}{3} g_{3}^{2} C_{p r w w}^{(8)} \delta_{s t}+\frac{2}{3} g_{3}^{2} C_{\substack{q d \\ \text { prww }}}^{(8)} \delta_{s t}+\frac{4}{3} g_{3}^{2} C_{\substack{q u \\ w w s t}}^{(8)} \delta_{p r}+\frac{2}{3} g_{3}^{2} C_{\substack{u d \\ \text { stww }}}^{(8)} \delta_{p r}+\frac{4}{3} g_{3}^{2} C_{\text {sww } u}^{u u} \delta_{p r}+\frac{4}{3} g_{3}^{2} C_{w t s w}^{u u} \delta_{p r}$ $-\left(12 \mathrm{y}_{q} \mathrm{y}_{u} g_{1}^{2}+6\left(N_{c}-\frac{2}{N_{c}}\right) g_{3}^{2}\right) C_{p r s t}^{(8)}-12 g_{3}^{2} C_{\text {prst }}^{(1)}$ 


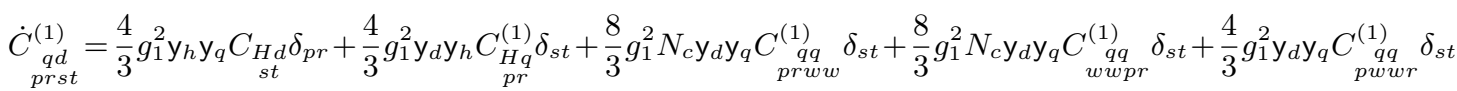

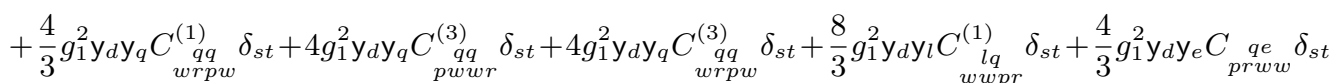
$+\frac{4}{3} g_{1}^{2} N_{c} \mathrm{y}_{d} \mathrm{y}_{u} C_{p r w w}^{(1)} \delta_{s t}+\frac{4}{3} g_{1}^{2} N_{c} \mathrm{y}_{d}^{2} C_{\substack{q d \\ p r w w}}^{(1)} \delta_{s t}+\frac{8}{3} g_{1}^{2} N_{c} y_{q}^{2} C_{\substack{q d \\ \text { wwst }}}^{(1)} \delta_{p r}+\frac{8}{3} g_{1}^{2} \mathrm{y}_{l} \mathrm{y}_{q} C_{\text {wwst }} \delta_{p r}+\frac{4}{3} g_{1}^{2} \mathrm{y}_{e} \mathrm{y}_{q} C_{\substack{\text { ed } \\ w w s t}} \delta_{p r}$

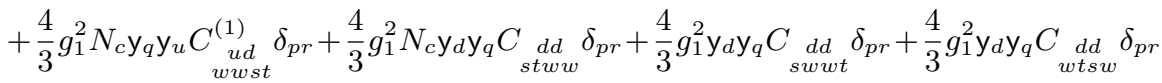
$+\frac{4}{3} g_{1}^{2} N_{c} \mathrm{y}_{d} \mathrm{y}_{q} C_{\text {wwst }}^{d d} \delta_{p r}-12 \mathrm{y}_{q} \mathrm{y}_{d} g_{1}^{2} C_{\substack{q d \\ \text { prst }}}^{(1)}-3\left(\frac{N_{c}^{2}-1}{N_{c}^{2}}\right) g_{3}^{2} C_{\substack{q d \\ \text { prst }}}^{(8)}$

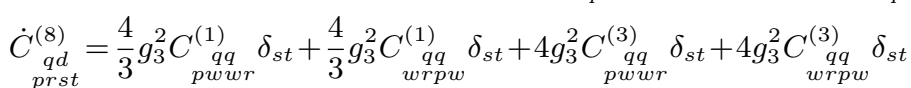

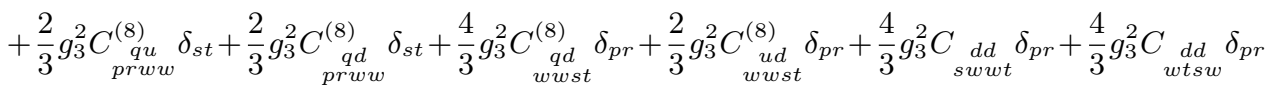
$-\left(12 \mathrm{y}_{q} \mathrm{y}_{d} g_{1}^{2}+6\left(N_{c}-\frac{2}{N_{c}}\right) g_{3}^{2}\right) C_{\substack{q d \\ p r s t}}^{(8)}-12 g_{3}^{2} C_{\substack{q d \\ \text { prst }}}^{(1)}$

\section{C.8.4 $(\bar{L} R)(\bar{R} L)$}

$$
\dot{C}_{\substack{l e d q \\ p r s t}}=-\left(6\left(\mathrm{y}_{d}\left(\mathrm{y}_{q}-\mathrm{y}_{e}\right)+\mathrm{y}_{e}\left(\mathrm{y}_{e}+\mathrm{y}_{q}\right)\right) g_{1}^{2}+3\left(N_{c}-\frac{1}{N_{c}}\right) g_{3}^{2}\right) C_{\substack{l e d q \\ p r s t}}
$$

\section{C.8.5 $(\bar{L} R)(\bar{L} R)$}

$$
\begin{aligned}
& \dot{C}_{\substack{q u q d \\
p t}}^{(1)}=4 g_{1}\left(\mathrm{y}_{q}+\mathrm{y}_{u}\right) C_{d B}\left[Y_{u}^{\dagger}\right]_{p r}-6 g_{2} C_{d W}\left[Y_{u t}^{\dagger}\right]_{p r}-\frac{8}{N_{c}} g_{1}\left(\mathrm{y}_{q}+\mathrm{y}_{u}\right) C_{d B}\left[Y_{u}^{\dagger}\right]_{s r}+\frac{12}{N_{c}} g_{2} C_{d W}\left[Y_{p t}^{\dagger}\right]_{s r} \\
& -8 \frac{N_{c}^{2}-1}{N_{c}^{2}} g_{3} C_{d G}\left[Y_{p t}^{\dagger}\right]_{s r}+4 g_{1}\left(\mathrm{y}_{q}+\mathrm{y}_{d}\right) C_{p r}\left[Y_{d r}^{\dagger}\right]_{s t}-6 g_{2} C_{p r} \underset{p r}{ }\left[Y_{d}^{\dagger}\right]_{s t}-\frac{8}{N_{c}} g_{1}\left(\mathrm{y}_{q}+\mathrm{y}_{d}\right) C_{s r}\left[Y_{d}^{\dagger}\right]_{p t} \\
& +\frac{12}{N_{c}} g_{2} C_{u W}\left[Y_{d}^{\dagger}\right]_{p t}-8 \frac{N_{c}^{2}-1}{N_{c}^{2}} g_{3} C_{u G}\left[Y_{d r}^{\dagger}\right]_{p t} \\
& -\frac{1}{2}\left(\left(3 \mathrm{y}_{d}^{2}+2 \mathrm{y}_{d} \mathrm{y}_{u}+3 \mathrm{y}_{u}^{2}\right) g_{1}^{2}+3 g_{2}^{2}+12\left(N_{c}-\frac{1}{N_{c}}\right) g_{3}^{2}\right) C_{\substack{q u d \\
\text { prst }}}^{(1)} \\
& -\frac{1}{N_{c}}\left(\left(\left(3 \mathrm{y}_{d}^{2}+10 \mathrm{y}_{d} \mathrm{y}_{u}+3 \mathrm{y}_{u}^{2}\right) g_{1}^{2}-3 g_{2}^{2}\right)+8\left(N_{c}-\frac{1}{N_{c}}\right) g_{3}^{2}\right) C_{\substack{q u q d \\
s r p t}}^{(1)} \\
& -\frac{1}{2}\left(1-\frac{1}{N_{c}^{2}}\right)\left(\left(3 \mathrm{y}_{d}^{2}+10 \mathrm{y}_{d} \mathrm{y}_{u}+3 \mathrm{y}_{u}^{2}\right) g_{1}^{2}-3 g_{2}^{2}+4\left(N_{c}-\frac{2}{N_{c}}\right) g_{3}^{2}\right) C_{q u q d}^{(8)} \\
& +2\left(1-\frac{1}{N_{c}^{2}}\right) g_{3}^{2} C_{\substack{q u q d \\
\text { prst }}}^{(8)}
\end{aligned}
$$

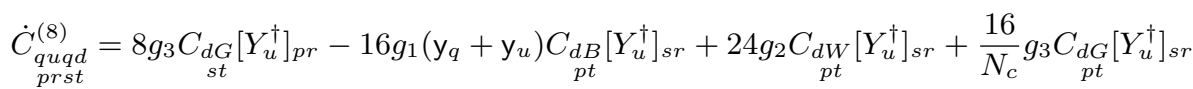

$$
\begin{aligned}
& +8 g_{3} C_{p r}\left[Y_{d}^{\dagger}\right]_{s t}-16 g_{1}\left(\mathrm{y}_{q}+\mathrm{y}_{d}\right) C_{s r}\left[Y_{d}^{\dagger}\right]_{p t}+24 g_{2} C_{u W}\left[Y_{d r}^{\dagger}\right]_{p t}+\frac{16}{N_{c}} g_{3} C_{s G}\left[Y_{d}^{\dagger}\right]_{p t} \\
& +8 g_{3}^{2} C_{\substack{q u q d \\
\text { prst }}}^{(1)}+\left(-2\left(3 \mathrm{y}_{d}^{2}+10 \mathrm{y}_{d} \mathrm{y}_{u}+3 \mathrm{y}_{u}^{2}\right) g_{1}^{2}+6 g_{2}^{2}+16 \frac{1}{N_{c}} g_{3}^{2}\right) C_{\substack{q u q d \\
s r p t}}^{(1)} \\
& +\left(\left(-\frac{3}{2} \mathrm{y}_{d}^{2}-\mathrm{y}_{d} \mathrm{y}_{u}-\frac{3}{2} \mathrm{y}_{u}^{2}\right) g_{1}^{2}-\frac{3}{2} g_{2}^{2}+2\left(N_{c}-\frac{1}{N_{c}}\right) g_{3}^{2}\right) C_{\substack{q u q d \\
p r s t}}^{(8)} \\
& +\frac{1}{N_{c}}\left(\left(3 \mathrm{y}_{d}^{2}+10 \mathrm{y}_{d} \mathrm{y}_{u}+3 \mathrm{y}_{u}^{2}\right) g_{1}^{2}-3 g_{2}^{2}+4\left(-N_{c}-\frac{2}{N_{c}}\right) g_{3}^{2}\right) C_{\substack{q u q d \\
s p t}}^{(8)}
\end{aligned}
$$




$$
\begin{aligned}
& \underset{\substack{l e q u \\
\text { prst }}}{\dot{C}^{(1)}}=-\left(6\left(\mathrm{y}_{e}^{2}+\mathrm{y}_{e}\left(\mathrm{y}_{u}-\mathrm{y}_{q}\right)+\mathrm{y}_{q} \mathrm{y}_{u}\right) g_{1}^{2}+3\left(N_{c}-\frac{1}{N_{c}}\right) g_{3}^{2}\right) C_{\substack{l e q u \\
p r s t}}^{(1)} \\
& -\left(24\left(\mathrm{y}_{q}+\mathrm{y}_{u}\right)\left(2 \mathrm{y}_{e}-\mathrm{y}_{q}+\mathrm{y}_{u}\right) g_{1}^{2}-18 g_{2}^{2}\right) C_{l e q u}^{(3)} \text { prst } \\
& \underset{p r s t}{\dot{C}_{l e q u}^{(3)}}=g_{1}\left(\mathrm{y}_{q}+\mathrm{y}_{u}\right) C_{p r}\left[Y_{u}^{\dagger}\right]_{s t}-\frac{3}{2} g_{2} C_{d W}\left[Y_{u}^{\dagger}\right]_{p r}+g_{1}\left(\mathrm{y}_{l}+\mathrm{y}_{e}\right) C_{s t}\left[Y_{e}^{\dagger}\right]_{p r}-\frac{3}{2} g_{2} C_{e W}\left[Y_{e}^{\dagger}\right]_{s t} \\
& +\left(\left(2\left(\mathrm{y}_{e}^{2}-\mathrm{y}_{e} \mathrm{y}_{q}+\mathrm{y}_{e} \mathrm{y}_{u}-2 \mathrm{y}_{q}^{2}+5 \mathrm{y}_{q} \mathrm{y}_{u}-2 \mathrm{y}_{u}^{2}\right) g_{1}^{2}-3 g_{2}^{2}\right)+\left(N_{c}-\frac{1}{N_{c}}\right) g_{3}^{2}\right) C_{\substack{l e q u \\
\text { prst }}}^{(3)} \\
& +\frac{1}{8}\left(-4\left(\mathrm{y}_{q}+\mathrm{y}_{u}\right)\left(2 \mathrm{y}_{e}-\mathrm{y}_{q}+\mathrm{y}_{u}\right) g_{1}^{2}+3 g_{2}^{2}\right) C_{l e q u}^{(1)} \text { prst }
\end{aligned}
$$

Open Access. This article is distributed under the terms of the Creative Commons Attribution License (CC-BY 4.0), which permits any use, distribution and reproduction in any medium, provided the original author(s) and source are credited.

\section{References}

[1] ATLAS collaboration, Observation of a new particle in the search for the Standard Model Higgs boson with the ATLAS detector at the LHC, Phys. Lett. B 716 (2012) 1 [arXiv:1207.7214] [INSPIRE].

[2] CMS collaboration, Observation of a new boson at a mass of $125 \mathrm{GeV}$ with the CMS experiment at the LHC, Phys. Lett. B $\mathbf{7 1 6}$ (2012) 30 [arXiv:1207.7235] [INSPIRE].

[3] B. Grinstein and M. Trott, A Higgs-Higgs bound state due to new physics at a TeV, Phys. Rev. D 76 (2007) 073002 [arXiv:0704.1505] [InSPIRE].

[4] R. Contino, C. Grojean, M. Moretti, F. Piccinini and R. Rattazzi, Strong double Higgs production at the LHC, JHEP 05 (2010) 089 [arXiv:1002.1011] [INSPIRE].

[5] R. Alonso, M.B. Gavela, L. Merlo, S. Rigolin and J. Yepes, The effective chiral Lagrangian for a light dynamical "Higgs particle", Phys. Lett. B 722 (2013) 330 [Erratum ibid. B 726 (2013) 926] [arXiv:1212.3305] [INSPIRE].

[6] G. Buchalla, O. Catà and C. Krause, Complete electroweak chiral Lagrangian with a light Higgs at NLO, Nucl. Phys. B 880 (2014) 552 [arXiv:1307.5017] [InSPIRE].

[7] I. Brivio et al., Disentangling a dynamical Higgs, JHEP 03 (2014) 024 [arXiv:1311.1823] [INSPIRE].

[8] W. Buchmüller and D. Wyler, Effective Lagrangian analysis of new interactions and flavor conservation, Nucl. Phys. B 268 (1986) 621 [INSPIRE].

[9] B. Grzadkowski, M. Iskrzynski, M. Misiak and J. Rosiek, Dimension-six terms in the Standard Model Lagrangian, JHEP 10 (2010) 085 [arXiv:1008.4884] [INSPIRE].

[10] E.E. Jenkins, A.V. Manohar and M. Trott, Renormalization group evolution of the Standard Model dimension six operators II: Yukawa dependence, JHEP 01 (2014) 035 [arXiv: 1310.4838] [INSPIRE].

[11] E.E. Jenkins, A.V. Manohar and M. Trott, Naive dimensional analysis counting of gauge theory amplitudes and anomalous dimensions, Phys. Lett. B 726 (2013) 697 [arXiv:1309.0819] [INSPIRE]. 
[12] E.E. Jenkins, A.V. Manohar and M. Trott, Renormalization group evolution of the Standard Model dimension six operators I: formalism and $\lambda$ dependence, JHEP 10 (2013) 087 [arXiv: 1308.2627] [INSPIRE].

[13] C. Grojean, E.E. Jenkins, A.V. Manohar and M. Trott, Renormalization group scaling of Higgs operators and $\Gamma(h \rightarrow \gamma \gamma)$, JHEP 04 (2013) 016 [arXiv:1301.2588] [INSPIRE].

[14] R.S. Chivukula and H. Georgi, Composite technicolor Standard Model, Phys. Lett. B 188 (1987) 99 [INSPIRE].

[15] G. D'Ambrosio, G.F. Giudice, G. Isidori and A. Strumia, Minimal flavor violation: an effective field theory approach, Nucl. Phys. B 645 (2002) 155 [hep-ph/0207036] [INSPIRE].

[16] G.F. Giudice, C. Grojean, A. Pomarol and R. Rattazzi, The strongly-interacting light Higgs, JHEP 06 (2007) 045 [hep-ph/0703164] [INSPIRE].

[17] C.-Y. Chen, S. Dawson and C. Zhang, Electroweak effective operators and Higgs physics, Phys. Rev. D 89 (2014) 015016 [arXiv:1311.3107] [inSPIRE].

[18] A. Manohar and H. Georgi, Chiral quarks and the nonrelativistic quark model, Nucl. Phys. B 234 (1984) 189 [InSPIRE].

[19] J. Elias-Miró, J.R. Espinosa, E. Masso and A. Pomarol, Renormalization of dimension-six operators relevant for the Higgs decays $h \rightarrow \gamma \gamma, \gamma Z$, JHEP 08 (2013) 033 [arXiv:1302.5661] [INSPIRE].

[20] J. Elias-Miro, J.R. Espinosa, E. Masso and A. Pomarol, Higgs windows to new physics through $D=6$ operators: constraints and one-loop anomalous dimensions, JHEP 11 (2013) 066 [arXiv:1308.1879] [INSPIRE].

[21] A. Pomarol, Invisibles seminar: 59 ways to leave the SM and implication for Higgs physics, http://invisibles.eu/journal-club/59-ways-leave-sm-and-implication-higgs-physics, June 25 2013.

[22] C.W. Bauer and A.V. Manohar, Renormalization group scaling of the $1 / m^{2}$ HQET Lagrangian, Phys. Rev. D 57 (1998) 337 [hep-ph/9708306] [INSPIRE].

[23] C. Arzt, M.B. Einhorn and J. Wudka, Patterns of deviation from the Standard Model, Nucl. Phys. B 433 (1995) 41 [hep-ph/9405214] [INSPIRE].

[24] E.E. Jenkins, A.V. Manohar and M. Trott, On gauge invariance and minimal coupling, JHEP 09 (2013) 063 [arXiv:1305.0017] [INSPIRE].

[25] A.Y. Morozov, Matrix of mixing of scalar and vector mesons of dimension $D \leq 8$ in $Q C D$ (in Russian), Sov. J. Nucl. Phys. 40 (1984) 505 [Yad. Fiz. 40 (1984) 788] [inSPIRE].

[26] E. Braaten, C.-S. Li and T.-C. Yuan, The evolution of Weinberg's gluonic CP violation operator, Phys. Rev. Lett. 64 (1990) 1709 [INSPIRE].

[27] G. Boyd, A.K. Gupta, S.P. Trivedi and M.B. Wise, Effective Hamiltonian for the electric dipole moment of the neutron, Phys. Lett. B 241 (1990) 584 [inSPIRE].

[28] K. Hagiwara, S. Ishihara, R. Szalapski and D. Zeppenfeld, Low-energy effects of new interactions in the electroweak boson sector, Phys. Rev. D 48 (1993) 2182 [InSPIRE].

[29] K. Hagiwara, R. Szalapski and D. Zeppenfeld, Anomalous Higgs boson production and decay, Phys. Lett. B 318 (1993) 155 [hep-ph/9308347] [INSPIRE].

[30] S. Alam, S. Dawson and R. Szalapski, Low-energy constraints on new physics revisited, Phys. Rev. D 57 (1998) 1577 [hep-ph/9706542] [INSPIRE]. 
[31] Z. Han and W. Skiba, Effective theory analysis of precision electroweak data, Phys. Rev. D 71 (2005) 075009 [hep-ph/0412166] [InSPIRE].

[32] C. Zhang and F. Maltoni, Top-quark decay into Higgs boson and a light quark at next-to-leading order in QCD, Phys. Rev. D 88 (2013) 054005 [arXiv: 1305.7386] [InSPIRE].

[33] G. Altarelli and L. Maiani, Octet enhancement of nonleptonic weak interactions in asymptotically free gauge theories, Phys. Lett. B 52 (1974) 351 [INSPIRE].

[34] M.A. Shifman, A.I. Vainshtein and V.I. Zakharov, Nonleptonic decays of $K$ mesons and hyperons, Sov. Phys. JETP 45 (1977) 670 [Zh. Eksp. Teor. Fiz. 72 (1977) 1275] [InSPIRE].

[35] F.J. Gilman and M.B. Wise, Effective Hamiltonian for $\Delta s=1$ weak nonleptonic decays in the six quark model, Phys. Rev. D 20 (1979) 2392 [INSPIRE].

[36] E.G. Floratos, D.A. Ross and C.T. Sachrajda, Higher order effects in asymptotically free gauge theories: the anomalous dimensions of Wilson operators, Nucl. Phys. B 129 (1977) 66 [Erratum ibid. B 139 (1978) 545] [INSPIRE].

[37] E.G. Floratos, D.A. Ross and C.T. Sachrajda, Higher order effects in asymptotically free gauge theories. 2. Flavor singlet Wilson operators and coefficient functions, Nucl. Phys. B 152 (1979) 493 [INSPIRE].

[38] F.J. Gilman and M.B. Wise, $K \rightarrow \pi e^{+} e^{-}$in the six quark model, Phys. Rev. D 21 (1980) 3150 [inSPIRE].

[39] F.J. Gilman and M.B. Wise, $K^{0}-\bar{K}^{0}$ mixing in the six quark model, Phys. Rev. D 27 (1983) 1128 [INSPIRE].

[40] J. Bijnens and M.B. Wise, Electromagnetic contribution to $\epsilon^{\prime} / \epsilon$, Phys. Lett. B 137 (1984) 245 [inSPIRE].

[41] B. Grinstein, R.P. Springer and M.B. Wise, Strong interaction effects in weak radiative $\bar{B}$ meson decay, Nucl. Phys. B 339 (1990) 269 [INSPIRE].

[42] W.A. Bardeen, A.J. Buras, D.W. Duke and T. Muta, Deep inelastic scattering beyond the leading order in asymptotically free gauge theories, Phys. Rev. D 18 (1978) 3998 [INSPIRE].

[43] A.J. Buras, M. Jamin and P.H. Weisz, Leading and next-to-leading QCD corrections to $\epsilon$ parameter and $B^{0}-\bar{B}^{0}$ mixing in the presence of a heavy top quark, Nucl. Phys. B 347 (1990) 491 [INSPIRE].

[44] A.J. Buras, M. Jamin, M.E. Lautenbacher and P.H. Weisz, Two loop anomalous dimension matrix for $\Delta S=1$ weak nonleptonic decays. 1. $O\left(\alpha_{s}^{2}\right)$, Nucl. Phys. B 400 (1993) 37 [hep-ph/9211304] [INSPIRE].

[45] G. Buchalla, A.J. Buras and M.K. Harlander, The anatomy of $\epsilon^{\prime} / \epsilon$ in the Standard Model, Nucl. Phys. B 337 (1990) 313 [inSPIRE].

[46] M. Ciuchini, E. Franco, G. Martinelli and L. Reina, The $\Delta S=1$ effective Hamiltonian including next-to-leading order QCD and QED corrections, Nucl. Phys. B 415 (1994) 403 [hep-ph/9304257] [INSPIRE].

[47] C. Arzt, M.B. Einhorn and J. Wudka, Effective Lagrangian approach to precision measurements: the anomalous magnetic moment of the muon, Phys. Rev. D 49 (1994) 1370 [hep-ph/9304206] [INSPIRE].

[48] G. Degrassi, E. Franco, S. Marchetti and L. Silvestrini, QCD corrections to the electric dipole moment of the neutron in the MSSM, JHEP 11 (2005) 044 [hep-ph/0510137] [INSPIRE]. 
[49] J. Brod, U. Haisch and J. Zupan, Constraints on CP-violating Higgs couplings to the third generation, JHEP 11 (2013) 180 [arXiv:1310.1385] [INSPIRE].

[50] J. Gao, C.S. Li and C.P. Yuan, NLO QCD corrections to dijet production via quark contact interactions, JHEP 07 (2012) 037 [arXiv: 1204.4773] [INSPIRE].

[51] F. Borzumati, C. Greub, T. Hurth and D. Wyler, Gluino contribution to radiative B decays: organization of QCD corrections and leading order results, Phys. Rev. D 62 (2000) 075005 [hep-ph/9911245] [INSPIRE].

[52] A.J. Buras, M. Misiak and J. Urban, Two loop QCD anomalous dimensions of flavor changing four quark operators within and beyond the Standard Model, Nucl. Phys. B 586 (2000) 397 [hep-ph/0005183] [INSPIRE].

[53] H. Mebane, N. Greiner, C. Zhang and S. Willenbrock, Effective field theory of precision electroweak physics at one loop, Phys. Lett. B 724 (2013) 259 [arXiv:1304.1789] [INSPIRE].

[54] H. Mebane, N. Greiner, C. Zhang and S. Willenbrock, Constraints on electroweak effective operators at one loop, Phys. Rev. D 88 (2013) 015028 [arXiv:1306.3380] [INSPIRE].

[55] H.D. Politzer, Power corrections at short distances, Nucl. Phys. B 172 (1980) 349 [INSPIRE].

[56] R. Contino, M. Ghezzi, C. Grojean, M. Muhlleitner and M. Spira, Effective Lagrangian for a light Higgs-like scalar, JHEP 07 (2013) 035 [arXiv: 1303.3876] [INSPIRE].

[57] A. Pomarol and F. Riva, Towards the ultimate SM fit to close in on Higgs physics, JHEP 01 (2014) 151 [arXiv:1308.2803] [INSPIRE].

[58] C. Grojean, W. Skiba and J. Terning, Disguising the oblique parameters, Phys. Rev. D 73 (2006) 075008 [hep-ph/0602154] [INSPIRE].

[59] T. Feldmann, M. Jung and T. Mannel, Sequential flavour symmetry breaking, Phys. Rev. D 80 (2009) 033003 [arXiv:0906.1523] [inSPIRE].

[60] E.E. Jenkins and A.V. Manohar, Algebraic structure of lepton and quark flavor invariants and CP-violation, JHEP 10 (2009) 094 [arXiv:0907.4763] [INSPIRE].

[61] R. Alonso, M.B. Gavela, L. Merlo and S. Rigolin, On the scalar potential of minimal flavour violation, JHEP 07 (2011) 012 [arXiv:1103.2915] [INSPIRE].

[62] A.L. Kagan, G. Perez, T. Volansky and J. Zupan, General minimal flavor violation, Phys. Rev. D 80 (2009) 076002 [arXiv:0903.1794] [inSPIRE].

[63] T. Corbett, O.J.P. Eboli, J. Gonzalez-Fraile and M.C. Gonzalez-Garcia, Robust determination of the Higgs couplings: power to the data, Phys. Rev. D 87 (2013) 015022 [arXiv: 1211.4580] [INSPIRE].

[64] L.G. Almeida, S.J. Lee, S. Pokorski and J.D. Wells, Study of the $125 \mathrm{GeV}$ Standard Model Higgs boson partial widths and branching fractions, Phys. Rev. D 89 (2014) 033006 [arXiv: 1311.6721] [INSPIRE].

[65] B. Grinstein and M.B. Wise, Operator analysis for precision electroweak physics, Phys. Lett. B 265 (1991) 326 [inSPIRE].

[66] D.A. Ross and M.J.G. Veltman, Neutral currents in neutrino experiments, Nucl. Phys. B 95 (1975) 135 [inSPIRE].

[67] G. Buchalla, O. Catà and G. D'Ambrosio, Nonstandard Higgs couplings from angular distributions in $h \rightarrow Z \ell^{+} \ell^{-}$, arXiv:1310.2574 [INSPIRE]. 
[68] C.W. Bauer, Z. Ligeti, M. Luke, A.V. Manohar and M. Trott, Global analysis of inclusive B decays, Phys. Rev. D 70 (2004) 094017 [hep-ph/0408002] [INSPIRE].

[69] M.E. Machacek and M.T. Vaughn, Two loop renormalization group equations in a general Quantum Field Theory. 1. Wave function renormalization, Nucl. Phys. B 222 (1983) 83 [INSPIRE].

[70] M.E. Machacek and M.T. Vaughn, Two loop renormalization group equations in a general Quantum Field Theory. 2. Yukawa couplings, Nucl. Phys. B 236 (1984) 221 [inSPIRE].

[71] M.E. Machacek and M.T. Vaughn, Two loop renormalization group equations in a general Quantum Field Theory. 3. Scalar quartic couplings, Nucl. Phys. B 249 (1985) 70 [InSPIRE].

[72] ATLAS collaboration, Search for pair-produced top squarks decaying into a charm quark and the lightest neutralinos with $20.3 \mathrm{fb}^{-1}$ of pp collisions at $\sqrt{s}=8 \mathrm{TeV}$ with the ATLAS detector at the LHC, ATLAS-CONF-2013-068, CERN, Geneva Switzerland (2013).

[73] C. Delaunay, T. Golling, G. Perez and Y. Soreq, Charming the Higgs, arXiv:1310.7029 [INSPIRE].

[74] G. Blankenburg, J. Ellis and G. Isidori, Flavour-changing decays of a $125 \mathrm{GeV}$ Higgs-like particle, Phys. Lett. B 712 (2012) 386 [arXiv:1202.5704] [INSPIRE].

[75] R. Harnik, J. Kopp and J. Zupan, Flavor violating Higgs decays, JHEP 03 (2013) 026 [arXiv:1209.1397] [INSPIRE].

[76] ATLAS collaboration, Combined coupling measurements of the Higgs-like boson with the ATLAS detector using up to $25 \mathrm{fb}^{-1}$ of proton-proton collision data, ATLAS-CONF-2013-034, CERN, Geneva Switzerland (2013).

[77] A.V. Manohar and M.B. Wise, Modifications to the properties of the Higgs boson, Phys. Lett. B 636 (2006) 107 [hep-ph/0601212] [INSPIRE].

[78] L. Bergstrom and G. Hulth, Induced Higgs couplings to neutral bosons in $e^{+} e^{-}$collisions, Nucl. Phys. B 259 (1985) 137 [Erratum ibid. B 276 (1986) 744] [INSPIRE].

[79] ATLAS collaboration, Search for the Standard Model Higgs boson in the $H \rightarrow Z_{\gamma}$ decay mode with pp collisions at $\sqrt{s}=7$ and 8 TeV, ATLAS-CONF-2013-009, CERN, Geneva Switzerland (2013).

[80] CMS collaboration, Search for a Higgs boson decaying into a $Z$ and a photon in pp collisions at $\sqrt{s}=7$ and 8 TeV, Phys. Lett. B 726 (2013) 587 [arXiv:1307.5515] [INSPIRE].

[81] A. Azatov, R. Contino, A. Di Iura and J. Galloway, New prospects for Higgs compositeness in $h \rightarrow Z \gamma$, Phys. Rev. D 88 (2013) 075019 [arXiv:1308.2676] [InSPIRE].

[82] D.C. Kennedy and B.W. Lynn, Electroweak radiative corrections with an effective Lagrangian: four fermion processes, Nucl. Phys. B 322 (1989) 1 [INSPIRE].

[83] M.E. Peskin and T. Takeuchi, Estimation of oblique electroweak corrections, Phys. Rev. D 46 (1992) 381 [inSPIRE].

[84] M. Golden and L. Randall, Radiative corrections to electroweak parameters in technicolor theories, Nucl. Phys. B 361 (1991) 3 [InSPIRE].

[85] B. Holdom and J. Terning, Large corrections to electroweak parameters in technicolor theories, Phys. Lett. B 247 (1990) 88 [INSPIRE].

[86] M. Baak et al., The electroweak fit of the Standard Model after the discovery of a new boson at the LHC, Eur. Phys. J. C 72 (2012) 2205 [arXiv:1209.2716] [INSPIRE]. 
[87] O.J.P. Eboli, J. Gonzalez-Fraile and M.C. Gonzalez-Garcia, Scrutinizing the $Z W^{+} W^{-}$vertex at the Large Hadron Collider at 7 TeV, Phys. Lett. B 692 (2010) 20 [arXiv:1006.3562] [INSPIRE].

[88] T. Corbett, O.J.P. Éboli, J. Gonzalez-Fraile and M.C. Gonzalez-Garcia, Determining triple gauge boson couplings from Higgs data, Phys. Rev. Lett. 111 (2013) 011801 [arXiv: 1304.1151] [INSPIRE].

[89] K. Hagiwara, R.D. Peccei, D. Zeppenfeld and K. Hikasa, Probing the weak boson sector in $e^{+} e^{-} \rightarrow W^{+} W^{-}$, Nucl. Phys. B 282 (1987) 253 [inSPIRE].

[90] K. Hagiwara, S. Ishihara, R. Szalapski and D. Zeppenfeld, Low-energy constraints on electroweak three gauge boson couplings, Phys. Lett. B 283 (1992) 353 [InSPIRE].

[91] G. Isidori, A.V. Manohar and M. Trott, Probing the nature of the Higgs-like boson via $h \rightarrow V \mathcal{F}$ decays, Phys. Lett. B 728 (2014) 131 [arXiv:1305.0663] [InSPIRE].

[92] B. Grinstein, C.W. Murphy and D. Pirtskhalava, Searching for new physics in the three-body decays of the Higgs-like particle, JHEP 10 (2013) 077 [arXiv:1305.6938] [INSPIRE].

[93] A.V. Manohar, An exactly solvable model for dimension six Higgs operators and $h \rightarrow \gamma \gamma$, Phys. Lett. B 726 (2013) 347 [arXiv: 1305.3927] [INSPIRE].

[94] MEG collaboration, J. Adam et al., New constraint on the existence of the $\mu^{+} \rightarrow e^{+} \gamma$ decay, Phys. Rev. Lett. 110 (2013) 201801 [arXiv:1303.0754] [INSPIRE].

[95] ACME collaboration, J. Baron et al., Order of magnitude smaller limit on the electric dipole moment of the electron, Science 343 (2014) 269 [arXiv: 1310.7534] [INSPIRE].

[96] R.F. Dashen, E.E. Jenkins and A.V. Manohar, The $1 / N_{c}$ expansion for baryons, Phys. Rev. D 49 (1994) 4713 [Erratum ibid. D 51 (1995) 2489] [hep-ph/9310379] [INSPIRE].

[97] R.F. Dashen, E.E. Jenkins and A.V. Manohar, Spin flavor structure of large- $N_{c}$ baryons, Phys. Rev. D 51 (1995) 3697 [hep-ph/9411234] [INSPIRE]. 